Der Medizinischen Fakultät der Georg-August-Universität Göttingen eingereicht von Prof. Dr. med. C. Binder

\title{
Investigation of TRAIL-resistance in ovarian cancer cell lines and translational application in primary ovarian cancer cells
}

\author{
INAUGURAL- DISSERTATION \\ zur Erlangung des Doktorgrades \\ der Medizinischen Fakultät \\ der Georg-August-Universität zu Göttingen
}

\author{
vorgelegt von \\ Katharina Haider \\ aus \\ Hamburg \\ Göttingen 2011
}


Diese Dissertation wurde erstellt von Oktober 2009 bis Oktober 2010 am Imperial College London unter der Leitung von Prof. Dr. rer. nat. H. Walczak.

Dekan: Prof. Dr. med. C. Frömmel

I. Berichterstatterin: Prof. Dr. med. C. Binder

II. Berichterstatter/in: Prof. Dr. rer. nat. S. Johnsen

Tag der mündlichen Prüfung: 10.August 2011 


\section{Index}

1. Introduction................................................6

1.1. Cell death.....................................................

1.2. Apoptotic pathways.............................................. 8

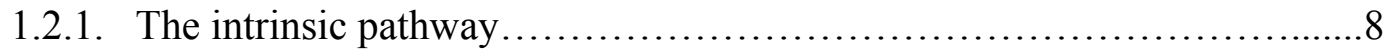

1.2.1.1. Bcl-2 family members .......................................

1.2.2. The extrinsic pathway......................................... 10

1.3. Caspases.......................................................... 11

1.4. TRAIL and TRAIL-receptors...................................... 14

1.4.1. TRAIL and its receptors..................................... 14

1.4.2. TRAIL-induced apoptotic signaling ............................... 15

1.4.3. TRAIL-induced non-apoptotic signaling............................. 17

1.5. Sensitivity versus resistance......................................19

1.6. The role of IAPs in TRAIL-induced apoptosis.........................20

1.7. Physiological role of TRAIL ....................................22

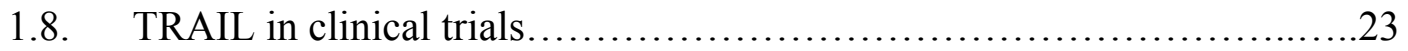

1.9. Combined therapies of TRAIL and small molecule inhibitors..............24

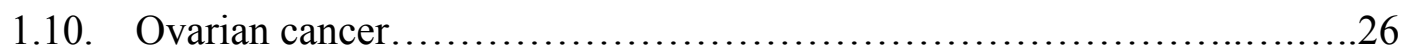

1.10.1. Frequent mutations in different ovarian cancer subtypes................27

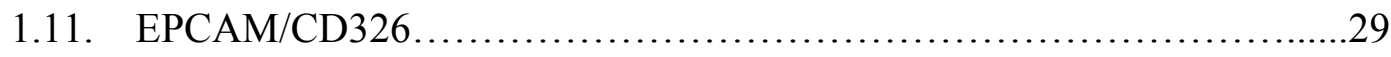

2. Aims and Objectives.......................................31

3. Material and Methods.........................................33

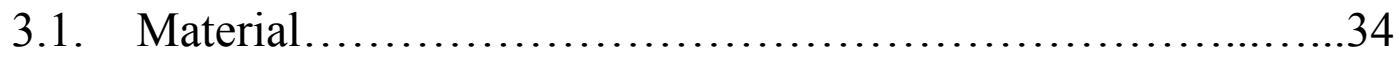

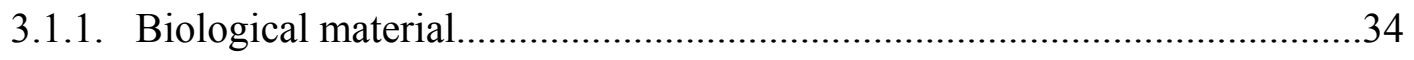

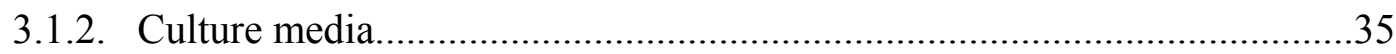

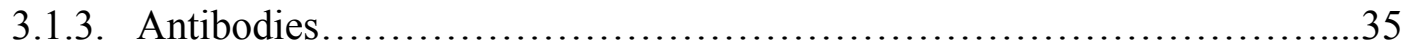

3.1.4. Chemotherapeutics and inhibitors...................................38 


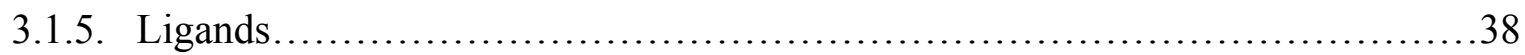

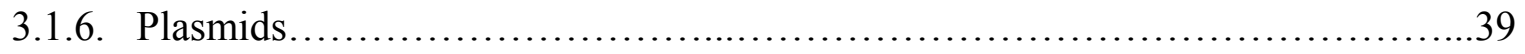

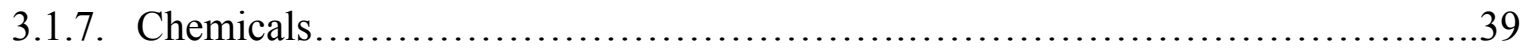

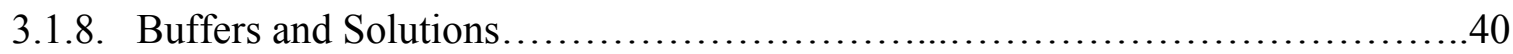

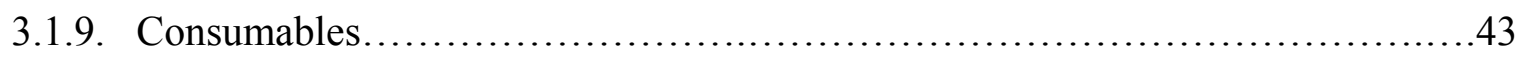

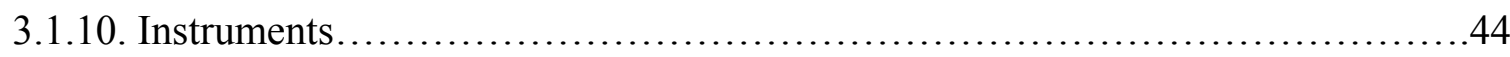

3.2. Methods................................................... 45

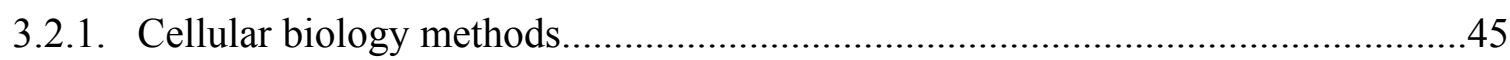

3.2.2. Molecular biology methods..................................................................................4

3.2.3. Biochemical methods....................................................................................49

3.2.4. Isolation of primary ovarian cancer cells from ascitic fluid.........................53

3.2.5. Production of moTAP-TRAIL............................................................................

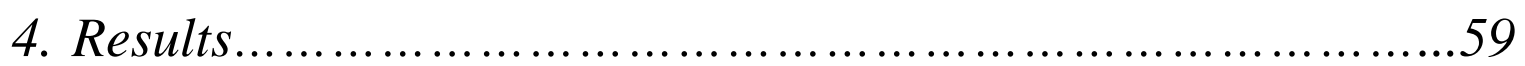

4.1. Assessment of sensitivity of ten different ovarian cancer cell lines to TRAIL-

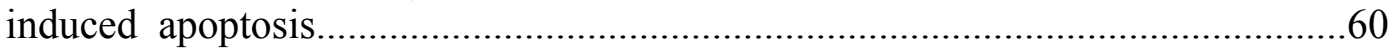

4.2. Toxicity titrations for potential sensitising agents.............................................61

4.3. Bortezomib sensitises KK, PEA-1 and PEA-2 cells but not TOV-112D cells to

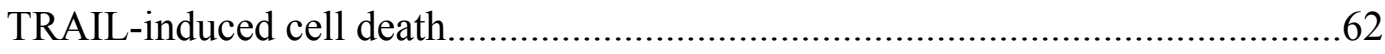

4.4. Bortezomib-mediated sensitisation to TRAIL specifically induced apoptosis.......64

4.5. Smac83 and PIK75 sensitises PEA-1 cells and PEA-2 cells to TRAIL- induced apoptosis

4.6. Cell death induction can be efficiently blocked by caspase-inhibitors but not by necrostatin-1, a RIP-1 kinase activity inhibitor...................................................66

4.7. Molecular changes in the TRAIL-receptor pathway induced by small molecule inhibitors

4.8. Small molecule inhibitors allow for TRAIL-induced cleavage of caspases and cleavage of their substrates. 
4.9. Bortezomib treatment affects the composition of the TRAIL-DISC.....................74

4.10. cIAPs are recruited into the DISC independent of FADD................................75

4.11. Isolation of ovarian cancer cells from ascitic fluid.............................................. 77

4.12. Primary ovarian cancer cells can be sensitised to TRAIL-induced apoptosis by Bortezomib.

4.13. Cisplatin specifically sensitises primary ovarian cancer cells to TRAIL-induced apoptosis.

4.14. Optimisation of the isolation protocol for ovarian cancer cells from ascitic fluid

4.15. EpCAM positive cancer cells but not CD45 positive leucocytes can be sensitised to TRAIL-induced apoptosis via small molecule inhibitors

5. Discussion.

5.1. TRAIL sensitivity does not correlate with histological subtype of ovarian cancer. .88

5.2. Potential sensitising agents exert variable toxicities in ovarian cancer cells lines.

5.3. Implications of Bortezomib-induced sensitisation to TRAIL...............................90

5.4. cIAPs are recruited into the DISC independently of FADD .............................94

5.5. Sensitisation to TRAIL-induced apoptosis by PIK75 or Smac83 ..........................95

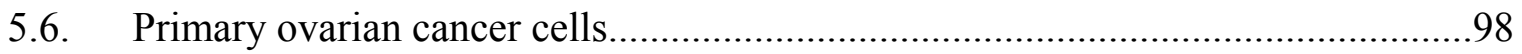

6. Summary and Outlook..............................................................102

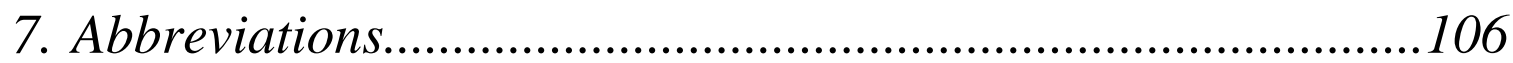

8. References.................................................................................108 
1. Introduction 


\section{Introduction}

\subsection{Cell death}

Mammalian cells die via two major and very distinct forms of cell death - necrosis and apoptosis. In contrast to apoptosis, necrotic cell death almost always has a detrimental effect and is caused by factors such as infection, toxins or trauma (Nicotera et al. 1999). Necrosis usually comprises cell swelling, disruption of the plasma membrane and organelle membranes, leading to an uncontrolled release of cytoplasmic content into the surrounding tissue, typically causing inflammation. Necrotic cell death is a so far thought to be passive event, that does not require a strict regulation and ATP.

Apoptosis, also called programmed cell death, is an organised form of cell death, leading to the unique biochemical and morphological features [figure 1] of apoptosis such as plasma membrane "blebbing", cell shrinkage, chromatin condensation and DNA fragmentation into pieces of 180 base-pair (bp) in length and multiples thereof (Nagata 2000). Early events in apoptosis are detachment of the cell from surrounding cells and from the extracellular matrix. The cell becomes round and smaller and breaks into little pieces called apoptotic bodies, which can then be phagocytosed. Most importantly, the plasma membrane remains intact at any time so that no leakage of intracellular components can occur, thus preventing an immune response resulting in inflammation (Andrade et al. 2010). Biologically, apoptosis plays a crucial role during embryogenesis and controls tissue homeostasis throughout life. Evasion of apoptosis is one of the hallmarks of cancer (Hanahan and Weinberg 2000), but also plays a role in a variety of other human diseases, like certain autoimmune diseases (Prasad and Prabhakar 2003) and metabolic disorders (McKenzie et al. 2004). Apoptosis can be induced via two distinct pathways. The intrinsic and the extrinsic apoptotic pathway.

The extrinsic pathway is induced by extracellular death ligands such as Tumour Necrosis Factor (TNF), CD95L (Apo-1L/FasL) and TNF-related-apoptosis inducing ligand (TRAIL, Apo-2L) binding to their respective death receptors (DRs) on the cell surface (Ashkenazi and Dixit 1998). The intrinsic pathway involves mitochondria and can be induced by DNA-damaging agents, for instance chemotherapy, radiation, UV light, toxins and viral infections (Elmore 2007) 


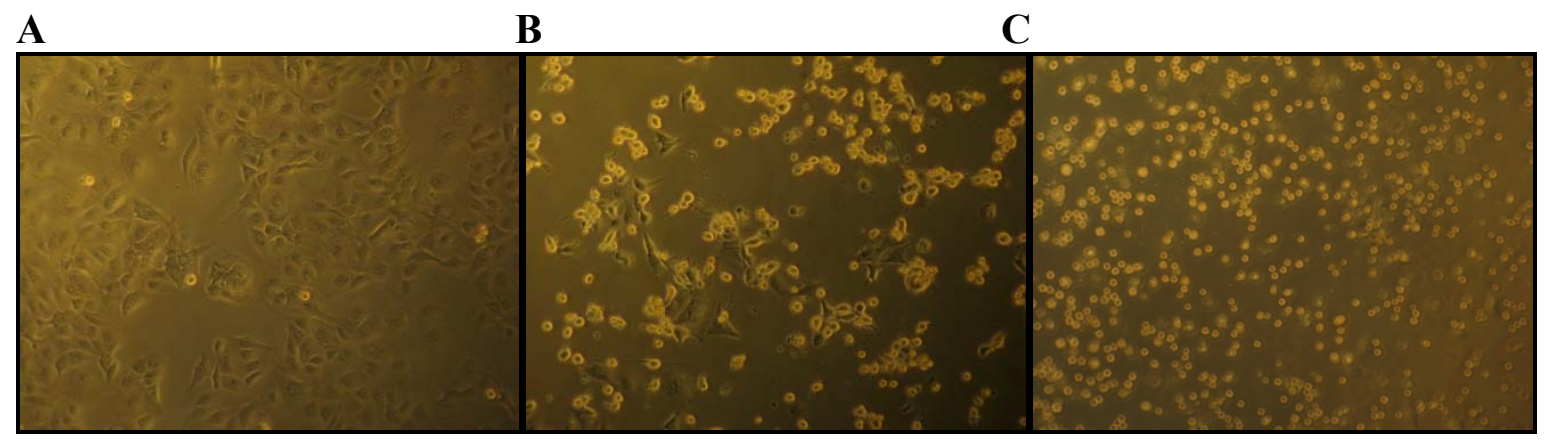

Figure 1: Morphology of apoptotic cells. A. Normal morphology of viable PEO-1 cells. B. PEO-1 cells were treated with 500ng/ml iz-TRAIL for 2 hours which causes characteristic "blebbing" and cell shrinkage. C. After 4h, all cells are small and detached and have broken down into apoptotic bodies.

\subsection{Apoptotic pathways}

\subsubsection{The intrinsic pathway}

The intrinsic pathway can be triggered by a variety of different stimuli like chemotherapy, radiation, toxins, hypoxia, hyperthermia, free radicals but also growth factor deprivation. Intrinsically triggered apoptosis is mainly regulated by proteins of the Bcl-2 (B-celllymphoma 2) family that control the release of pro-apoptotic factors from the mitochondrial intermembrane space into the cytoplasm.

Cellular stress leads to the expression of the pro-apoptotic BH (Bcl-2 homology domains) 3-only proteins like Bik (induced by endoplasmic reticulum stress), Bim or Bad (induced by cytokine deprivation) or Puma and Noxa (induced by DNA damage) (Gonzalvez and Ashkenazi 2010) causing inhibition of the anti-apoptotic proteins Bcl-2 and Bcl-XL, thus allowing oligomerisation and activation of the multi-BH domain proapoptotic proteins Bax and Bak, finally leading to mitochondrial outer-membrane permeabilisation (MOMP) and the release of pro-apoptotic proteins into the cytosol (Waterhouse et al. 2002). The release of cytochrome $\mathrm{C}$ from the mitochondria into the cytosol leads to the assembly of the apoptosome which is composed of cytochrome $\mathrm{C}$, the adaptor protein apoptotic peptidase activating factor 1 (Apaf-1), dATP and serves as an activation platform for procaspase-9. Procaspase-9 contains a caspase recruitment domain (CARD) that is used to mediate specific interactions with the Apaf-1 CARD which becomes exposed on the apoptosome during assembly (Acehan et al. 2002). Along with the apoptosome components that counteract the function of $\mathbf{X}$-linked 
inhibitor of apoptosis protein (XIAP) (Shi 2004) the second mitochondria-derived activator of caspases/ direct IAP binding protein with low isoelectric point (SMAC/DIABLO) is released. XIAP binds to caspase-3,-7 and -9 and inhibits their full activation (Eckelman et al. 2006). However, this effect can be abrogated by the release of SMAC/DIABLO that antagonises the anti-apoptotic effect of XIAP (Verhagen et al. 2000).

\subsubsection{Bcl-2 family members}

The proteins of the Bcl-2 family play a major role in regulating the intrinsic apoptotic pathway. They can be subdivided into three groups based on their expression of the characteristic $\mathrm{BH}$-domains and their ability to act pro-or anti-apoptotically (Lessene et al. 2008; Youle and Strasser 2008) [figure 2].

In mammalian cells, there are five anti-apoptotic Bcl-2 members known (Bcl-2, Bcl-X1, Bcl-w, Mcl-1, Bcl2A1, Bcl-B), which express all four BH domains (BH1-4). They oppose the pro-apoptotic function of the other Bcl-2 family members. The pro-apoptotic group does not only contain the multi-BH domain members Bax, Bak and Bok which comprise BH domain 1-3 but lack domain $\mathrm{BH} 4$, but also comprises members which only contain the $\mathrm{BH} 3$ domain and are consequently called BH3-only proteins.

The anti-apoptotic members are located in the outer mitochondrial membrane stabilising mitochondrial integrity, thereby preventing the release of pro-apoptotic factors such as cytochrome $\mathrm{C}$ and SMAC/DIABLO.

Pro-apoptotic multi-BH domain members Bax and Bak promote caspase activation. They either directly or indirectly induce the release of pro-apoptotic factors from the mitochondria. BH3-only members such as Bad, Bik, Bid, Bim, Bmf, PUMA/NOXA have a conserved BH3 domain that can bind and regulate the anti-apoptotic $\mathrm{Bcl}-2$ proteins to promote apoptosis. Anti-apoptotic Bcl-2 family members become inactivated by binding to $\mathrm{BH} 3$-only proteins (Strasser 2005), whereas pro-apoptotic proteins like Bak/Bax are activated (Willis et al. 2007). 


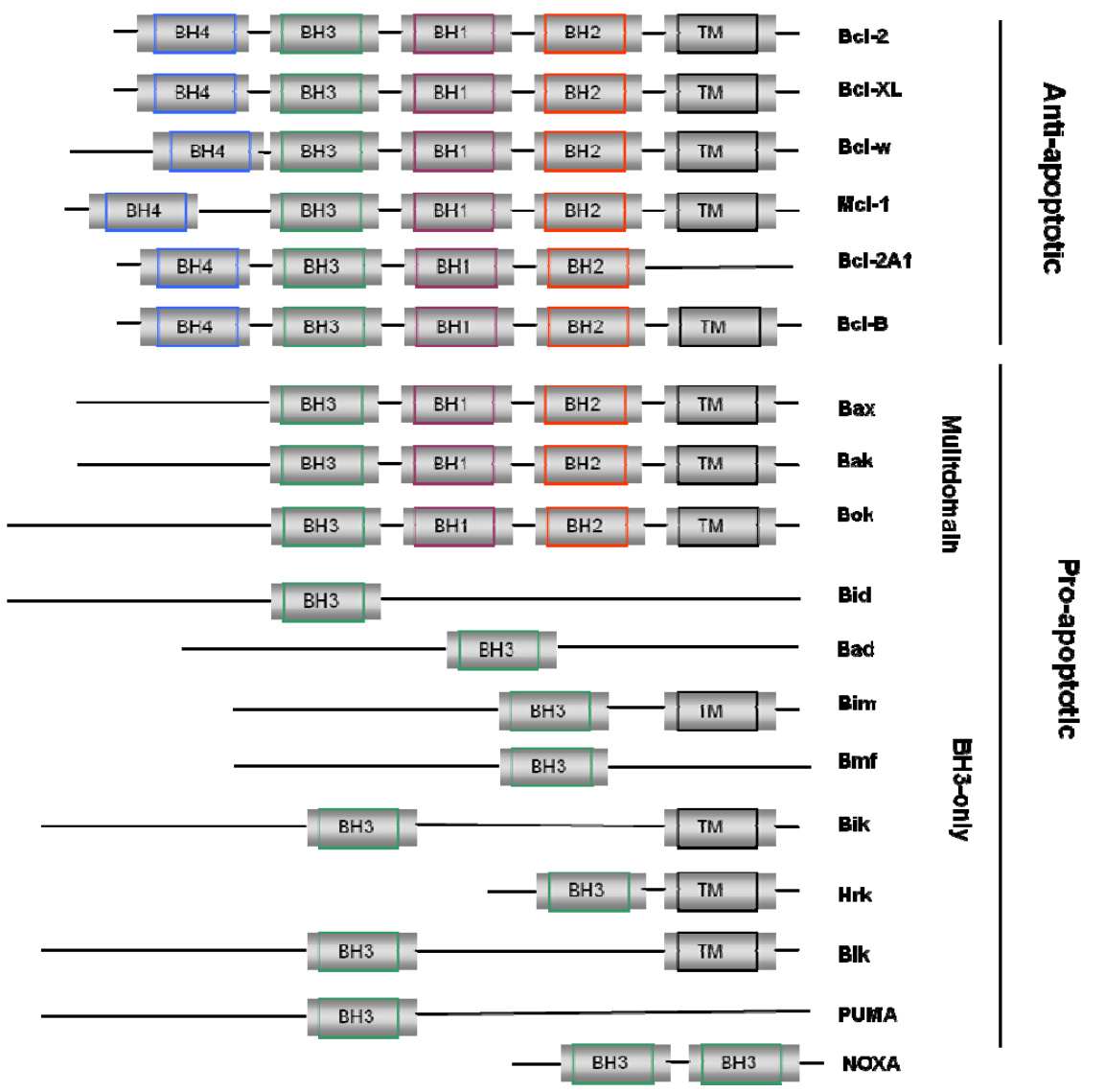

Figure 2: Bcl-2 protein family: Bcl-2 family members comprise one or more Bcl-2 homology (BH)-domains. The anti-apoptotic members comprise all four domains, whereas the pro-apoptotic members either contain BH domain 1-3 or the BH3 domain, only. Most Bcl-2 members additionally contain a transmembrane (TM) domain.

\subsubsection{The extrinsic pathway}

Induction of apoptosis by the extrinsic pathway can involve the activation of six currently known death receptors which exclusively belong to the TNF-R superfamily (Sprick and Walczak 2004). CD95 (Fas, Apo-1), TNFR-1 (p55, CD120a), TRAMP (Apo-3, DR3, WSL-1, LARD) TRAIL-R1 (DR4), TRAIL-R2 (DR5, APO-2, KILLER, TRICK2) and DR6 (TR-7). Death receptors are type I transmembrane proteins and in addition to their cysteine-rich extracellular domains (CRDs) they contain a homologous cytoplasmic sequence of $\sim 80$ amino acids, called the "death domain" (DD) (Ashkenazi and Dixit 1998). Upon binding of their respective trimeric ligand and oligomerisation of the receptors, their DD enables the formation of the death-inducing signalling complex (DISC) which has been described for CD95, TRAIL-R1 and TRAIL-R2. 
Death ligands are type II transmembrane proteins. They can either be displayed on the cell surface or they can be released into the extracellular milieu upon cleavage by proteases (Gonzalvez and Ashkenazi 2010). It is assumed that before ligand binding, TNFR1, TNFR2 but also CD95 and TRAIL-R1 and TRAIL-R2 are preassembled into oligomers via their preligand assembly domain (PLAD) which is located within the CRD of the receptor (Chan 2007). After assembly of the trimeric ligand with the receptors, the adaptor molecule Fas-associated protein with death domain (FADD) is recruited to the receptor via its DD. FADD then recruits the initiator caspases 8 and/or 10 via their death effector domains (DED) to the DISC, and which initiates a caspase cascade by activating the downstream effector caspases 3,6 and 7 .

In some cells, the additional involvement of the mitochondrial pathway is necessary for death receptor-induced apoptosis (Scaffidi et al. 1998). These cells are called type II cells in which in contrast to type I cells, caspase-3 cleavage by caspase- 8 is not sufficient to fully activate caspase- 3 and to induce apoptosis. This was believed to be due to a less efficient DISC formation. The activation of the mitochondrial amplification loop is triggered by caspase-8mediated cleavage of the BH-3 only protein Bcl-2 homology domain3-interacting domain death agonist (Bid) to its active form truncated Bid (tBid). tBid then translocates to the mitochondria membrane, leading to the activation of pro-apoptotic molecules Bax and Bak (Li, H. et al. 1998). However, recently the molecule that discriminates between Type I and Type II has been reported to be XIAP (Jost et al. 2009). Type I cells have been shown to be able to effectively down regulate the levels of XIAP upon a death ligand stimulus, whereas Type II cells can not. They could show that downregulation of XIAP by-passes the requirement of mitochondrial SMAC/DIABLO and sensitises Type II cells to TRAIL-induced apoptosis.

\subsection{Caspases}

Caspases (cysteine-dependent, aspartate-specific acid proteases) are a family of cystein proteases that play an essential role in apoptosis. All caspases are synthesised as catalytically inactive zymogens (protein precursors), and have to undergo an activation process. They can be divided into two groups, the initiator caspases (caspase- $8,-10,-2,-9,-14$ ) and the effector caspases (caspase-3,-6,-7) [figure 3A]. 
Depending on the prodomain, initiator caspases can be further subdivided into CARD-domain containing caspases (caspase 2,-9) or DED-domain containing initiator caspases (caspase-8,10). The activation of DED-containing initiator caspases occurs at the DISC (Caspase-8,-10) (Walczak and Haas 2008), whereas the activation of CARD-domain containing caspases occurs at the apoptosome (caspase-9) or piddosome (caspase-2), respectively (Riedl et al. 2007). Besides the prodomain, initiator caspases contain a large catalytic subunit comprising the active site and a small catalytic subunit.

Effector caspases have a very small prodomain devoid of a CARD or a DED. Otherwise, they only posess the small and large catalytic subunit. Cleavage between the small and large catalytic domains alters their conformation and the caspase is rendered active. Cleaved mature caspases are dimers of catalytic units each containing a large and a small subunit [figure 3B]. In contrast to effector caspases, initiator caspases do not require cleavage to be activated (Stennicke et al. 1999). It has been shown that initiator caspases primarily exist as monomers and lack significant activity in this form (Renatus et al. 2001). For their activation they do not require cleavage but, instead they require to be brought into proximity so that they can build dimers. So, unlike for effector caspases, cleavage is neither required nor sufficient for the activation of initiator caspases.

The hypothesis of the " induced-proximity" claims that the recruitment of initiator caspases to the receptor complex by FADD leads to clustering of initiator caspase zymogens which then leads to self-activation via cross proteolysis. This hypothesis has now been challenged by the model of the "proximity induced dimerisation" which postulates, that initiator caspases exist as inactive monomers and that the primary function of adaptor protein complexes would be to serve as a platform to promote dimerisation (Boatright and Salvesen 2003). 
$\mathbf{A}$

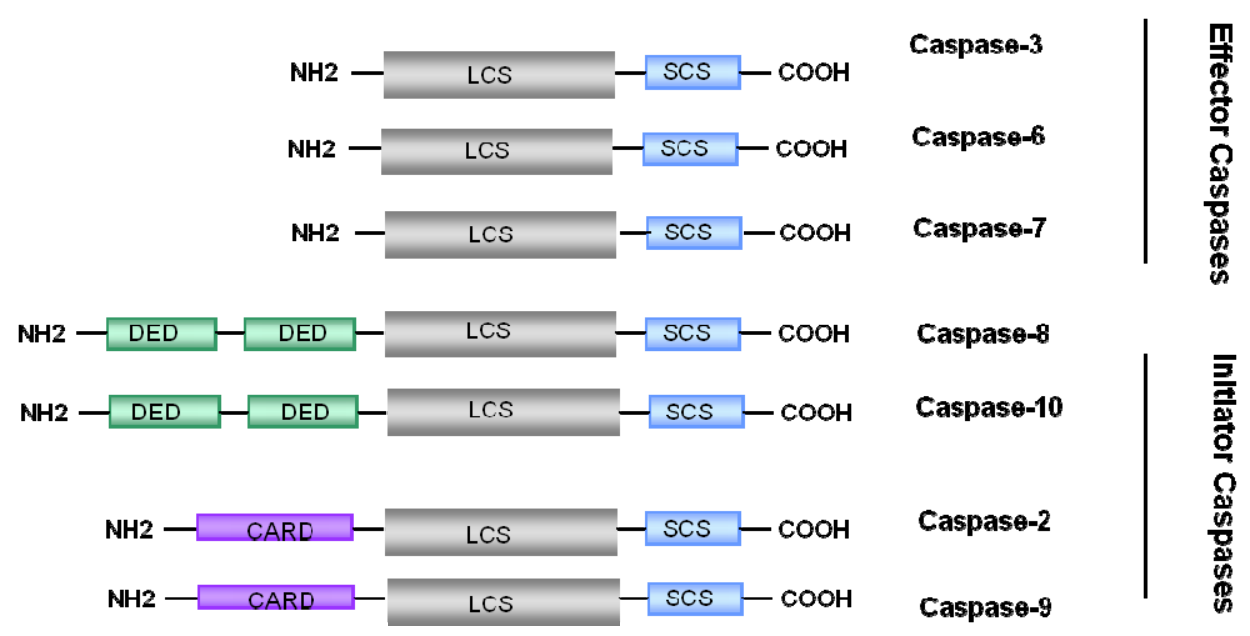

B

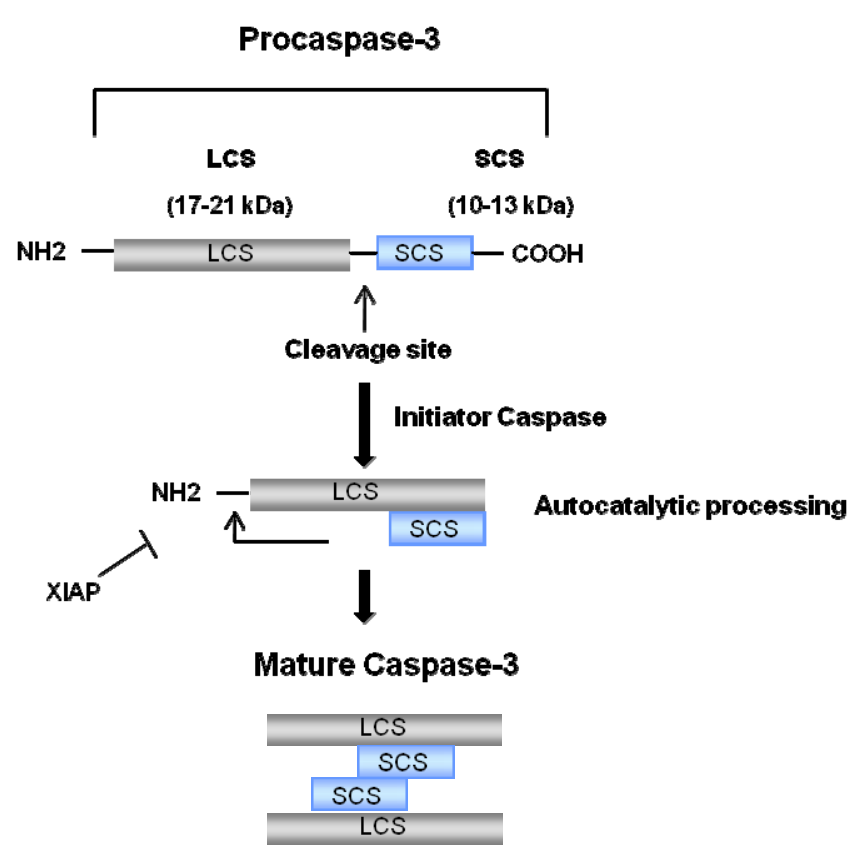

Figure 3: The caspase family: A. According to their function and structure, caspases can be divided into different subgroups. Initiator caspases contain a death effector domain (DED) or a caspase recruitment domain (CARD) domain. Effector caspases contain only a very short prodomain. They also possess the large catalytic subunit (LCS) and the small catalytic subunit (SCS). B. This diagram shows the cleavage and activation of caspase-3. Cleavage at the amino acid position Aspartate 175 leads to autocatalytic processing of caspase-3 in which process the prodomain of caspase-3 is cleaved off at Aspartate 28. XIAP can prevent this cleavage. 


\subsection{TRAIL and TRAIL-Receptors}

\subsubsection{TRAIL and its Receptors}

TRAIL is a type II transmembrane protein which is homologous to CD95L and TNF. It was first cloned and characterised in 1995 (Wiley et al. 1995). TRAIL is expressed by various cells of the immune system, among them natural killer cells (NK cells), T-cells, dendritic cells (DC's) and macrophages. TRAIL can be cleaved from the membrane (mTRAIL) by cysteine proteases (Mariani and Krammer 1998), generating a trimeric, soluble ligand (sTRAIL) (Wajant et al. 2001). Trimeric soluble TRAIL contains a central zinc atom at the trimer interface. The zinc ion is required for maintaining the native structure, stability and biological activity of the ligand in its trimeric structure (Hymowitz et al. 2000). So far, five different receptors for TRAIL have been identified in humans [figure 4]. Only two of them, TRAIL-R1 (Pan et al. 1997) and TRAIL-R2 (Screaton et al. 1997; Walczak et al. 1997) comprise a full length DD and are therefore able to induce apoptosis. TRAIL-R3 (Decoy Receptor (DcR) 1) does not contain an intracellular DD and is anchored to the membrane via a glycosylphosphatidylinositol (GPI) anchor (Degli-Esposti et al. 1997 a). TRAIL-R4 (DcR2) has been described to contain a truncated DD (Pan et al. 1998). TRAIL-R3 and -R4 cannot transmit an apoptotic signal. They have, however, been suggested to act as "decoys" for TRAIL, thereby reducing apoptotic signals by TRAIL-R1 and -R2. It was recently demonstrated that only TRAIL-R3 acts merely as a competitor for TRAIL binding, preventing TRAIL-R1 associated DISC assembly, while TRAIL-R4 interacts with TRAIL-R1 in the native DISC in a TRAIL-dependent manner and prevents TRAIL-R2 co-recruitment to TRAIL-R1 (Merino et al. 2006). However the "decoy" function remains a matter of debate and several studies have challenged its importance for TRAIL signalling (Clancy et al. 2005). Finally, a fifth receptor has been reported for the human system, osteoprotegerin (OPG) a receptor for RANKL (Receptor Activator of NF-кB Ligand) that inhibits osteoclast formation and bone resorption. OPG which only exists in a soluble form (Emery et al. 1998) also binds TRAIL, but with a lower affinity and does not transmit an apoptotic signal. The physiological significance of this interaction remains unclear however, since TRAIL-knockout mice have a normal phenotype and develop normal bone density (Cretney et al. 2002).

In contrast to humans, mice only have one apoptosis-inducing receptor for TRAIL, the murine TRAIL-R (mTRAIL-R) which is highly homologous to both human apoptosis-inducing TRAIL receptors TRAIL-R1 and TRAIL-R2 (Wu et al. 1999). The other murine receptors, 
$\mathrm{mDcR} 1, \mathrm{mDcR} 2 \mathrm{~L}$ and the splice variant $\mathrm{mDcR} 2 \mathrm{~S}$, share a clustered locus. However, functionally, they have not been studied in any particular detail (Schneider et al. 2003).
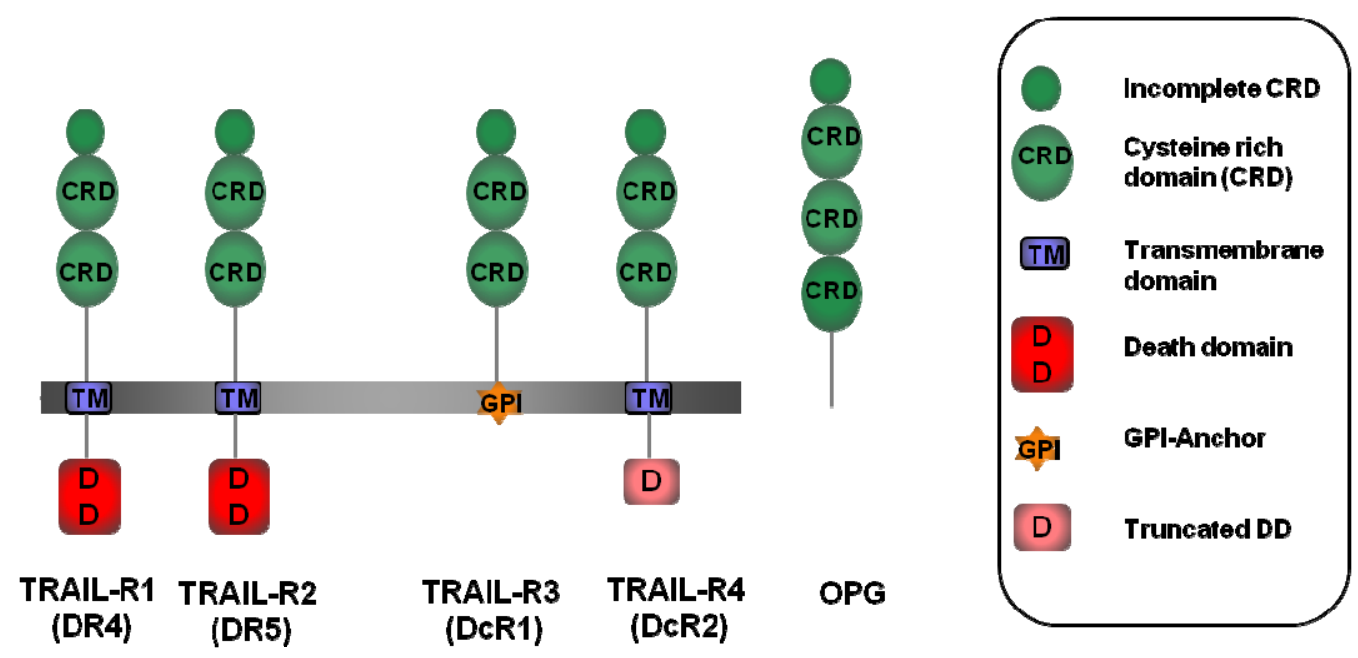

Figure 4: TRAIL-receptor family. TRAIL-R1 and TRAIL-R2 have a functional death domain (DD) and are able to transmit an apoptotic signal. TRAIL-R3 and soluble OPG do not possess a DD at all, whereas TRAIL-R4 comprises a truncated DD.

\subsubsection{TRAIL-induced apoptotic signaling}

As mentioned above, binding of trimeric TRAIL to TRAIL-R1 and TRAIL-R2 leads to homo-or heteromeric trimerisation of the receptors and subsequent formation of the DISC (Walczak and Haas 2008) [figure 5]. Firstly, FADD is recruited to the intracellular DDs of the receptors via its own DD. In addition to the DD, FADD also contains a second functional domain, the death effector domain (DED) by which procaspase- 8 and -10 can be recruited to the DISC, where they are auto-catalytically cleaved and activated. The importance of caspase-10 and the question whether it can substitute for caspase- 8 at the DISC is still controversial. On the one hand it has been reported that caspase-10 transfection did not restore sensitivity of caspase-8-deficient cells to TRAIL-induced apoptosis (Sprick et al. 2002), whereas on the other hand it has been shown that transfection of caspase-10 was able to restore sensitivity (Kischkel et al. 2001; Wang et al. 2001). As a negative regulator of apoptosis, cellular FLICE (caspase-8) -like inhibitory protein (cFLIP) is also recruited to FADD via its DED. cFLIP shares sequence homology with caspase- 8 but lacks enzymatic activity as it contains a mutation in its catalytic subunit. It competes with caspase- 8 for 
binding to the DISC and thereby inhibits activation of caspase-8 (Irmler et al. 1997). cFLIP exists in three different splice variants. $\mathrm{cFLIP}_{\mathrm{L}}, \mathrm{cFLIP}_{\mathrm{S}}$ and $\mathrm{cFLIP}_{\mathrm{R}}$. $\mathrm{cFLIP}_{\mathrm{S}}$ and $\mathrm{cFLIP}_{\mathrm{R}}$ have only been described as potent inhibitors of apoptosis, whereas $\mathrm{CFLIP}_{\mathrm{L}}$ has been described to be able to act in both anti-and also in a pro-apoptotic manner, depending on its expression level. If expressed at a high level, cFLIP $_{\mathrm{L}}$ acts anti-apoptotically by preventing caspase- 8 activation at the DISC. However, when expressed at lower, possibly physiological more relevant levels ( $1 \%$ of caspase- 8 concentration), $\mathrm{cFLIP}_{\mathrm{L}}$ promotes procaspase- 8 activation through hetero-dimerisation (Chang et al. 2002; Boatright et al. 2004; Fricker et al. 2010). Recently it has also been found that the action of $\mathrm{cFLIP}_{\mathrm{L}}$ depends on the strength of the death stimulus (Fricker et al. 2010).

After procaspase- 8 has been activated at the DISC either by heterodimerisation with cFLIP $_{\mathrm{L}}$ or by homo-oligomerisation (Martin et al. 1998; Muzio et al. 1998) it is released into the cytosol, where it can cleave effector caspases such as caspase-3. Caspase-3 cleaves further caspases and proteins, for instance the enzyme poly (ADP-ribose) polymerase (PARP), which was one of the first proteins identified to be a substrate for caspase-3 (Rheaume et al. 1997) In some cells, activation of caspase- 8 at the DISC is not sufficient to fully activate enough caspase-3 to induce apoptosis. These cells rely on the mitochondrial amplification loop induced by cleavage of BH-3-only protein Bid by active caspase- 8 . As mentioned before, the signalling events downstream of the DISC depend very much on whether a particular cell is of "type I" or "type II". 


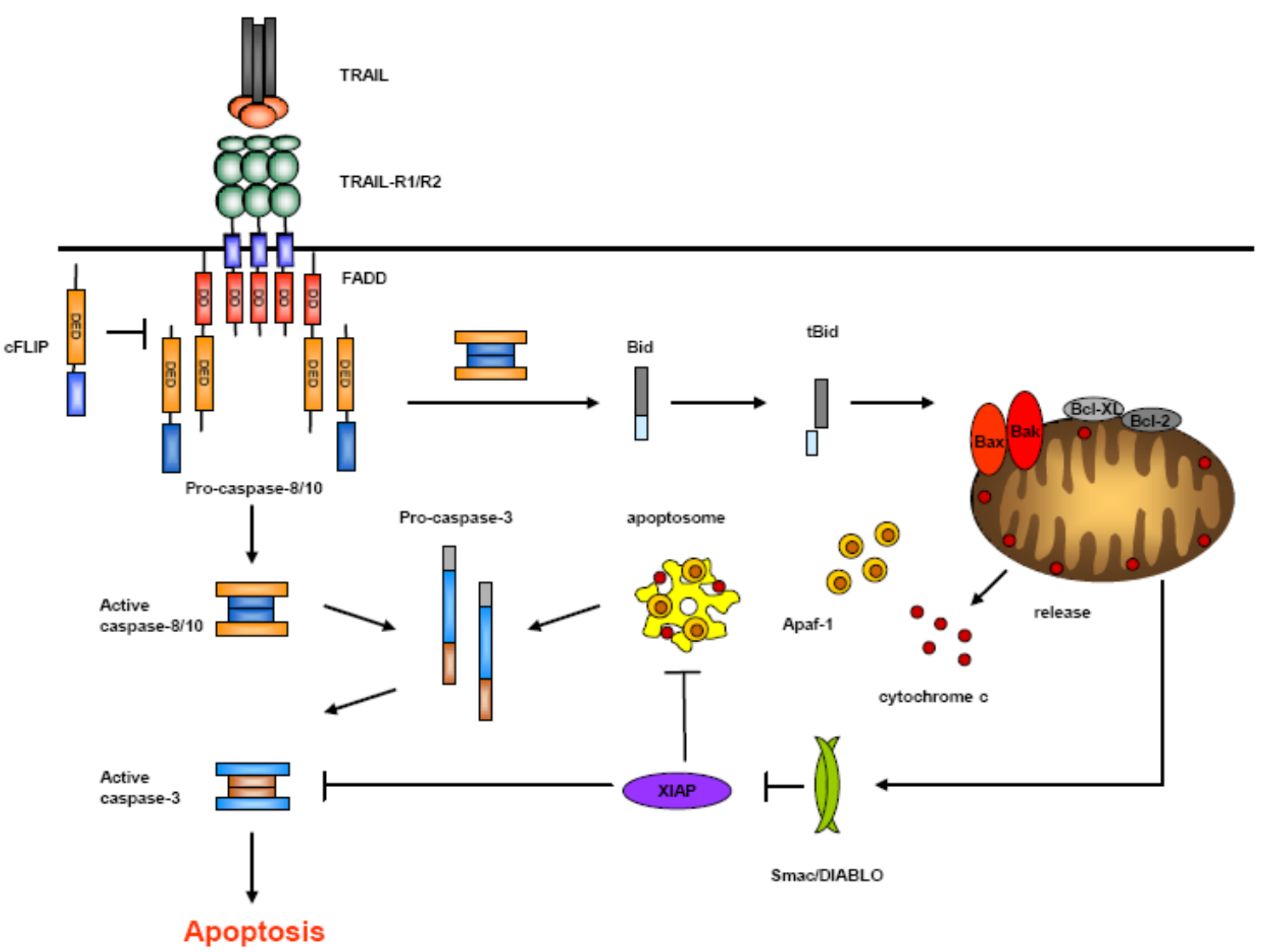

Figure 5: Apoptotic signalling by TRAIL.

Binding of TRAIL to either TRAIL-R1 or -R2 leads to receptor trimerisation and assembly of the deathinducing signalling complex (DISC). FADD is recruited to the DISC via its DD and interacts with procaspase-8 and -10 via the DED. cFLIP contains also contains a DED and can therefore compete with the pro-caspases for binding to FADD. After activation of caspase-8 at the DISC, further downstream caspases are activated, such as caspase-3. Cleavage of Bid to tBid initiates the mitochondrial amplification loop leading to release of cytochrome $\mathrm{c}$ and SMAC/DIABLO from the mitochondria. The apoptosome comprises cytochrome-c, apaf-1 and serves as a activation platform for pro-caspase 9. SMAC/DIABLO counteracts the inhibitory function of XIAP thereby allowing for full activation of caspase-3 and -9 , leading to cell death. Picture from (Cordier et al. 2009, 119).

\subsubsection{TRAIL-induced-non-apoptotic signalling}

Besides induction of apoptosis, TRAIL has also shown to be able to induce non-apoptotic pathways, leading to proliferation, migration and invasion of tumour cells that are resistant to TRAIL-induced apoptosis. This includes the activation of nuclear factor 'kappa-light-chain-enhancer' of activated B-cells (NF-kB) which has been shown to be 
mediated by TRAIL-R1, TRAIL-R2 and also TRAIL-R4 (Degli-Esposti et al. 1997b; MacFarlane 2003).

In the beginning, TRAIL-induced NF- $\kappa \mathrm{B}$ activation was thought to only counteract TRAIL-induced apoptosis. However, in 2006 it was reported that TRAIL-induced tumour cell migration and invasion of apoptosis resistant cholangiocarcinoma cells was dependent on the activation of NF- $\kappa$ B by TRAIL (Ishimura et al. 2006).

Furthermore, the receptor-interacting protein-1 (RIP-1), known to play a role in the TNF-Receptor-complex has been detected in the TRAIL-DISC (Harper et al. 2001) and reported to mediate TRAIL-induced IkB-kinase (IKK) activation (Lin et al. 2000). Additionally, RIP1 has been found to interact with FADD. Although FADD is dispensable for TNF-induced apoptosis, it is essential for the activation of caspase- 8 and NF- $\kappa$ B following TRAIL treatment (Jin and El-Deiry 2006). It was proposed that non-apoptotic signalling by TRAIL was mediated via assembly of a secondary cytosolic signalling complex, which is separated from the DISC. This complex was suggested to contain FADD, caspase-8, TRAF2, RIP1 and IKK- $\gamma$ and to be crucial not only for the activation of NF- $\mathrm{BB}$, but also of the c-Jun-N-terminal kinase ( JNK) and p38 following TRAIL stimulation (Varfolomeev et al. 2005).

Activation of protein kinase $\mathbf{C}$ (PKC) has been reported to inhibit the recruitment of FADD to the TRAIL-DISC, thereby modulating TRAIL sensitivity (Harper et al. 2003)

Furthermore, it was found that Mitogen-activated kinases (MAPKs) affect TRAIL sensitivity (Frese et al. 2003). For caspase-dependent MAPK activation upon TRAIL stimulation mammalian sterile 20-like kinase 1 (Mst1) is required (Song and Lee 2008). It was reported, that caspase-3 and -7 cleave Mst1 at different sites leading to different signalling outcome. Caspase-3 was reported to be an important factor for MAPK and extracellular-signal regulated kinase (ERK) phosphorylation.

The significance of non-apoptotic signalling by TRAIL has also been shown in vivo. Human pancreatic ductal adenocarcinoma cells were orthotopically transplanted in immunodeficient mice (Trauzold et al. 2006). A dramatic increase in metastatic spread upon TRAIL treatment could be observed. This result suggests an explanation for the apparently counterintuitive finding that tumour cells do not lose TRAIL-receptors during tumourigenesis as some can convert the apoptotic signal to a pro-survival or even pro-migratory signal. This highlights the 
importance to find therapy options that not only enhance apoptosis induction but also prevent pro-invasive and pro-migratory TRAIL-signalling.

\subsection{Sensitivity versus resistance}

Resistance to TRAIL-mediated apoptosis of human cancer cell lines is a considerable problem in the treatment of many primary tumours (Koschny et al. 2007a) and can be caused by a great number of different factors along the signalling pathway.

However, many tumours can be sensitised to TRAIL-induced apoptosis by a variety of small-molecule inhibitors (Ganten et al. 2005), chemotherapeutic agents (Zisman et al. 2001), Histone-Deacetylase (HDAC) inhibitors (Dzieran et al. 2008) or $\gamma$-irradiation (Maduro et al. 2008). Very often, however, the exact biochemical mechanism underlying sensitisation remains unknown.

Resistance of tumour cells to TRAIL-induced apoptosis can occur on at least four different levels. These are the receptor level, the DISC, the mitochondria or, further downstream, the level of caspase-3 activation.

High expression of TRAIL-R3 and TRAIL-R4 on cancer cell lines has been proposed to have a decoy function so that such cancer cells would be able to evade killing by TRAIL-mediated apoptosis via TRAIL-R1 and TRAIL-R2 (LeBlanc and Ashkenazi 2003; Kimberley and Screaton 2004). TRAIL-R3 for instance, has been found to be overexpressed in TRAIL-resistant tumours of the gastrointestinal (GI) tract and has been suggested to compete with TRAIL-R1 and TRAIL-R2 for ligand binding, thus having a negative influence on apoptosis (Sheikh et al. 1999). However, as mentioned above, there have been contradicting studies about the influence of decoy receptors on apoptosis. One recent study showed that socalled decoy receptors are even downregulated in prostate cancer while other TRAIL receptors and TRAIL itself are not (Hornstein et al. 2008).

Furthermore, there have been reports on the upregulation of TRAIL-R1 and TRAIL-R2 upon treatment with chemotherapeutic agents (Wu et al. 2000; Ganten et al. 2004).

Although, the upregulation of TRAIL-R1 and -R2 can contribute to TRAIL sensitisation, it is not sufficient to explain the effect. For instance, Ganten et al. (2004) showed that upregulation of TRAIL-R1 and-R2 is not essential for the sensitisation effect they observe with 5-Fluourouracil (5-FU) to TRAIL-induced apoptosis. 
As a direct competitor of caspase- 8 and-10 for binding to FADD, cFLIP has been shown to inhibit TRAIL-induced apoptosis and thereby conveys resistance when expressed at high levels. High cFLIP expression has indeed been correlated with TRAIL resistance in cancer cells (Horak et al. 2005). Furthermore, knock-down of cFLIP restored TRAIL sensitivity in different cancer cell lines, including ovarian cancer cell lines (Siegmund et al. 2002; Ganten et al. 2004; Clarke and Tyler 2007; Geserick et al. 2008). Moreover, epigenetically silenced caspase-8 blocks TRAIL-induced apoptosis at the initiation step (Hopkins-Donaldson et al. 2000).

Another crucial factor causing TRAIL resistance in cancer cells is the overexpression of XIAP, e.g. in pancreatic adenocarcinoma samples. Inhibitor of apoptosis proteins (IAPs), for example XIAP, counteract apoptosis by binding to and inhibiting caspase-3, -7, and -9 (Micheau and Merino 2004; Shi 2004). SMAC/DIABLO is the natural antagonist for XIAP and therefore promotes apoptosis. However, if XIAP is overexpressed, SMAC/DIABLO release from mitochondria sometimes is not sufficient to inhibit XIAP (Hinz et al. 2000; Fulda et al. 2002a).

Bcl-2 which maintains the integrity of the mitochondrial membrane has been shown to cause TRAIL resistance if highly expressed (Hinz et al. 2000; Fulda et al. 2002; Barnhart et al. 2003). Another Bcl-2 family member, myeloid cell leukemia-1 protein (Mcl-1), can inhibit BH-3 only proteins such as Bim, Bid, PUMA/NOXA and Bak (Adams and Cooper 2007) and can thus cause TRAIL resistance in certain type II cells (Taniai et al. 2004).

These different mechanisms of TRAIL resistance show the importance of understanding the interactions of different resistance mechanisms of TRAIL resistance in order to devise treatment that will effectively overcome them.

\subsection{The role of IAPs in TRAIL-induced apoptosis}

IAPs have been shown to be altered in many types of cancers and were found to correlate with chemoresistance, disease progression and poor prognosis (Hunter et al. 2007; LaCasse et al. 2008). This has lead to the rapid development of inhibitors of IAPs, referred to as SMAC mimetics, by different pharmaceutical companies which are currently in Phase I clinical trials for patients with locally advanced or metastatic malignancies e.g. (study number: NCT00977067). 
IAPs are defined by the presence of the baculovirus IAP repeat (BIR) domain, which is a zinc binding fold of $\sim 7$ - amino acid residues and is required for protein-protein interaction of IAPs (Hinds et al. 1999). IAPs, of which there are eight in humans, carry between one and three copies of this domain [figure 6]. The best studied IAPs cIAP-1, cIAP-2 and XIAP, each contain three BIR domains in their aminoterminal portion. Furthermore, for their Ubiquitin (Ub)-ligase (E3) activity they harbour a carboxyterminal RING (really interesting new gene) finger domain, through which they can interact with ubiquitin-conjugating enzymes (E2). Via the Ub-associated (UBA) domain IAPs are able to interact with ubiquitylated proteins. Furthermore, cIAP-1 and cIAP-2 also contain a CARD domain, however, its function is currently unknown.

IAPs are best known for their ability to regulate caspases and therefore block apoptosis. XIAP is the most potent inhibitor of caspase-3 and -7 in vivo and exerts its function via residues in the linker region between BIR1 and BIR2 domains that bind o the active site pocket of caspase-3 and -7 (Sun et al. 1999; Huang et al. 2001). For inhibition of caspase-9, the BIR3 domain of XIAP binds to the homodimerisation surface of caspase-9 interfering with caspase9 dimerisation which is essential for caspase-9 to become activated (Srinivasula et al. 2001).

Downregulation of XIAP by RNA interference has been shown to sensitise cells to apoptosis including ovarian carcinoma cell lines ( $\mathrm{Li} \mathrm{J}$ et al. 2000). It has also been shown to significantly enhance TRAIL-induced apoptosis in pancreatic carcinoma cells (Fulda and Debatin 2006; Vogler et al. 2007). In recent experiments it could be shown that SMAC mimetics sensitise cells to both, TRAIL- and TNF-induced apoptosis (Li L et al. 2004). Fulda et al. reported, that co-treatment of SMAC peptides and TRAIL lead to an eradication of preestablished glioma in a xenograft model in mice (Fulda et al. 2002b). Inactivation of IAPs with SMAC mimetic, even in combination with TRAIL, does not seem to negatively affect normal cells.

Even though SMAC mimetics were initially designed to antagonise XIAP, they have now also been shown to bind to cIAP-1 and cIAP-2 and to rapidly induce their auto-ubiquitination and proteasomal degradation. Levels of cIAP-1 and cIAP-2 are often high in cancer however, their role in mediating TRAIL resistance remains largely undefined.

Besides the involvement of cIAPs in apoptosis they also influence a multitude of other cellular processes, e.g. the ubiquitin-dependent activation of NF-kB (reviewed in (GyrdHansen and Meier 2010)). 


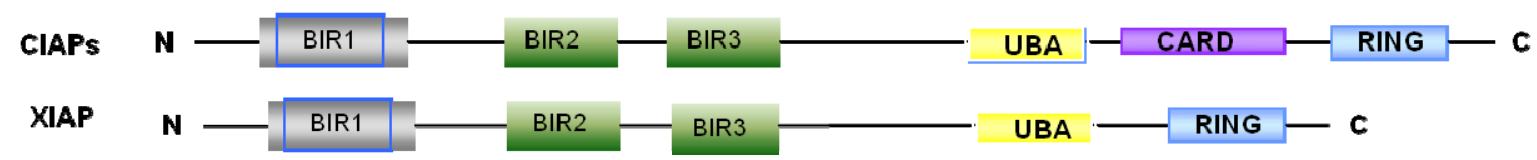

Figure 6: Family of IAPs. Baculovirus IAP repeat (BIR) domains enable interactions with proteins. BIR domains of IAPs can be grouped into type I (grey) and type II (green) BIR domains on the basis of the presence or abscense of a deep peptide binding groove. Type II BIR domains interact with caspases and IAP antagonists, whereas type I BIR domains interact with tumor necrosis factor receptor-associated factor 1 (TRAF1) and TRAF2. The Ubiquitin (Ub) binding domain UBA binds polyubiquitin (polyUb). The function of the CARD domain is currently unknown. The carboxy-termin RING domain is required for Ub ligase activity.

\subsection{Physiological role of TRAIL}

Until today, the physiological role and significance of TRAIL remains to be fully clarified. So far, no prominent role for TRAIL in mouse embryonic development could be shown, as (Cretney et al. 2002) TRAIL and also TRAIL-R knock out mice are viable, fertile and develop normally, except for an enlarged thymus (Diehl et al. 2004).

It has been assumed, that TRAIL holds a role in regulating and shaping the immune system, as TRAIL is selectively expressed on immune effector cells. However, no effect was seen in T and B-cells, macrophages, dendritic or natural killer cells, when TRAIL or mTRAIL-R were knocked out in mice. The immune system developed normally, and there was no defect in thymic negative selection. However, this latter finding is still controversial (Cretney et al. 2002; Lamhamedi-Cherradi et al. 2003). When mice were challenged with murine Cytomegalovirus (MCMV) in TRAIL-R deficient animals displayed enhanced resistance against MCMV challenge (Cretney et al. 2002).

Furthermore, it has been reported that TRAIL-deficient animals display increased severity in certain autoimmune disorders (Lamhamedi-Cherradi et al. 2003), like diabetes, arthritis (Song $\mathrm{K}$ et al. 2000), and multiple sclerosis (Cretney et al. 2005).

Recently, and most importantly for cancer research an immunosurveillance role for TRAIL has been suggested.

Grosse-Wilde and collegues (2008) were first to show the metastasis-specific surveillance function of TRAIL. Using an autochthonous DMBA/TPA (7.12-dimethylbenz $\{\alpha\}$ anthracene/ 12-O-tetradecanoylphorbol-13-acetate) induced skin carcinoma model, a 
significant increase in metastasis to the lymph nodes in mTRAIL-R knockout mice was observed, whereas the primary tumour growth rate was not affected. This indicates a role for TRAIL-Rs specifically in the suppression of metastasis, most likely because metastasising tumour cells are sensitised to TRAIL upon detachment via inhibition of the Erk pathway. In other cell types this may, however, be quite different as Trauzold and colleagues (Trauzold et al. 2006) reported a profound increase in metastatic spread of pancreatic carcinoma cells upon TRAIL treatment in vivo.

\subsection{TRAIL in clinical trials}

With the discovery of TRAIL and its ability to selectively induce apoptosis in tumour cells (Wiley et al. 1995) without harming normal cells (Ashkenazi et al. 1999; Walczak et al. 1999) a promising new therapeutic option for cancer treatment has evolved. At the moment, there are two different types of TRAIL-Receptor agonists in phase I and II clinical trials: agonistic TRAIL-R specific antibodies and recombinant soluble TRAIL. TRAIL-R specific antibodies have specifically been developed against TRAIL-R1 (e.g. Mapatumumab) and TRAIL-R2 (e.g. Lexatumumab, LBY135, Apomab, AMG-655). They are destined to evade the possible but controversial "decoy" function of TRAIL-R3 and TRAIL-R4 (LeBlanc and Ashkenazi 2003). TRAIL-R specific antibodies have a long half-life of 14-21 days, which can on the one hand potentiate their activity, but on the other hand, may also increase toxicity. Mapatumumab is however very well tolerated by patients and currently applied as monotherapy in phase II studies for heavily pretreated patients with Non-Hodgkin-Lymphoma (NHL), colorectal cancer (CRC) and non-small cell lung cancer (NSCLC). For NHL, 3 out of 40 patients showed objective clinical responses (Younes 2006). In CRC and NSCLC stable disease was reported with response rates of $32 \%$ and $29 \%$, respectively. Only one drug-related serious adverse event was recorded with the Mapatumumab mono-therapy.

As a lot of cell lines and most of the primary tumours are resistant to TRAIL-induced apoptosis they are effectively sensitised to TRAIL by different chemotherapeutic drugs and small molecule inhibitors. Another phase II study is now combining Mapatumumab with Bortezomib in advanced multiple myeloma in order to investigate its efficiency and safety (study number: HGS 1012-C1055). 
Similar results could be obtained with Lexatumumab, which is just as well tolerated and currently applied in phase Ia clinical trials.

Recombinant TRAIL can potentially bind to all five TRAIL-receptors and only as a half-live of about 30 minutes. However, as for its small size it is thought to be able to perfuse the tumour much more efficiently than the larger TRAIL-R antibodies.

For stronger apoptosis-inducing abilities of TRAIL, a tagged version of TRAIL is often used in in vitro assays, e.g. isoleucin zipper (iz) TRAIL, which keeps it in its physiological trimeric form. However, as a significant toxicity in human hepatocytes has been observed by Jo et al. (2003) using a His-tagged form of TRAIL (Jo et al. 2000), only untagged recombinant soluble TRAIL has entered the clinic. The toxicity of tagged TRAIL is still controversial, as another study by Ganten et al. (2005) showed, that only the His-tagged form is toxic to human hepatocytes but the iz-tagged form of TRAIL is not. Furthermore, they observed that freshly isolated human hepatocytes on day one of culture are susceptible to killing by TRAIL, however on day four, when their morphology resembles that of normal liver tissue much more readily, they are TRAIL- resistant.

Apo2/TRAIL is currently in phase Ib/ II clinical trials. Patients suffering from low-grade NHL received recombinant TRAIL either alone, or in combination with Rituximab. Recombinant TRAIL so far has proven to be active and save and no dose limiting toxicities have been reported. Furthermore, two out of five patients showed complete response, one partial response and two stable diseases.

In summary, it has been demonstrated that the application of recombinant TRAIL and TRAIL-R antibodies is very well tolerated in humans. TRAIL as a single agent therapy mainly caused stable disease or partial responses. However, it has to be considered that patients entering phase I/II clinical trials are often relapsed and are resistant to all conventional treatments available.

\subsection{Combined therapies of TRAIL and small molecule inhibitors}

As seen in clinical trials, TRAIL as a single agent therapy is effective, but still needs to be improved. Since primary cancer cells are often initially TRAIL resistant, co-treatment with a sensitising agent would make a TRAIL-based therapy much more efficient. Sensitising agents 
alter the levels of different anti-and/or pro-apoptotic molecules along the apoptotic pathway and therefore render cells more susceptible to TRAIL-induced cell death. Therefore, combined treatments of TRAIL plus small molecule inhibitors like Bortezomib are a very promising therapy option.

Bortezomib (Velcade $\AA$, PS-341) is a proteasome inhibitor that binds the catalytic site of the $26 \mathrm{~S}$ proteasome. It was recently approved by the FDA for the therapy of relapsed multiple myeloma and mantle cell lymphoma in the U.S.. Treatment of cancer cells with Bortezomib has been shown to have a variety of different effects, for instance inhibition of NF- $\mathrm{B}$ activation, inhibition of the cell cycle, changes in cell adherence and increased apoptosis induction (Adams et al. 1999; Adams 2001; Elliott and Ross 2001).

Combining proteasome inhibitors with TRAIL has been shown to sensitise a variety of different tumour cell lines and primary tumours to TRAIL- induced apoptosis, without being toxic to normal cells (Ganten et al. 2005; Koschny et al. 2007a). Only if the proteasome inhibitor was applied at a concentration 40 times as high as required for the sensitising effect, toxicity to human hepatocytes could be observed. Sensitisation of leukemia cells by Bortezomib appeared to involve downregulation of the anti-apoptotic protein cFLIP, while levels of Bcl-2 members and IAPs did not change (Sayers et al. 2003). Furthermore, it was reported that proteasome inhibitors promote activation of proapoptotic caspases such as caspase-3 (Zhang X. et al. 1999).

Combining proteasome inhibition with TRAIL has also been shown to reduce levels of XIAP in primary human keratinocytes, thus sensitising them to TRAIL-induced apoptosis (Leverkus et al. 2003).

Bortezomib-mediated sensitisation to TRAIL could now be shown for primary ovarian cancer cells, even for cisplatin and paclitaxel-chemoresistant ovarian tumours (Pasquini et al. 2010). A co-treatment of Bortezomib and Mapatumumab is currently applied to patients with advanced multiple myeloma in a phase II clinical trial.

Smac mimetics are small molecule inhibitors and were initially designed to mimic the structure of SMAC/DIABLO and thereby inhibit XIAP. Very soon, they were also found to target other members of the IAP family, especially cIAP-1 and cIAP-2 (Vince et al. 2007) and were found to lead to their degradation.

In the majority of cell lines tested SMAC mimetics alone only caused very little toxicity, however, if combined with TRAIL its apoptosis-inducing potency can be dramatically increased (Li L. et al. 2004). 
When it was shown, that activating mutations of certain kinases drive tumourigenesis, kinase inhibitors were intensively studied and rapidly introduced in different clinical trials. To date, 11 kinase inhibitors have received FDA approval as cancer treatments and approximately 30 distinct kinase inhibitors are currently applied in Phase I clinical trials.

As normal cells often tolerate inhibition of their kinase activity a therapeutic window for the selective killing of tumour cells has emerged.

Kinase inhibitors mostly target the ATP binding site, which is very well conserved within the kinase family pointing towards the difficulty of designing specific kinase inhibitors. However, selective kinase inhibitors were generated that can either target the active or inactive ATP binding state of their target kinase (Zhang J. et al. 2009).

Kinase inhibitors have been particularly successful in the treatment of chronic myeloid leukaemia (CML) where they could increase survival by several years (Druker et al. 2001; Heinrich et al. 2003). Mutation of the oncogenic breakpoint cluster region (BCR)-ABL1 is a causative transforming event in CML. Imatinib has been approved as first-line treatment for CML by the FDA and has replaced the previous standard treatment of $\alpha$-interferon and cytarabine (Deininger and Druker 2003). The side effect profile is much more favourable than conventional chemotherapy and exhibited up to $80 \%$ response rates in chronic phase CML patients (O'Brien et al. 2003).

Many kinase inhibitors have been shown to sensitise to TRAIL-induced apoptosis. For example the multi kinase inhibitor Sorafenib has entered clinical trials, not only as a single agent but also in combination with TRAIL (Ricci et al. 2007).

However, resistance against kinase inhibitors frequently occurs in the clinic and can either result from upregulation of alternative signalling pathways or mutations in the kinase that is under extreme selective pressure to acquire resistance through mutations in genes that abrogates drug binding. Therefore, it is important to develop inhibitors that target different kinase sites and discover treatments that act synergistically in combination with the kinase inhibitor.

\subsection{Ovarian cancer}

Ovarian cancer is the fifth most common cause of cancer death in women and the leading cause of death from gynaecological malignancies in the western world. About $75 \%$ of women are diagnosed in a late stage of the disease when the tumour has already spread beyond the ovaries (FIGO stage III and IV) so that they will require both surgical debulking and a 
systemic platinum-paclitaxel combination chemotherapy regimen as a standard first line therapy (Yap et al. 2009). Even though the survival rate has improved within the last years due to improved surgical techniques and empirically optimised chemotherapeutic regimes, the median progression free survival of these patients is still only 18 months (Greenlee et al. 2001).

While the initial responsiveness to platinum-based chemotherapy is still high, the majority of patients relapse with platinum resistant disease. Approximately $60 \%$ of patients still die within 5 years of diagnosis (Dinh et al. 2008). Epithelial ovarian cancer constitutes about 90\% of all ovarian tumours and can be subdivided into five major histopathological groups (high grade serous, low grade serous, endometrioid, clear cell and mucinous) [figure7]. They are thought to arise from ovarian surface epithelium (OSE) or from surface epithelial inclusion cysts (Feeley and Wells 2001). Many ovarian cancer cell lines and most of primary ovarian cancer cells are resistant to TRAIL-induced apoptosis, however, they can be sensitised to TRAIL-induced apoptosis with the addition of different small molecule inhibitors like chemotherapeutic drugs (Tomek et al. 2004) or Bortezomib (Koschny et al. 2007a).

\subsubsection{Frequent mutations in different ovarian cancer subtypes}

Even though the classification used for ovarian epithelial tumours is based entirely on tumour cell morphology, the distinct types are very likely to be associated with specific mutation pathways (Cho 2009). Serous carcinomas can be further divided into high-grade and lowgrade ovarian serous carcinomas. However, they have a different pathogenesis and should rather be seen as two distinct cancer types. A low-grade serous carcinoma only very rarely progresses into a high-grade carcinoma.

High-grade ovarian cancer most often presents with mutations or deletion in the TP53 gene (96.7\%) (Ahmed et al.2010), whereas they almost never occur in low-grade serous carcinomas. There is still a surprising degree of inconsistency between the relationship of TP53 mutations and clinical outcome. Limitations of many previous studys regarding the p53 status relies on p53 immunostaining instead of the sequencing of tumour DNA. Furthermore, inconsistencies between the studies might relate to whether the whole gene or just the most commonly mutated exons 5-8 were sequenced. A very recent study (Ahmed et al. 2010), sequenced the exons 2-11 and intron-exon boundaries in high grade ovarian carcinoma DNA from 145 patients with FIGO stage III and IV aiming to identify the true prevalence of TP53 
mutations in high grade serous ovarian carcinoma (HGSC). They could not determine an association between TP53 mutation and progression-free overall survival and conclude that TP53 is an important driver mutation in the pathogenesis of HGSC, however because TP53 mutations are almost invariably present in HGSC, it is not of substantial prognostic or predictive significance.

Nevertheless, apoptosis induction via TRAIL may be very beneficial as it is p53 independent and thus, remains largely unaffected by p53 mutations. TRAIL has even been shown to overcome cancer cell resistance to chemotherapy and to synergise with chemotherapy even in p53-deficient cells (Wissink et al. 2006).

Low-grade serous carcinomas are likely to harbour Kirsten rat sarcoma viral oncogene homolog (KRAS) or B-Raf proto-oncogenen serine-threonine/proteinkinase (BRAF) mutations which then lead to constitutive activation of the Mitogen-activated protein (MAP) kinase-pathway. Endometrioid ovarian carcinomas frequently hold mutations in the CTNNB1 gene that encodes for the beta-catenin protein and leads to activation of the Wnt-signalling pathway. When the Wnt pathway is activated beta-catenin is stabilised, translocates to the nucleus and activates downstream target genes (Cho and Shih Ie 2009). Endometrioid ovarian cancer also presents typically with TP53 mutations. Furthermore, typical mutations for endometrioid ovarian cancer are activating mutations in the Phosphoinositide 3-kinase (PI3K)/Akt pathway, either through activating mutations in the PI3K-alpha subunit itself, or silencing mutations of the counter regulator phosphatise and tensin homolog (PTEN). Clear cell ovarian carcinomas also frequently harbour PI3K-alpha activating mutations (Kuo et al. 2009). All of these pathways have been implicated in TRAIL-resistance.

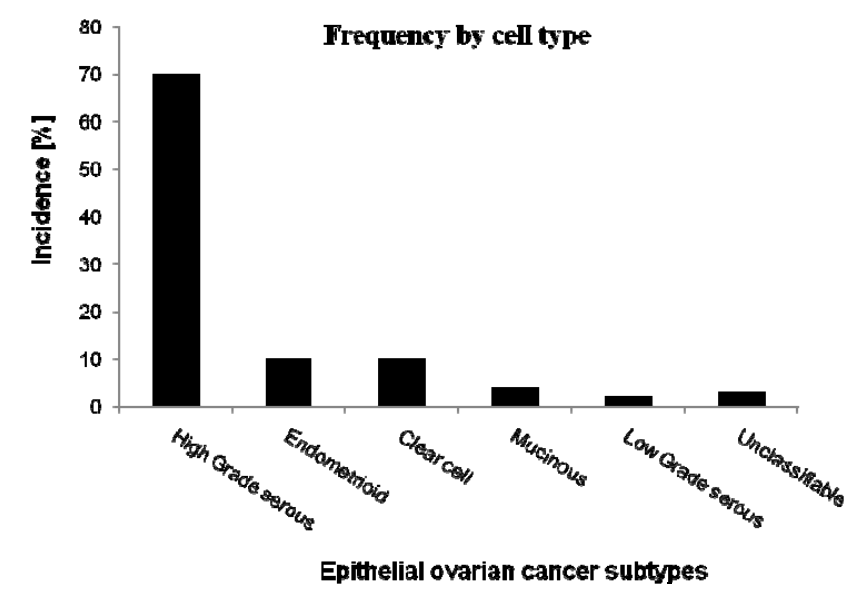

Figure 7: Frequency of different subtypes of ovarian carcinoma are shown.

Based on cases seen at the British Columbia Cancer Agency over the period 1986-2000. 


\subsection{EPCAM/CD326}

Epithelial-specific cell adhesion molecule (EpCAM/CD326) is a type I transmembrane glycoprotein of 39-42 kilo Dalton $(\mathrm{kDa})$, which is expressed by the majority of epithelial tissues and is overexpressed in some cancers, for instance colon, stomach prostate and lung cancers. (Baeuerle and Gires 2007; Carpenter and Red Brewer 2009). Approximately 70\% of ovarian cancer overexpress EpCAM. In contrast to cancer cells, that show a ubiquitous expression pattern, normal epithelial cells show a more polarised expression of EpCAM in tight junctions (Maetzel et al. 2009). Within the peritoneal cavity, EpCAM expression is tumour-specific because normal cells in the peritoneal compartment are of mesothelial origin and do not express EpCAM on their surface (Burges et al. 2007). Recently, it has been demonstrated, that EpCAM can be used as a cancer stem cell (CSC) marker. Using a model in which human breast cancer cells were grown in immunocompromised mice, Al-Hajj et al. (2003) demonstrated that the EpCAM+, CD44+, CD24- fraction of CSCs had a over tenfold higher frequency of tumour initiating cells, than the EpCAM-, CD44+, CD24-fraction (A1Hajj et al. 2003).

The EpCAM molecule is comprised of an extracellular domain with epidermal growth factor (EGF)-and thyroglobulin repeat-like domains, a single transmembrane domain, and a short 26-amino acid intracellular domain. EpCAM is a Calcium-independent adhesion molecule, which can antagonise E-Cadherin mediated cell-cell adhesion (Litvinov et al. 1994; Litvinov et al. 1997). Induction of EpCAM signalling induces the expression of certain target genes like c-myc, cyclin-A and cyclin-E, leading to increased cell proliferation (Munz et al. 2004). Furthermore, cells expressing EpCAM have also been shown to be less dependent on growth factors, as well as being able to survive anchorage-independently (Munz et al. 2004). Knock down of EpCAM in cells led to decreased proliferation and migration (Osta et al. 2004). Especially, the ability of cancer cells to survive anchorage independently is of course a crucial factor for their survival in ascitic fluid.

However, relatively little is known about the EpCAM signalling-pathway. Oligomerisation is a possible trigger for EpCAM activation, as treatment of cells with a recombinant form of the extracellular domain of EpCAM (EpEX) resulted in EpCAM cleavage (Baeuerle and Gires 2007). It could now be demonstrated that cleavage of EpCAM by ADAM metallopeptidase domain 17 (ADAM 17), also called TACE (tumour necrosis factor- $\alpha$-converting enzyme) 
releases EpEx and an intracellular fragment (EpICD). The intracellular fragment can now form a complex with beta-catenin, translocates to the nucleus and induces different target genes, including c-myc (Maetzel et al. 2009).

Since EpCAM is highly overexpressed in ovarian cancer and has been shown to be beneficial for anchorage-independent growth, it was selected in this thesis as a marker for ovarian cancer cells in ascitic fluid. 


\section{Aims and Objectives}




\section{Aims and Objectives}

The discovery of the tumour necrosis factor (TNF)-related-apoptosis-inducing ligand (TRAIL) more than 10 years ago was followed by intense research worldwide, as TRAIL was observed to kill tumour cells selectively without having toxic side effects on normal cells (Walczak et al. 1999). This distinguishes TRAIL from other apoptosis-inducing ligands such as $\mathrm{CD} 95 \mathrm{~L}$ which causes severe liver toxicity in mice and makes it highly interesting to investigate its potential for cancer therapy. However, it has recently become more apparent that $50 \%$ of all cell lines and the majority of human primary tumour cells are resistant to TRAIL-mediated apoptosis (Koschny et al. 2007b) and would need to be sensitised first. Ovarian cancer is the leading cause of death from gynaecological cancer and the fifth leading cause of death from cancer in women. Even though patients initially respond well to platinum-based chemotherapy, the majority of patients relapse with platinum-resistant disease. The response rate of resistant tumorous to standard treatments is often very poor and novel, more effective treatment options are urgently needed.

The aim of this thesis is to define agents that sensitise especially chemoresistant ovarian cancer cells to TRAIL-induced apoptosis.

1. Most importantly, the results obtained in ovarian cancer cell lines should be translated into primary ovarian cancer cells isolated from ascitic fluid. As compared to immortalised cell lines that have undergone many manipulation during their development results obtained from primary tumour cultures are much more likely to display the actual situation in the patient and are hence much more relevant for the clinical application.

2. Moreover, a further aim of this thesis is the analysis of molecular changes upon sensitisation. This is invaluable in order to understand the molecular mechanisms of TRAIL resistance and most importantly will lead to a more refined understanding of the biochemistry behind a treatment.

3. The long-term aim of understanding exactly which molecules are influenced upon different treatments will lead to a more patient-tailored therapy regime which can then fit the specific requirements for successful treatment of the individual patient. Due to restricted access to valuable primary material, this was ought to be studied in conventional cancer cell lines. 


\section{Material and Methods}




\section{Material and Methods}

\subsection{Material}

\subsubsection{Biological Material}

\section{Bacteria}

The E. coli strain AVB 101 (purchased from Avidity) was used for expression of moTAPTRAIL. The bacteria contain the biotinylating enzyme BirA in the expression vector paCYC (Chloramphenicol (Cam) resistance) which is inducible by IPTG.

\section{Cell lines}

\begin{tabular}{ll}
\hline Name & \multicolumn{1}{c}{ Description } \\
\hline HEY & human serous cell ovarian carcinoma \\
PEO-1 & human serous cell ovarian carcinoma \\
PEO-4 & human serous cell ovarian carcinoma \\
PEA-1 & human serous cell ovarian carcinoma \\
PEA-2 & human serous cell ovarian carcinoma \\
SKOV-3 & human serous cell ovarian carcinoma \\
& \\
KK & human clear cell ovarian carcinoma \\
TOV-112D & human endometrioid ovarian carcinoma \\
IGROV-1 & human undifferenciated ovarian carcinoma \\
IOSE-80 & human undifferenciated ovarian carcinoma \\
DKO4 & human colon cancer cell line \\
DKO4dnFADD & human colon cancer cell line
\end{tabular}


Human ovarian carcinoma cell lines were a kind gift from H. Gabra, Imperial College London

DKO4 and DKO4 dn FADD were a kind gift from O. Kranenburg, UMC Utrecht; The Netherlands

\subsubsection{Culture Media}

Human ovarian carcinoma cell lines and primary ovarian cancer cells were kept in RPMI 1640 (Gibco/Invitrogen, Karlsruhe, Germany) containing Glutamax I, a more stable Glutamine source, and supplemented with 10\% FCS (Biochrom AG, Berlin, Germany) and 1\% Penicillin/Streptomycin.

DKO4 and DKO4 dnFADD cells were kept in DMEM (Gibco/Invitrogen, Karlsruhe, Germany) supplemented with 10\% FCS (Biochrom AG, Berlin, Germany) and 2mM Glutamine.

Bacterial culture was maintained in LB-medium containing Ampicillin $(30 \mu \mathrm{g} / \mathrm{ml})$ and Chloramphenicol $(100 \mu \mathrm{g} / \mathrm{ml})$. Thereby, selection for two different plasmids was achieved.

\subsubsection{Antibodies}

\section{For Flow Cytometry}

For Flow Cytometry following antibodies were used:

Fluorescently labelled primary antibodies:

\begin{tabular}{llll} 
Antibody & Antigen & Isotype & Supplier, Reference \\
\hline Anti-Human Epithelial Ag -FITC & Ber-Ep4 & mIgG1 & DAKO \\
Anti HumanCD45 -APC & 5B1 & mIgG2a & Miltenyi Biotech \\
Anti Human CD14-FITC & MEM-18 & mIgG1 & AbD serotec
\end{tabular}


Fluorescently labelled Isotype controls:

\begin{tabular}{lll} 
Antibody & Isotype & Supplier, Reference \\
\hline FITC Mouse & IgG1 & eBioscience Ltd \\
APC mouse & IgG1 & Immuno Tools
\end{tabular}

\begin{tabular}{lll} 
Antibody & Isotype & Supplier, Reference \\
\hline CD16/32 Fc-block & IgG1 & eBioscience Ltd
\end{tabular}

Uncoupled monoclonal antibodies (primary antibodies)

\begin{tabular}{llcl} 
Antibody & Antigen & Isotype & Supplier, Reference \\
\hline HS101 & TRAIL-R1 & mIgG1 & Alexis \\
HS201 & TRAIL-R2 & mIgG1 & Alexis
\end{tabular}

Biotin-coupled antibody (secondary antibody)

\begin{tabular}{llll} 
Antibody & Target & Serum & Supplier, Reference \\
\hline anti-IgG1-Bio mIgG1 & Goat & BD Pharmingen
\end{tabular}

Fluorescently-labelled tertiary detecting molecules

\begin{tabular}{llll} 
Basic Protein & Fluorescent Dye & Target & Supplier, Reference \\
\hline Streptavidin & Phycoerythrin (PE) & Biotin & BD Pharmingen
\end{tabular}




\section{For Western Blot}

For Western Blot analysis, following primary antibodies were used:

\begin{tabular}{|c|c|c|c|}
\hline Antibody & Clone & Isotype & Supplier, Reference \\
\hline$\beta$-Actin & $\begin{array}{l}\mathrm{AC}-15 \\
(\mathrm{mAb})\end{array}$ & mIgG1 & $\begin{array}{l}\text { Sigma } \\
\text { Gillingham,UK }\end{array}$ \\
\hline Bid & $\begin{array}{l}550365 \\
(\mathrm{pAb})\end{array}$ & Rabbit IgG & $\begin{array}{l}\text { BD Pharmingen } \\
\text { Hamburg, Germany }\end{array}$ \\
\hline His-tag & $\begin{array}{l}\text { BMG-His } 1 \\
(\mathrm{mAb})\end{array}$ & mIgG1 & Roche Applied Sience \\
\hline Caspase-3 & $\begin{array}{l}\text { CPP32 } \\
(\mathrm{pAb})\end{array}$ & Goat IgG & $\begin{array}{l}\text { R\&D Systems } \\
\text { Abingdon,UK }\end{array}$ \\
\hline Caspase-8 & $\begin{array}{l}\mathrm{C} 15 \\
(\mathrm{mAb})\end{array}$ & $\operatorname{IgG} 2 b$ & $\begin{array}{l}\text { Axxora } \\
\text { San Diego, CA }\end{array}$ \\
\hline Caspase-9 & $\begin{array}{l}5 \mathrm{~B} 4 \\
(\mathrm{mAb})\end{array}$ & mIgG1 & $\begin{array}{l}\text { MBL } \\
\text { Watertown, MA }\end{array}$ \\
\hline cFLIP & $\begin{array}{l}\text { NF-6 } \\
(\mathrm{mAb})\end{array}$ & mIgG1 & $\begin{array}{l}\text { Axxora } \\
\text { San Diego, CA }\end{array}$ \\
\hline cIAP-1 & $\begin{array}{l}\text { AF8181 } \\
(\mathrm{mAb})\end{array}$ & Goat IgG & $\begin{array}{l}\text { R\&D Systems } \\
\text { Abingdon,UK }\end{array}$ \\
\hline cIAP-2 & $\begin{array}{l}208 \\
(\mathrm{mAb})\end{array}$ & Rat IgG1 & Kindly donated by J.Silke \\
\hline cIAP pan & $\begin{array}{l}\text { MAB3400 } \\
(\mathrm{mAb})\end{array}$ & $\operatorname{IgG} 2 \mathrm{a}$ & $\begin{array}{l}\text { R\&D Systems } \\
\text { Abingdon,UK }\end{array}$ \\
\hline FADD & $\begin{array}{l}556402 \\
(\mathrm{mAb})\end{array}$ & IgG1 & $\begin{array}{l}\text { BD Pharmingen } \\
\text { Hamburg, Germany }\end{array}$ \\
\hline FLAG & $\begin{array}{l}\mathrm{M} 2 \\
(\mathrm{mAb})\end{array}$ & $\operatorname{IgG1}$ & $\begin{array}{l}\text { Sigma } \\
\text { Gillingham,UK }\end{array}$ \\
\hline PARP & $\begin{array}{l}\mathrm{C}-2-10 \\
(\mathrm{mAb})\end{array}$ & IgG1 & $\begin{array}{l}\text { Biomol } \\
\text { Hamburg, Germany }\end{array}$ \\
\hline TRAIL-R1 & $\begin{array}{l}\text { PSC-1139 } \\
(\mathrm{pAb})\end{array}$ & Rabbit & $\begin{array}{l}\text { Axxora } \\
\text { San Diego, CA }\end{array}$ \\
\hline TRAIL-R2 & $\begin{array}{l}\text { PSC-2019 } \\
(\mathrm{pAb})\end{array}$ & Rabbit & $\begin{array}{l}\text { Axxora } \\
\text { San Diego, CA }\end{array}$ \\
\hline XIAP & $\begin{array}{l}610762 \\
(\mathrm{mAb})\end{array}$ & IgG1 & $\begin{array}{l}\text { BD Pharmingen } \\
\text { Hamburg, Germany }\end{array}$ \\
\hline
\end{tabular}


Horseradish peroxidase coupled antibodies (secondary antibodies)

$\begin{array}{llll}\text { Antibody } & \text { Target } & \text { Serum } & \text { Supplier, Reference } \\ \text { anti-mIgG1-Hrp } & \text { mIgG1 } & \text { Goat } & \text { Southern Biotech } \\ \text { anti-mIgG2b-Hrp } & \text { mIgG2b } & \text { Goat } & \text { Southern Biotech } \\ \text { anti-goat IgG-Hrp } & \text { goat IgG } & \text { Rabbit } & \text { Santa Cruz } \\ \text { anti-rabbit IgG-Hrp } & \text { rabbit IgG } & \text { Goat } & \text { Southern Biotech }\end{array}$

Horseradish peroxidase coupled detecting molecules

$\begin{array}{llll}\text { Basic Protein Enzyme } & \text { Enzyme } & \text { Target } & \text { Supplier, Reference } \\ \text { Streptavidin } & \text { HRP } & \text { Biotin } & \text { BD Pharmingen }\end{array}$

\subsubsection{Chemotherapeutics and Inhibitors}

$\begin{array}{ll}\text { Agent } & \text { Supplier, Reference } \\ \text { Cisplatin } & \text { Merck } \\ \text { PS-341 } & \text { Sigma } \\ \text { Smac83/59 } & \text { D.Delia; Dep. of exp. Oncology Milan, Italy } \\ \text { PIK75 } & \text { Calbiochem }\end{array}$

\subsubsection{Ligands}

For sensitisation experiments an N-terminally isoleucine-zipper tagged form of recombinant human TRAIL (iz-TRAIL) was used which has been shown to exert very high activity (Ganten et al. 2006). This iz-TRAIL had been produced in E. coli and purified in a two step purification using a hydroxyapatite column and subsequently a Ni- NTA column as established in our lab.

For immune-precipitation recombinant human TRAIL (extracellular domain) was produced containing a modified Tandem Affinity Purification (moTAP) tag (Haas et al. 2009). 
3.1.6. Plasmids

Plasmid

CD95-Fc

TRAIL-R2-Fc

TNF-R2-Fc

pcDNA 3.1. Caspase-8

\section{Supplier}

Kindly provided by D.Willen

Kindly provided by D.Willen

Kindly provided by E.Rieser

Kindly provided by C.Falschlehner

\subsubsection{Chemicals}

\section{Chemical}

Acetic acid (HOAc)

Biotin

Calciumchlorid $\left(\mathrm{CaCl}_{2}\right)$

CD45 Dynabeads ${ }^{\circledR}$

Coomassie Brilliant Blue (G-250)

Bovine serumalbumin (BSA)

Dimethylsulfoxid (DMSO)

Dinatriumhydrogenphosphat $\left(\mathrm{Na}_{2} \mathrm{HPO}_{4} \cdot 2 \mathrm{H}_{2} \mathrm{O}\right)$

EpCAM Dynabeads ${ }^{\circledR}$

Ethanol absolut (EtOH)

Ethylendiamintetraacetat (EDTA)

Fetales Kälberserum (FCS)

Ficoll-Paque Plus

Formaldehyd

Glycin

Glycerol

HEPES

Hydrochloric acid ( $\mathrm{HCl})$

Isopropanole

IPTG

Kaliumacetat (KOAc)

Kaliumchlorid ( $\mathrm{KCl})$

Kaliumdihydrogenphosphat $\left(\mathrm{KH}_{2} \mathrm{PO}_{4}\right)$

Kaliumhydrogencarbonat $\left(\mathrm{KHCO}_{3}\right)$

L-Glutamine

Lipofectamine2000

\section{Supplier}

J. T. Baker Chemicals

Pierce

Merck

Invitrogen

Merck

Serva, Heidelberg

Serva

Merck

Invitrogen

Riedel-de Häen

Merck, Darmstadt

Gibco BRL

Gibco BRL

Merck

Sigma

USB

Gibco BRL

J. T. Baker Chemicals

Roth

Sigma

Riedel-de Häen

Merck

Merck

Merck

Invitrogen

Invitrogen 
Magnesiumchlorid $\left(\mathrm{MgCl}_{2} \cdot 6 \mathrm{H}_{2} \mathrm{O}\right)$

Merck

$\beta$-Mercaptoethanol ( $\beta$-ME)

Sigma

Methanole $(\mathrm{MeOH})$

Riedel-de Häen

MES (Morphilino Ethan Sulfonsäure)

USB

Milk powder

Fluka Chemika

MTT (methoxy-tetrazolium salt)

Sigma

Natriumacetat ( $\mathrm{NaOAc}$ )

Merck

Natriumazid $\left(\mathrm{NaN}_{3}\right)$

Merck

Natriumchlorid $(\mathrm{NaCl})$

Sigma

Natriumcitrat

Sigma

Natriumdodecylsulfat (SDS)

Sigma

Natriumhydrogencarbonat $\left(\mathrm{NaHCO}_{3}\right)$

Merck

Natriumhydroxid $(\mathrm{NaOH})$

Merck

Nickel-NTA-Sepharose FF

Qiagen

Pluronic F-77

Sigma

Polyethylenglykol 1500

Roche

Ponceau S

Serva

Propidium-iodide

Sigma

Protease Inhibitors

Sigma

RBC Lysis buffer

eBioscience Ltd

SuperSignal West Dura Extended

Pierce, Rockford; USA

SuperSignal West Femto Extended

Pierce, Rockford; USA

TCEP $^{\circledR}$ Bond Breaker

Pierce

Tris-Hydrochloride (Tris-HCl)

Sigma

Triton X-100

USB

Trizma Base

Sigma

10 x Trypsin

Gibco BRL

Tween 20

USB

Western Lightning-ECL

PerkinElmer,

Massachusetts; USA

\subsubsection{Buffers and Solutions}

Bacteria lysis buffer

$50 \mathrm{mM}$ Tris $-\mathrm{HCl} \mathrm{pH} 7,4$

$200 \mathrm{mM} \mathrm{NaCl}$

$100 \mathrm{mM} \mathrm{KCl}$

$10 \%$ Glycerol

$0,5 \%$ Triton $\mathrm{x} 100$ 
Blocking Milk (Western Blot)

Cell lysis buffer

Column wash buffer

Column elution buffer

FACS-Buffer

IP-Lysis-Buffer:
Prior to use add $2 \mathrm{mM} \beta$ - Mercaptoethanol $1 \times$ PBS

$5 \%$ Milk powder

$0.05 \%$ Tween-20

$30 \mathrm{mM}$ Tris- $\mathrm{HCl} \mathrm{pH} 7.5$

$150 \mathrm{mM} \mathrm{NaCl}$

$10 \%$ Glycerol

$1 \%$ Triton $\mathrm{X}-100$

50 mM Tris- $\mathrm{HCl} \mathrm{pH} 8$

$300 \mathrm{mM} \mathrm{NaCl}$

$15 \mathrm{mM}$ Imidazole

Prior to use add $2 \mathrm{mM} \beta$ - Mercaptoethanol

$50 \mathrm{mM}$ Tris- $\mathrm{HCl} \mathrm{pH} 8$

$300 \mathrm{mM} \mathrm{NaCl}$

$170 \mathrm{mM}$ Imidazole

Prior to use add $2 \mathrm{mM} \beta$ - Mercaptoethanol

1x PBS

$5 \%$ FCS

$30 \mathrm{mM}$ Tris- $\mathrm{HCl}, \mathrm{pH} 7,4$ at $0{ }^{\circ} \mathrm{C}$

$120 \mathrm{mM} \mathrm{NaCl}$

2 mM EDTA

$2 \mathrm{mM} \mathrm{KCl}$

$10 \%$ Glycerol (v/v)

$1 \%$ Triton $\mathrm{X}-100$ 
Maintenance Buffer

MES Running Buffer $(20 \times)$

MOPS Running Buffer (20×)

MTT developing solution

Nicoletti-buffer:

$1 \times \mathrm{PBS}$

PI solution
$50 \mathrm{mM}$ Trizma Base

$100 \mathrm{mM} \mathrm{NaCl}$

$0,02 \%$ Tween 20

0,5 M L-Arginin

2 mM DTT

$50 \mathrm{mM}$ MES

$50 \mathrm{mM}$ Trizma Base

1 mM EDTA

$0.1 \%(\mathrm{w} / \mathrm{v}) \mathrm{SDS}$

\section{0mM MOPS}

50mM Trizma Base

$1 \mathrm{mM}$ EDTA

$0.1 \%(\mathrm{w} / \mathrm{v}) \mathrm{SDS}$

Isopropanole

5\% Acetic acid

$0,1 \%$ Natriumcitrat (w/v)

$0,1 \%$ Triton X-100 (w/v)

$50 \mu \mathrm{g} / \mathrm{ml}$ Propidium-Iodide

$137 \mathrm{mM} \mathrm{NaCl}$

$2.7 \mathrm{mM} \mathrm{KCl}$

$8.1 \mathrm{mM} \mathrm{Na}_{2} \mathrm{HPO}_{4}$

$1.5 \mathrm{mM} \mathrm{KH}_{2} \mathrm{PO}_{4}$

$1 \mu \mathrm{g} / \mathrm{ml}$ Propidium-Iodide

$1 \times$ PBS 
6 x SDS PAGE sample buffer

Stripping buffer (Western Blot)

Transfer Buffer (Western Blot)

PBS/Tween (Western Blot)
$0.35 \mathrm{M}$ Tris- $\mathrm{HCl}(\mathrm{pH} 6.8)$

$10.28 \%$ SDS

$36 \%$ Glycerol

0.012\% Bromophenol Blue

50 mM Glycine $\mathrm{HCl}$ pH 2.3

192 mM Glycine

$25 \mathrm{mM}$ Tris

$0.01 \%$ SDS

$20 \%$ Methanol

$1 \times$ PBS

$0.05 \%$ Tween-20

\subsubsection{Consumables}

Cell Culture Petri dishes

Cell Culture Test Plates (6-, 12-, 24-well)

DynaMag $^{\text {TM}}-2$

Round and flat bottom 96-well test plates

Tissue Culture flasks (25 and $75 \mathrm{~cm} 2$ )

Falcons $(15 \mathrm{ml}$ and $50 \mathrm{ml})$

Plastic pipettes $(5 \mathrm{ml}, 10 \mathrm{ml}$ and $25 \mathrm{ml})$

Pipette tips $(0.1-10,1-200,101-1000 \mu \mathrm{l})$

Safe-Lock Reaction Tubes (1,5ml, 2 ml)

Sealing foil

NuPAGE® 4-12\% Bis-Tris Gels

Hybond ECL Nitrocellulose Membrane

X-Ray film HyperfilmTM ECL

Whatman paper
TPP, Trasadingen; Switzerland

TPP, Trasadingen; Switzerland Invitrogen; UK

TPP, Trasadingen; Switzerland TPP, Trasadingen; Switzerland TPP, Trasadingen; Switzerland Becton Dickinson, Heidelberg; Germany StarLab, Ahrensburg, Germany Eppendorf, Hamburg; Germany Roche, Mannheim; Germany Invitrogen, Karlsruhe; Germany Amersham Bioscience; UK Amersham, Freiburg; Germany Schleicher\&Schuell; UK 
Polypropylene round bottom tube $(10 \mathrm{ml})$ Becton Dickinson, Heidelberg;Germany

Cuvette

Greiner Bio-One, Flacht; Germany

Dialysis Tube

Roth, Karlsruhe; Germany

PS- Test Tubes for FACS

Greiner Bio-One, Flacht; Germany

Cryogenic vials

Nunc, Wiesbaden; Germany

Single-Use Syringe ( $5 \mathrm{ml}, 30 \mathrm{ml}, 50 \mathrm{ml})$

Terumo, Eschborn; Germany

Sterile filter $(0.22 \mu \mathrm{m}$ and $0,45 \mu \mathrm{m}$ pore size $)$ Millipore, Billerica; USA

Single-Use Scalpel

Single-Use Needles

Glassware

X-Ray film Hyperfilm TM ECL
Feather, Osaka; Japan

Becton Dickinson, Heidelberg;Germany

Schott, Mainz; Germany

Amersham Bioscience ;UK

\subsubsection{Instruments}

Äkta Prime

Flow Cytometer FACSCalibur

Multiskan Ascent

Hyper Processor X-Ray film Developer

Blotting equipment $\mathrm{X}$ cell $\mathrm{II}^{\mathrm{TM}}$

NanoDrop Spectrophotometer ND-1000

Photometer Ultrospec 3100 pro

Biohazard safety cabinet class II

Incubator Stericult 2000

Microwave

Table Centrifuge Biofuge

Varifuge 3O-R

Multifuge 3S-R

Biofuge Stratos

Power Supply for agarose gels

Light Microscope

Vortex

Cryo $1^{\circ} \mathrm{C}$ Freezing container $\mathrm{pH}$ Meter
Amersham Pharmacia Biotech, Austria

Becton Dickinson, Heidelberg; Germany

Thermo Labsystems, Vantaa; Finnland

Amersham Bioscience; UK

Novex, Bergisch Gladbach; Germany

NanoDrop Technologies, USA

Amersham, Freiburg; Germany

Scanlaf, Lynge, Denmark

Forma Scientific, Scotia; USA

AEG, Nuremberg; Germany

Heraeus, Hanau, Germany

Heraeus, Hanau, Germany

Heraeus, Hanau, Germany

Heraeus, Hanau, Germany

BioRad, Hercules; USA

Zeiss, Oberkochen; Germany

Heidolph, Schwabach; Germany

Nalgene Labware, Neerijse; Belgium

Mettler, Giessen; Germany 
Thermomixer compact

Freezer $-20^{\circ} \mathrm{C}$

Freezer $-80^{\circ} \mathrm{C}$

GelSystem Flexi 4040

Pipettes $(10 \mu \mathrm{l}, 100 \mu \mathrm{l}, 200 \mu \mathrm{l}, 1 \mathrm{ml})$

Pipetboy

Multichannel pipettes

Multistepper

Multitron Incubator Shaker

See-Saw Rocker

Sonifier

Water bath
Eppendorf, Hamburg; Germany

Liebherr, Biberach; Germany

New Brunswick Scientific Co; USA

Biostep, Jahnsdorf; Germany

Gilson, Bad Camber; Germany

Integra Bioscience, Fernwald;

Micronic Systems; USA

Eppendorf, Hamburg; Germany

Appropriate Technical Resources; USA

Stuart;XXX

Branson Ultrasonics Corporation, USA

B.Braun, Melsungen: Germany

\subsection{Methods}

\subsubsection{Cellular Biology Methods}

\section{Cell Culture and passaging of adherent cells}

All cell lines were cultured in $75 \mathrm{~cm}^{2}$ or $150 \mathrm{~cm}^{2}$ flasks (TPP, Helena Bioscience).

Cells were passaged every three to four days, at cell densities around $5 \times 10^{6}$ cells $\left(75 \mathrm{~cm}^{2}\right.$ flask) or $1 \times 10^{7}$ cells $\left(150 \mathrm{~cm}^{2}\right.$ flasks). They were initially washed $1-2$ times with $1 \times \mathrm{PBS}$ and then detached from the surface of the culture flasks by incubation with $5-10 \mathrm{ml} 1 \times$ Trypsin for $1-5$ min at $37^{\circ} \mathrm{C}$. Afterwards, fresh medium containing $10 \%$ FCS was added to stop the action of trypsin. Detached cells were transferred to a falcon tube, centrifuged and resuspended in fresh medium containing 10\% FCS. Depending on cell density and growing behaviour, cells were usually split at a ratio of 1:10 to 1:3 and transferred into new culture flasks. All handling of cells was performed under sterile conditions to avoid contamination with prokaryotes. 


\section{Counting of Cells}

To determine the number of cells/ml, adherent cells were detached with trypsin and resuspended in fresh medium containing $10 \%$ FCS as described before. $20 \mu 1$ of this cell suspension were diluted with $20 \mu 1$ trypane blue and applied to a "Neubauer counting chamber" [figure 7]. Trypane blue only penetrates the cell wall of dead cells. All trypane blue negative cells in the four outer big squares were counted and divided by four. This number is then multiplied by two, as the cell suspension was dilutes $1: 2$ by trypane blue. Therefore, the cell number per $\mathrm{ml}$ is calculated by following formula:

$(2 \times$ mean trypane negative cell number per big square $) \times 10^{4}=$ cells $/ \mathrm{ml}$

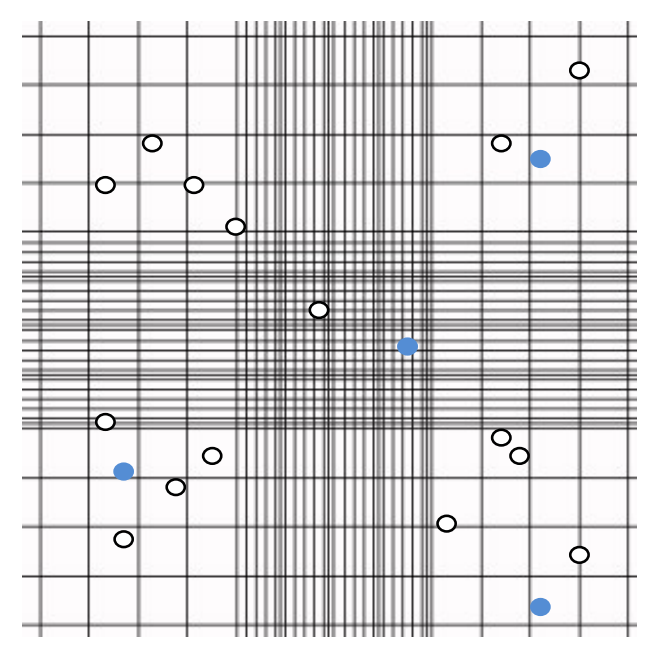

Figure 7: Neubauer counting chamber. Trypane negative cells are counted in the 4 outer squares. In this example 13 cells would be counted. The counted cell number is then divided by four (= mean cell number by square), which is 3.25 in this example. This number is then multiplied by two, as the cell suspension was diluted $1: 2$ by trypane blue, in this example 6,5 . Therfore the cell number present in the cell suspension is $6,5 \times 10^{4}$ cells $/ \mathrm{ml}$.

\section{Freezing and thawing of cells}

To freeze eukaryotic cell lines, adherent cells were detached from the flasks as described before. After centrifugation, cells were re-suspended in pre-cooled $\left(+4{ }^{\circ} \mathrm{C}\right) \mathrm{FCS}$ containing $10 \%$ DMSO and aliquoted into cryotubes $\left(5 \times 10^{6}-1 \times 10^{7}\right.$ cells $\left./ \mathrm{ml}\right)$. DMSO was used as a cryoprotectant because it prevents the formation of ice crystals which otherwise would lyse the cells during thawing. The cells were slowly cooled to $-80{ }^{\circ} \mathrm{C}$ and then transferred to the liquid nitrogen tank where they were kept for long-term storage at $-196{ }^{\circ} \mathrm{C}$. 
To take frozen cells into culture, cells were thawed at $37^{\circ} \mathrm{C}$ and rapidly transferred to a cell culture flask and $15 \mathrm{ml}$ prewarmed $\left(37^{\circ} \mathrm{C}\right)$ medium containing $10 \%$ FCS was added. After attachment of the cells the medium was replaced by prewarmed fresh medium containing 10\% FCS and cells were cultured at $37{ }^{\circ} \mathrm{C}$ in a humidified atmosphere with $5 \% \mathrm{CO}_{2}$.

\section{Seeding cells into Cell Culture Test Plates}

For seeding cells into cell culture test plates they had to be counted as described above and then the respective volume was taken containing the desired number of cells. All single toxicity and sensitisation experiments were performed in 96-well test plates which were seeded at $1 \times 10^{4}$ cells per well in $100 \mu \mathrm{l}$ each. The apoptosis assays were performed in 12 well test plates containing $1 \times 10^{5}$ cells per well in $1 \mathrm{ml}$ and the lysates for investigation of changes in intracellular proteins were made from cells in 6 well test plates with $2 \times 10^{5}$ cells per well seeded in $2 \mathrm{ml}$. After seeding all test plates were left to incubate over night to let cells adhere properly before using them in an experiment.

\section{Ficoll}

Ficoll-Paque PLUS was used to separate erythrocytes from leucocytes and cancer cells in the ascitic fluid.

Usually, one gradient was used for each $250 \mathrm{ml}$ of ascites. The cell pellet was taken up in 5$10 \mathrm{ml}$ ascitic fluid supernatant (SN) and was then carefully layered on the Ficoll-Paque Plus of which the same volume was used without mixing both components. The sample was centrifuged at $400 \times \mathrm{g}$ for 20 minutes at $18-20^{\circ} \mathrm{C}$. The upper $\mathrm{SN}$ layer was taken off with a clean Pasteur pipette, leaving the lymphocyte/cancer cell layer undisturbed at the interface. The lymphocyte/cancer cell layer was transferred to a clean centrifuge tube with prewarmed RPMI medium. 


\section{Cell viability assays}

\section{Methoxy Tetrazolium salt (MTT)}

24h after application of TRAIL $25 \mu \mathrm{l}$ of MTT (3-(4,5- Dimethylthiazol-2-yl)-2,5dilphenyltetrazolium bromide) reagent $(2,5 \mathrm{mg} / \mathrm{ml}$ in PBS) was added to each well (96 well plate) and incubated at $37^{\circ} \mathrm{C}$ for $2 \mathrm{~h}$. During the incubation the yellow MTT is reduced to purple formazan by mitochondrial reductase enzymes which are only active when the cell is viable. However, one should bear in mind when working with MTT that some treatments can slow down cell metabolism and therefore evoke the false impression of non viable cells. The medium was then removed using a vacuum pump and MTT developing solution was added to each well solving the purple formazan cristals inside those cells which were alive at the time MTT solution was added. After letting the plates shake for $15 \mathrm{~min}$ absorption was measured using a $450 \mathrm{~nm}$ filter at the Multiskan Ascent (Thermo Labsystems, Vantaa; Finnland).

\section{Cell titer glo®}

The cell titer glo® assay relies on the quantification of ATP present in order to determine the number of viable cells.

24h, after TRAIL treatment 96 well plates were spun down for 3 min 1400 RPM. SN was disposed and $100 \mu 1$ of a 1:4 with $1 \times$ PBS diluted mixture of cell titer glo ${ }^{\circ}$ reagent was applied to the cells. This results in lysis and generation of a luminescent signal proportional to the amount of ATP present. The amount of ATP is directly proportional to the number of cells present in culture.

After $2 \mathrm{~min}$. shaking followed by $10 \mathrm{~min}$. incubation in order to allow for stabilisation of the luminescence signal, luminescence was measured using Mithras LB940.

\section{Quantification of Apoptosis}

As a direct measurement of apoptotic cell death, DNA fragmentation was quantified as described (Nicoletti et al. 1991). Briefly, $0.5 \times 10^{5}$ cells were seeded in 24 -well plates. On the next day they were incubated with or without apoptotic stimulus in $1 \mathrm{ml}$ medium at $37^{\circ} \mathrm{C}$ for $24 \mathrm{~h}$ or $48 \mathrm{~h}$. Living and dead cells were harvested in the same time, washed twice with PBS and then resuspended in $300 \mu 1$ "nicoletti buffer" (see buffers). After $24 \mathrm{~h}$ incubation at $4{ }^{\circ} \mathrm{C}$ 
apoptosis was quantitatively determined as cells containing nuclei with subdiploid DNA content using flow cytometry.

A typical Histogram plotting PI fluorescence intensity against nuclei number is shown in figure 8 .

A

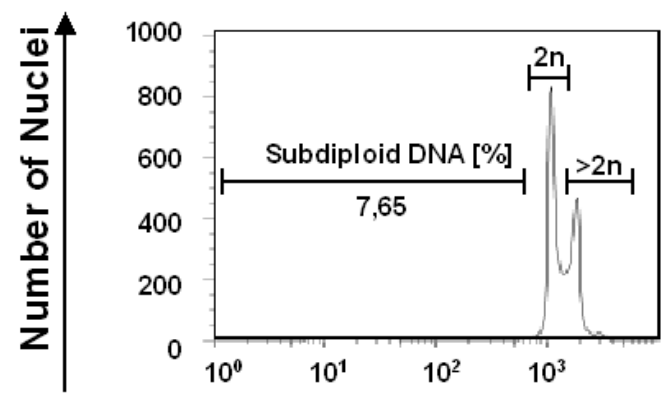

B

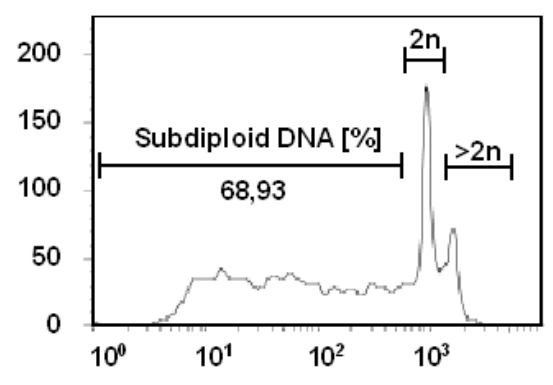

Fluorescence intensitiy (PI)

Figure 8. Characteristic histograms of living and apoptotic cells. A. Living cells show a typical G1 peak which is the highest, and the G2/M-phase peak separated by intermediate DNA content in the S-phase. Cells were only incubated with medium. B. Apoptotic cells fragment their DNA which can be seen in the histogram as an increase in subdiploid DNA content. These cells were treated with $500 \mathrm{ng} / \mathrm{ml}$ iz-TRAIL for $24 \mathrm{~h}$.

\section{Transfection of adherent cells}

TOV-112D cells were transfected with Lipofectamine2000 according to the manufacturer's protocol.

\subsubsection{Biochemical Methods}

\section{Preparation of cell lysates}

Ovarian carcinoma cell lines were detached from the plates by trypsin and taken up into medium and transferred to $1.5 \mathrm{ml}$ Eppendorf tubes. Cells were harvested by centrifugation at $4000 \mathrm{rpm}$ for $5 \mathrm{~min}$ at $4^{\circ} \mathrm{C}$, washed twice with $1 \times \mathrm{PBS}$ and lysates were prepared by 
resuspending the resulting cell pellets in $50 \mu$ lysis buffer $(30 \mathrm{mM}$ Tris- $\mathrm{HCl} \mathrm{pH} 7.5,150 \mathrm{mM}$ $\mathrm{NaCl}, 10 \%$ glycerol 1\% Triton $\mathrm{X}-100$ ) supplemented with Complete ${ }^{\mathrm{TM}}$ protease inhibitors (Roche Diagnostics, Mannheim, Germany) according to the manufacturer's instructions. After 30 min incubation on ice, the lysates were centrifuged at $15000 \mathrm{rpm}$ for $30 \mathrm{~min}$ at $4^{\circ} \mathrm{C}$ to spin down nuclei.

\section{BCA assay-determination of protein content}

To determine the protein concentration of cell lysates Bicinchoninic acid (BCA) containing protein assay was applied. BCA reacts with $\mathrm{Cu}^{1+}$ ions forming a bluish chelat complex strongly absorbing light at $562 \mathrm{~nm} . \mathrm{Cu}^{2+}$ ions added to a protein solution are reduced to $\mathrm{Cu}^{1+}$ ions and present BCA directly develops a bluish colour proportional to the amount of protein present in the solution. A standard curve with known protein concentrations plotted against the light extinction at $562 \mathrm{~nm}$ can then be used to estimate the protein amount in a solution of unknown concentration. For the generation of the standard curve Bovine Serum albumin was used (BSA). All working solutions including BSA were taken from a BCA Protein Assay Reagent kit (Thermo scientific). The concentrations of all lysates were measured accordingly and adjusted so that all lysates had the same concentration in the end by diluting higher concentrated lysates with lysis buffer.

\section{SDS Polyacrylamide Gel-electrophoresis (SDS-PAGE)}

Proteins were separated according to their sizes by SDS-PAGE using 10-12\% NuPAGE BisTris gradient gels (Invitrogen). Protein-containing solutions were mixed with reducing SDSPAGE sample buffer including $100 \mathrm{mM}$ Tecep ${ }^{\circledR}$ Bond-Breaker to reduce disulfide bonds at $70^{\circ} \mathrm{C}$ for $10 \mathrm{~min}$. The proteins were loaded onto a polyacrylamide gel submerged in $1 \times$ MOPS or $1 \times$ MES running buffer and an electric current was applied across the gel $(80 \mathrm{~V}$ for $7 \mathrm{~min}, 180 \mathrm{~V}$ for $55 \mathrm{~min}$ ). The overall negatively charged proteins migrated to the anode in a size-dependent manner. Charge dependence can be neglected since SDS provides proteins with a very high negative total charge overriding intrinsic charges. The smaller the protein, the faster it migrates through the gel. By simultaneously applying a protein size marker, the size of the applied proteins was estimated. 


\section{Western Blot}

Western Blot technique allows protein visualisation of one specific protein within a protein mixture by antibody detection. For that purpose, the proteins were separated by SDS-PAGE and then transferred to nitrocellulose membranes by the electro-transfer method by Towbin (Towbin et al. 1979) using the Novex-blotting METHODS 43 system at $28 \mathrm{~V}$ for $135 \mathrm{~min}$. To minimise background binding of antibodies to the membranes they were usually incubated in Blocking Milk for $1 \mathrm{~h}$ at room temperature. Subsequently, the respective primary antibodies in PBS/Tween + milk + Azide were applied for $1 \mathrm{~h}$ or over night depending on the quality of the individual antibody. After washing 3 times for $10 \mathrm{~min}$ each in PBS/Tween, the blots were incubated with HRP-conjugated isotype-specific secondary antibodies diluted 1:20000 in PBS/Tween for 1-2h. Again the blots were washed thoroughly three times as before and then developed by enhanced chemoluminescence (Western lightning for recombinant protein expression analysis and $\beta$-actin, Dura for analysis of all other endogenous proteins) following the manufacturer's protocol (Amersham Pharmacia Biotech, Uppsala, Sweden).

\section{Re-cycling Western Blots for incubation with another antibody}

In order to remove antibodies from nitrocellulose membranes they were incubated in "Stripping Buffer" (50 mM glycine $\mathrm{HCl} \mathrm{pH} \mathrm{2.3)} \mathrm{for} 20 \mathrm{~min}$ at room temperature. The decrease in $\mathrm{pH}$ changes protein confirmation changing binding epitopes in a manner that antibodies can no longer bind. Subsequently, blots were washed 3 times for $5 \mathrm{~min}$ in PBS/Tween and subjected again to Blocking milk containing Azide which destroys remaining HRP.

\section{Staining for Flow Cytometry}

\section{TRAIL-R1/2 surface staining for Flow Cytometry}

Expression of surface molecules can be detected by either direct staining of the respective molecules with fluorescently labelled antibodies or use of secondary and even tertiary staining steps to enhance the signal. Positively stained cells can then be discriminated from negatively stained cells by their laser scattering profile. 
Ovarian carcinoma cells seeded into 12-well test plates over night, were then treated with the respective agents (chemotherapeutics, inhibitors and medium) for $24 \mathrm{~h}$ and then detached from the plates by $1 \times$ trypsin. After washing with PBS they were incubated with monoclonal antibodies against the two apoptosis inducing TRAIL-R1 and TRAIL-R2 $(5 \mu \mathrm{g} / \mathrm{ml})$ for 30 min on ice. One sample was incubated with control mIgG1 to control for unspecific binding of the isotype to the cell surface. Incubation with primary antibody was followed by washing 3 times with FACS buffer $(1 \times \mathrm{PBS}$ with $3 \%$ FCS $)$ and incubation with biotinylated secondary goat anti-mouse antibodies (Southern Biotechnology Associates) for $20 \mathrm{~min}$ on ice after washing again 3 times the cells were incubated in Streptavidin-PE (Pharmingen, Hamburg, Germany) for another 20 min on ice and washed again. Finally, the cells were resuspended in PI solution and analysed by flow cytometry. As PI stained cells having lost membrane integrity, non-viable cell numbers could be determined and dead cells excluded from the analyses. PI positive cells were detected in the red channel (FL-3) and PE positive and thereby TRAIL-R1 or TRAIL-R2 positive cells in the orange channel (FL-2). None of the two emission spectra interfered with the other compensation was therefore not necessary.

\section{Staining for Flow Cytometry with directly fluorescently labelled primary antibodies}

For staining of surface molecules with directly fluorescently labelled primary antibodies (CD14-FITC, CD45-APC, EpCAM-FITC) $10 \times 10^{4}$ primary ovarian cancer cells were incubated with a combination of either CD14 and CD45 or EpCAM and CD45. CD45 and CD14 were diluted 1:200, whereas EpCAM was used at a concentration of 1:10. Following incubation with the primary antibody for $30 \mathrm{~min}$. Cells were washed three times with FACS buffer and could then be analysed at the FACS. No compensation was necessary. 


\subsubsection{Isolation of primary ovarian cancer cells from ascitic fluid}

Generally, 1L of ascitic fluid was received from the nearby hospital. Prior to the first withdrawal of ascites an approval of the local ethic comitee was obtained (ethics number: 05/Q0406/178).

The volume was distributed to four $250 \mathrm{ml}$ tubes and centrifuged at $1400 \mathrm{rpm}$ for 10 minutes at $4^{\circ} \mathrm{C}$. Each pellet $(0.5-5 \mathrm{ml})$ was taken up in 5-10 $\mathrm{ml}$ ascites supernatant (SN). For Ficoll gradient centrifugation usually one ficoll gradient was taken for each $250 \mathrm{ml}$ of ascitic fluid. Depending on the purity of the pellet after the ficoll gradient centrifugation, a red blood cell lysis had to be performed. All pellets were transferred into one $50 \mathrm{ml}$ Falcon tube and were then taken up in an appropriate volume of $1 \times$ Red blood cell lysis buffer (see manufacturer's instructions; eBioscience Ltd) and incubated for $5 \mathrm{~min}$. The reaction was stopped with 5 times as much medium and centrifuged at $1400 \mathrm{rpm}$ for $3 \mathrm{~min}$. Red blood cell lysis usually cleared the pellet from all the remaining erythrocytes. The pellet was then taken up in an appropriate volume of prewarmed RPMI medium. Cells were counted and one aliquot of $2 \times 10^{5}$ cells was taken aside for flow cytometry staining.

Cells were analysed using flow cytometry and only if the ascitic fluid contained EpCAM positive cells it was proceeded to the next step of purification.

Before the first purification step with CD45-Dynabeads a so called „Fc-block“ was performed on all cells, in order to reduce unspecific binding of Fc-receptors to the CD45-Dynabeads.

$1 \times 10^{6}$ cells were incubated in a 1:100 dilution of „Fc-block“ with FACS-buffer for 5 min. They were subsequently centrifuged at $1400 \mathrm{rpm}$ for $3 \mathrm{~min}$. and taken up in medium.

CD45 Dynabeads isolation was performed according to the manufacturers instructions.

Briefly, $1 \times 10^{7}$ primary cells were transferred to a 1,5 Eppendorf tube and incubated on a rotating wheel with $100 \mu \mathrm{l}$ of washed CD45-Dynabeads in $1 \mathrm{ml}$ medium for 30 minutes at $4^{\circ} \mathrm{C}$. After incubation, the Eppendorf tube was placed on a magnet (DynaMag ${ }^{\mathrm{TM}}-2$ ) and the $\mathrm{SN}$, containing all CD45 negative cells, was carefully transferred to a clean 1,5 Eppendorf tube. CD45-Dynabeads were washed 3 times, according to the manufacturer's instructions.

CD45-positive and negative cells were counted and $2 \times 10^{5}$ of each population was taken aside for flow cytometry staining.

The CD45-negative population was now subjected to a second round of purification and 5 $\times 10^{5}$ CD45-negative cells were incubated with $25 \mu$ of washed EpCAM-Dynabeads for 35 
minutes at $4^{\circ} \mathrm{C}$ on a rotating wheel. After incubation the cells were washed as described before and taken up in an appropriate volume of medium. They were now available for further downstream analysis and could be seeded into different test plates as described above.

\begin{tabular}{|c|c|c|}
\hline Patient number & Chemotherapy pretreatment & Figure \\
\hline Patient 1 & yes & Figure 23 \\
\hline Patient 5 & yes & Figure 24 \\
\hline Patient 6 & yes & Figure 23 \\
\hline Patient 7 & yes & Figure 23 and 24 \\
\hline Patient 8 & yes & Figure 26 \\
\hline
\end{tabular}

\subsubsection{Production of moTAP-TRAIL}

The Modified Tandem Affinity Purification (moTAP)-tagged (Haas et al. 2009) active version of TRAIL comprises the extracellular domain (ECD) of TRAIL and a Modified Tandem Affinity Purification tag. The moTAP tag consists of a His-tag, $3 \times$ Flag-tag, followed by a precision site and an AviTag [figure 9]. This tag allows a two-step purification resulting in reduced unspecific binding of protein and a very pure receptor signalling complex. As a first step, moTAP-TRAIL, together with the TRAIL-DISC will be immuno-precipitated via its FLAG tag by M2-anti-FLAG antibody-coupled beads. The FLAG-tag can then be cleaved off by a specific protease (precision), and the remaining TRAIL bound to TRAIL-DISC is eluted. The second purification step consists of the affinity precipitation by Streptavidin via the biotinylated Avi-tag that forms part of the moTAP-tag of TRAIL (Haas et al. 2009).

The His-tag is used for purification of the mo-TAP TRAIL, because it specifically binds to a Ni-NTA Sepharose (QIAGEN) containing column.

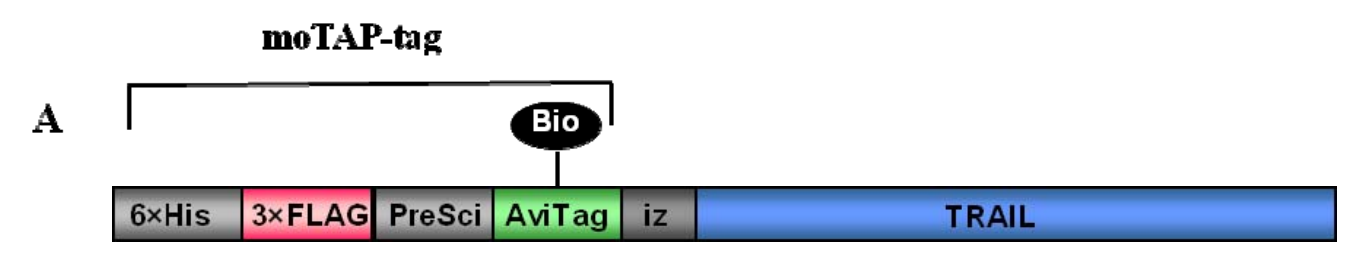

Figure 9. The moTAP-tag comprises a His-tag for Ni-NTA purification, FLAG-tag for the first immunoprecipitation and a biotinylation site for the second immuno-precipitation. 


\section{Expression and purification of moTAP-TRAIL}

The E. coli strain AVB 101 (purchased from Avidity) was used for expression of moTAP-TRAIL. The bacteria contain the biotinylating enzyme BirA in the expressionvector PaCYC (chloramphenicole resistance) and were already transformed with moTAP-TRAIL cloned into the the bacterial expression vector PQE-30 (ampicillin resistance). Both proteins are inducible by IPTG but have a low constitutive expression already since AVB 101 lacks the lac repressor. To establish expression of moTAP-TRAIL under optimal conditions different temperatures, duration of expression and amounts of IPTG have been tested (data not shown). Finally, $4 \mathrm{~L}$ of bacterial culture were inoculated and incubated until the $\mathrm{OD}_{600 \mathrm{~nm}}$ had reached 0,6. Subsequently, IPTG [100 iM] and Biotin [50 iM] were added to induce the production of biotinylated moTAP-TRAIL followed by incubation overnight at $18^{\circ} \mathrm{C}$. On the next day, cells were harvested by centrifugation at $4600 \mathrm{rpm}$ for $30 \mathrm{~min}$ at $4{ }^{\circ} \mathrm{C}$ and bacteria were lysed using Bacteria lysis buffer supplemented with lysozyme [50 ìg/ml] and benzonase [5 U/ml]. Three freeze and thaw cycles, in liquid nitrogen and at $42^{\circ} \mathrm{C}$ as well as sonification steps (3-5 times, $30 \mathrm{sec}$, duty cycle 30, output control 40) were performed to further disrupt the bacteria. To pellet still unlysed bacteria and cell debris, the solution was first centrifuged at $4600 \mathrm{rpm}$ for $30 \mathrm{~min}$ at $4^{\circ} \mathrm{C}$ and afterwards at $15.000 \mathrm{rpm}$ for $30 \mathrm{~min}$ at $4^{\circ} \mathrm{C}$ to remove the inclusion bodies. The supernatant was then filtered using $0.45 \mathrm{ìm}$ syringe filters and applied to a Ni-NTA Sepharose (QIAGEN) containing column (100 ml volume).

The Ni-NTA Sepharose (QIAGEN) containing column was equilibrated with bacteria lysis buffer ( $2 \mathrm{mM} \beta$-Mercaptoethanol w/o protease inhibitors inhibitors). The filtered lysate was applied and followed by two washing steps with bacteria lysis buffer and column wash buffer. All flow troughs were collected for later analysis. Elution of moTAP-TRAIL was performed using Imidazole and 30 fractions à $10 \mathrm{ml}$ were collected. A maximum flow rate of $3 \mathrm{ml} / \mathrm{min}$ and the maximum pressure limit of $0.3 \mathrm{mpa}$ was never exceeded in order to avoid damage of the column material. After analysis of the elution fractions via SDS PAGE and western blot for purification analysis moTAP-TRAIL containing fractions were pooled (here fractions 916) and dialysed. The protein was firstly dialysed in 5L of maintenance buffer w/o L-Arginin over night and then in 3L of maintenance buffer with L-Arginin over night. Aliquots of purified moTAP-TRAIL were stored at $-80^{\circ} \mathrm{C}$.

As shown in figure 10A moTAP-TRAIL specifically binds to the column and does not elute during the two washing steps. To ensure, that moTAP-TRAIL is expressed in an intact form 
with all necessary tags present, these tags were analysed by WB using an $\alpha$-FLAG-tag M2 Antibody, $\alpha$-His Antibodies and Streptavidin-HRP for the detection of the biotinylated AviTag of moTAP-TRAIL. In order to estimate the concentration of the newly purified moTAP-TRAIL it was diluted and subjected to WB analysis [figure 10B] compared to different known concentrations of iz-TRAIL, thus estimating its concentration. The intensity of the band of 25ng iz-TRAIL was comparable to a 1:10 diluted mo-TAP TRAIL on that Blot, resulting in an estimated concentration of $250 \mathrm{ng} / \mathrm{ml} \mathrm{moTAP-TRAIL.}$

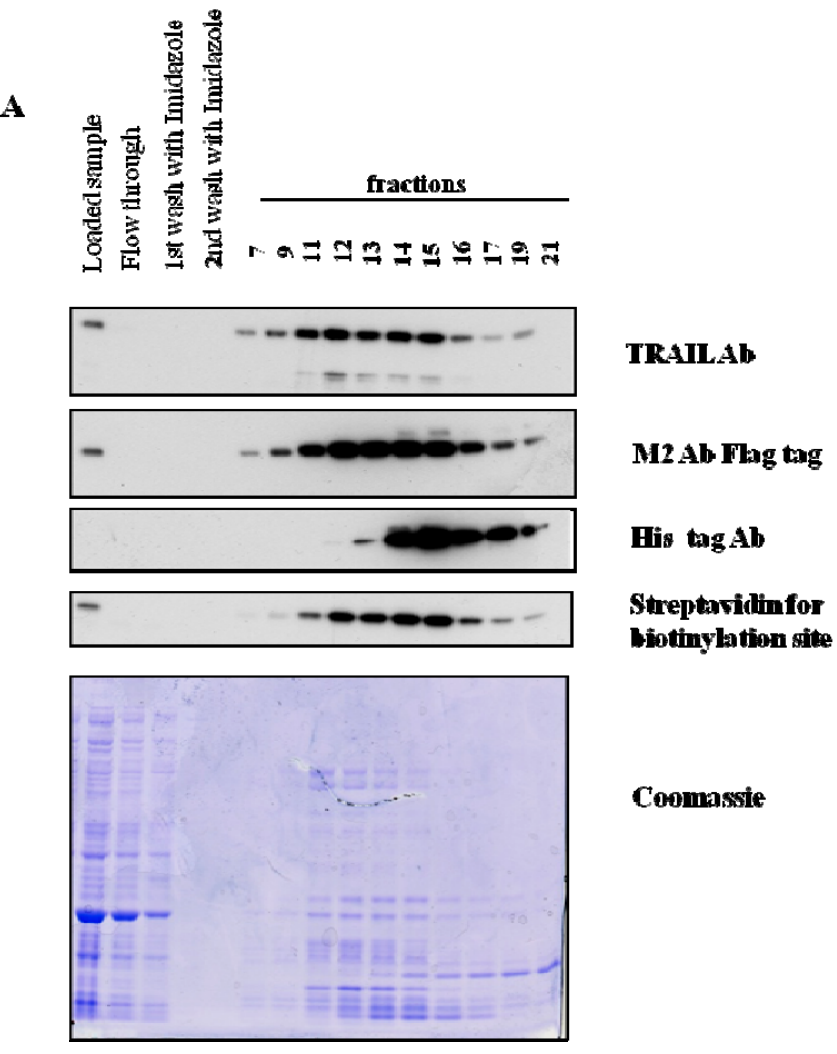

Figure 10: Production and Purification of recombinant Human moTAP-TRAIL: B. The initially loaded sample onto Ni-NTA column, Flow through, the two wash steps and the different elution fractions were analysed by WB for the expression of the distinct tags of moTAP-TRAIL $\mathbf{C}$. The concentration of the moTAP-TRAIL was estimated by comparing different dilutions of moTAP-TRAIL with defined concentrations of iz-TRAIL. Estimated concentration of moTAP-TRAIL 250ng/ml. 


\section{Testing Functionality of purified moTAP-TRAIL}

Following purification the functionality of moTAP-TRAIL needed to be tested. It was determined, whether the newly produced moTAP-TRAIL can effectively induce apoptosis and be used to immune-precipitate the TRAIL-DISC.

HCT-116 cells were used for the following experiments as they are known to be sensitive to TRAIL-induced apoptosis (Ozoren and El-Deiry 2002).

HCT-116 cells were incubated with increasing amounts of moTAP-TRAIL and were efficiently killed at a concentration of only $\sim 13 \mathrm{ng} / \mathrm{ml}$ moTAP-TRAIL [figure 11A].

In order to further determine the induction of apoptosis as a TRAIL-specific event, moTAP-TRAIL was pre-incubated with TRAIL-R2 Fc protein and then added to the cells. This lead to blockage of $\sim 80 \%$ of apoptosis induction by TRAIL [figure 11B]. Hence, potentially toxic impurities contained in the moTAP-TRAIL preparation could widely be excluded.

In order to test whether the DISC could be immuno-precipitated using moTAP-TRAIL, HCT-116 cells were seeded into cell culture petri dishes $\left(50 \mathrm{~cm}^{2}\right)$ at $1 \times 10^{6}$ cells per dish one day in advance. MoTAP-TRAIL was added at an estimated concentration of $1 \mu \mathrm{g} / \mathrm{ml}$ in $10 \mathrm{ml}$ medium to the cells after the remaining medium was removed. Stimulation was stopped by removing moTAP-TRAIL containing medium and washing cells in ice-cold PBS after 0 (unsitmulated), 10 and15 min. The PBS was removed and cells were scraped off the dishes and transferred to a falcon tube. After spinning down the cells they were resuspended in lysis buffer (30 mM Tris- $\mathrm{HCl} \mathrm{pH} \quad 7.5,150 \mathrm{mM} \mathrm{NaCl}, 10 \%$ glycerol $1 \%$ Triton X-100) supplemented with Complete ${ }^{\mathrm{TM}}$ protease inhibitors (Roche Diagnostics, Mannheim, Germany) and incubated for $45 \mathrm{~min}$ on ice. The lysates were then incubated with $60 \mu 1 \mathrm{M} 2$ beads diluted at a ratio of 1:3 with Sepharose CL-4B beads in a $1.5 \mathrm{ml}$ Eppendorf tube at $4^{\circ} \mathrm{C}$ overnight to allow binding of the proteins via their FLAG-tag to the M2 antibodies on the surface of the beads. To the zero timepoint lysate $3 \mu 1$ of moTAP-TRAIL was added to make sure that components of the DISC only assemble at the intact cell's membrane and not spontaneously and unspecifically in the lysates. After overnight incubation the tubes were centrifuged and a sample was taken from the supernatants for later analysis of proper binding of all moTAP-TRAIL molecules to the beads. The beads were washed five times and then resuspended in $20 \mu 12 \times$ reducing sample buffer. After heating the samples to $70^{\circ} \mathrm{C}$ for 10 min they were subjected to SDS-PAGE and Western Blot subsequently [figure 11C]. 
A

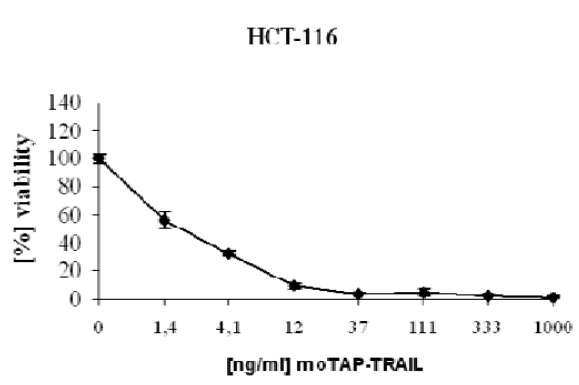

B

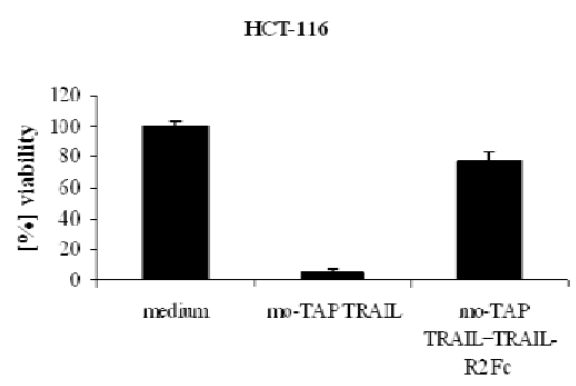

C
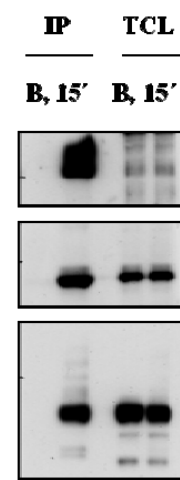

TRAIL-R2

46

FADD

28

Caspase-8 $55 / 53$

Caspase-8 43/4t

cleaved

CFLIPL

Actin $\quad 43$

Figure 11: Functionality testing of recombinant Human moTAP-TRAIL: A. HCT-116 cells were treated with moTAP-TRAIL for $24 \mathrm{~h}$ at the indicated concentrations. Cell viability was determined by MTT assay. B. HCT-116 cells were subjected to treatment with $250 \mathrm{ng} / \mathrm{ml}$ moTAP-TRAIL in the presence or absence of 2,5 $\mu \mathrm{g} / \mathrm{ml}$ TRAIL-R2-Fc protein for 24h. moTAP-TRAIL and TRAIL-R2-Fc protein were pre-incubated for 30 minutes to allow for binding before applied to cells. C. HCT-116 cells were either stimulated with $1 \mu \mathrm{g} / \mathrm{ml}$ moTAP-TRAIL for 15 minutes or left un-stimulated ( $\mathrm{B}=$ Beads only). Cell lysates were prepared and the TRAILreceptor complex was immuno-precipitated over night with M2 beads that bind to the Flag-tag. $30 \mu 1$ of lysates were removed before immuno-precipitation to control for equal expression of protein levels (Total cell lysates $=\mathrm{TCL})$. Results were generated from triplicate values. 
4. Results 


\section{Results}

\subsection{Assessment of sensitivity of ten different ovarian cancer cell lines to TRAIL-induced apoptosis}

Ten different well established ovarian carcinoma cell lines of distinct histopathological subtypes were assessed for their sensitivity to TRAIL induced apoptosis.

The majority of cell lines are derived from serous ovarian cancer including HEY and SKOV3 cells, as well as PEO-1 and PEA-1 and their isogenic matched pairs PEO-4 and PEA-2 which are Cisplatin resistant [figure 12A]. Furthermore, an endometrioid ovarian cancer cell line TOV-112D and the clear cell ovarian cancer cell line, KK, were tested [figure 12B]. IGROV-1 and IOSE-80 are undifferenciated ovarian cancer cell lines, which can not be integrated into any of the major histopathological groups [figure 12C]. The form of TRAIL that was used for the sensitivity screen is a very active form of TRAIL tagged with an isoleucine zipper (iz), (the iz leads to trimerisation of the ligand and subsequent increased oligomerisation of TRAIL-receptors), generated in our lab (Ganten et al. 2006). Four cell lines (PEO-1, PEO-4, IOSE80 and HEY) were sensitive to TRAIL-induced apoptosis, as defined by loss of viability by at least $50 \%$ after treatment with $1 \mu \mathrm{g} / \mathrm{ml}$ iz-TRAIL [figure 12A,C]. PEA-1, SKOV-3, IGROV-1, KK and TOV-112D cells exhibited complete resistance to TRAIL, whereas PEA-2 cells were partially sensitive. Therefore, we conclude that there is no correlation between TRAIL-sensitivity and histological subtype in this panel of ovarian cancer cell lines. 
$\mathbf{A}$

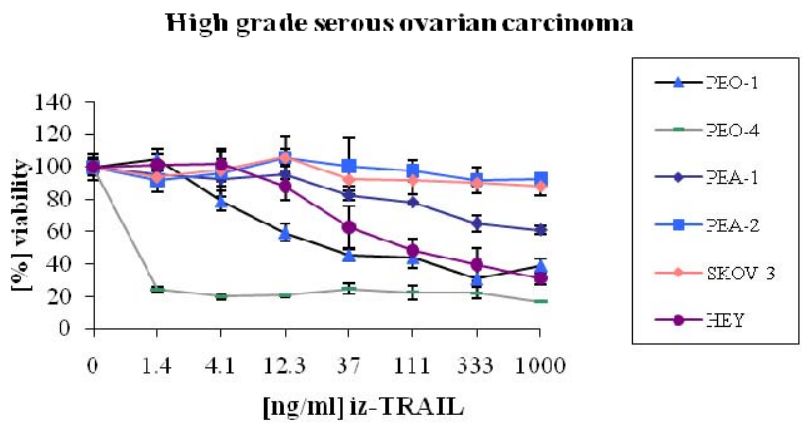

B

Endometrioid and Clear cell ovarian carcinoma

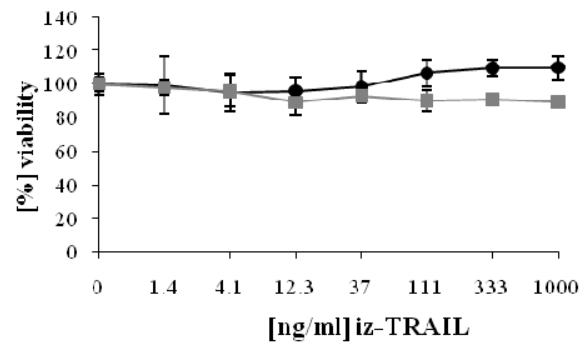

C

Undifferentciated ovarian carcinoma

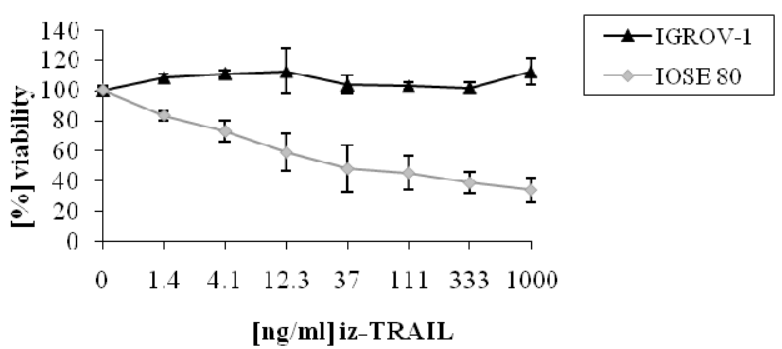

Figure 12: Sensitivity of different cell lines to TRAIL induced apoptosis. Cells were incubated with increasing concentrations of iz-TRAIL for 24h. A. Sensitivity of PEO-1,PEO-4, PEA-1, PEA-2, SKOV-3, HEY to TRAIL-induced apoptosis. B. Sensitivity of TOV-112D and KK to TRAIL-induced apoptosis. C. Sensitivity of IGROV-1, IOSE 80 to TRAIL-induced apoptosis. Cell viability was measured by MTT assay. Error bars represent standard deviation. Experiments were performed in triplicates and one representative of at least two independently performed experiments is shown.

\subsection{Toxicity titrations for potential sensitising agents}

Three cell lines (TOV-112D, KK, PEA-1), each from one distinct histopathological subtype, all displaying resistance to TRAIL-induced apoptosis, were chosen for further sensitisation experiments. In addition, PEA-2 cells were chosen in order to detect possible differences between cisplatin-resistant and -sensitive ovarian cancer cells to TRAIL-induced apoptosis.

To reduce possible unwanted effects, subtoxic doses of the sensitising agents had to be determined for each cell line [figure 13A-C].

The sensitising agents used were Bortezomib (PS-341, Velcade ${ }^{\circledR}$, Proteasome-inhibitor), PIK75 (PI3K-alpha inhibitor) and Smac59 or Smac83 in later experiments, (IAP-antagonists). 
Smac59/83 caused only slight toxicity, whereas PIK75 exhibited toxic effects to TOV-112D cells and PEA-2 cells, but leaving the other two cell lines largely unaffected.

Bortezomib also showed a higher toxicity in TOV-112D and PEA-2 cells, than in KK and PEA-1 cells.

A

Bortezomil

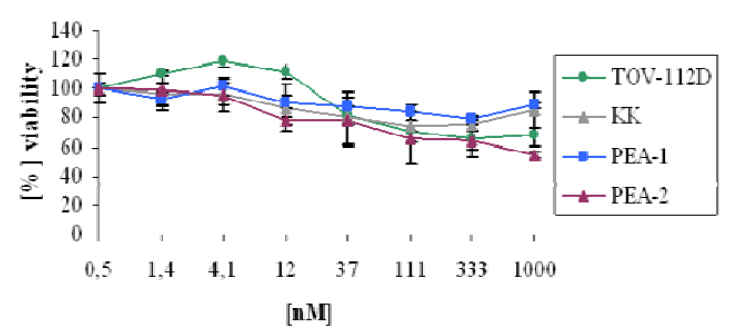

C

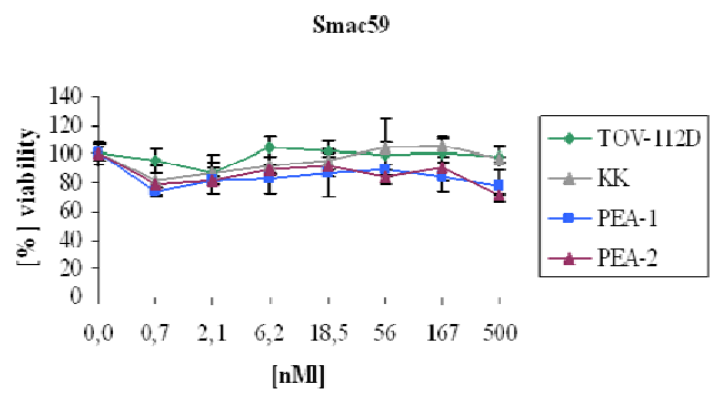

$\mathbf{B}$

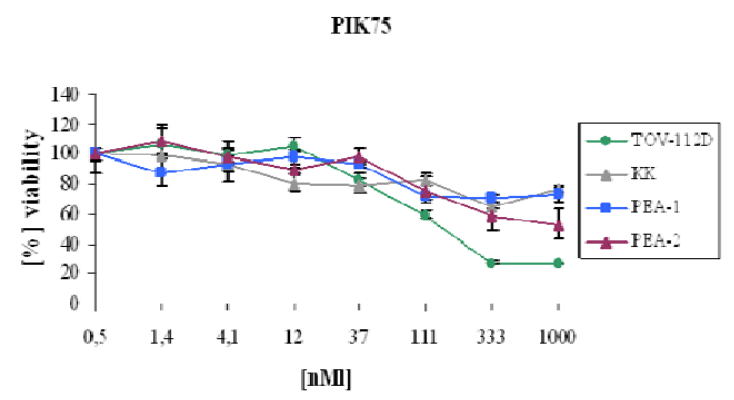

Figure 13: Dose response curves for Bortezomib, PIK75 and Smac 59 in TOV-112D, KK, PEA-1 and PEA-2 ovarian cancer cells. Cells were incubated with increasing concentrations of Bortezomib, PIK75 and Smac59 for 24h. Cell viability was measured by MTT assay. A-C Toxicity was titrated for TOV-112D, KK, PEA-1 and PEA-2. Error bars represent standard deviation. Experiments were performed in triplicates and one representative of at least two independently performed experiments is shown.

\subsection{Bortezomib sensitises KK, PEA-1 and PEA-2 cells but not TOV- 112D cells to TRAIL-induced cell death}

In order to sensitise TRAIL-resistant cells to TRAIL-induced apoptosis, they were co-incubated with increasing amounts of TRAIL (highest concentration $1 \mu \mathrm{g} / \mathrm{ml}$ ) and a subtoxic dose of Bortezomib as determined in figure 13. 
From all the different sensitising drugs tested, only Bortezomib was able to sensitise KK cells very effectively to TRAIL-mediated apoptosis, whereas TOV-112D cells remained resistant [figure 14A,B]. The reduced viablity in TOV-112D cells was solely due to Bortezomib alone. PEA-1 and PEA-2 cells could also be sensitised very efficiently by Bortezomib [figure 14C, D].

A

KK

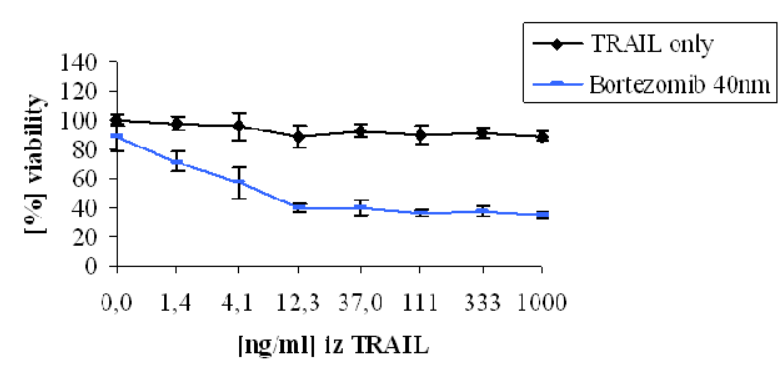

C

PEA-2

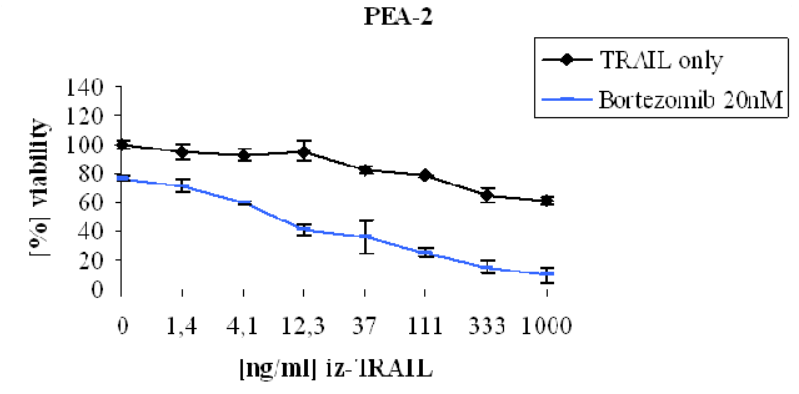

B

PEA-1

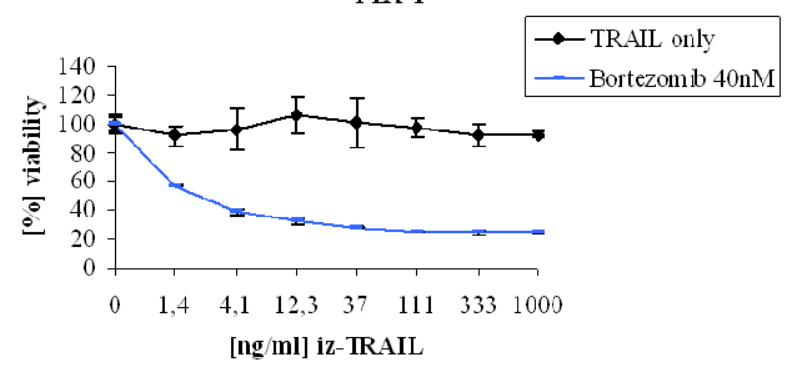

D

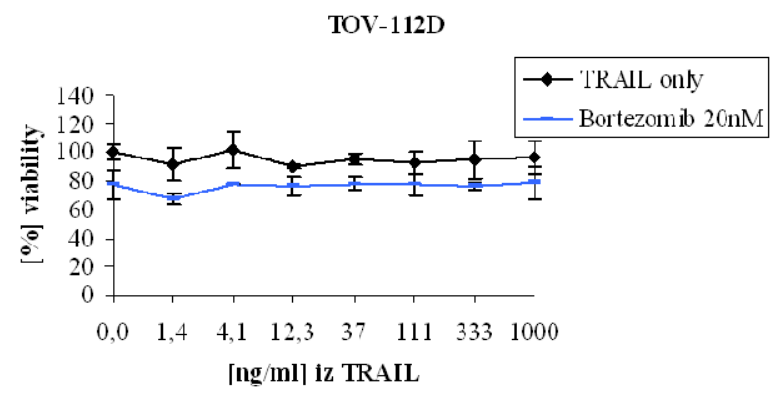

Figure 14: KK, PEA-1 and PEA-2 cells can be sensitised to TRAIL-induced apoptosis with Bortezomib. A+B. KK and PEA-1 cells were co- incubated with 40nM Bortezomib and $1 \mu \mathrm{g} / \mathrm{ml}$ iz-TRAIL. C+D. PEA-2 and TOV-112D cells were incubated with 20nM Bortezomib + and increasing content of iz-TRAIL. Cell viability was measured by MTT assay afther 24h. Experiments were performed in triplicates and one representative of at least two independently performed experiments is shown. 


\subsection{Bortezomib-mediated sensitisation to TRAIL specifically induces apoptosis}

To test whether the cells die of apoptosis when treated with TRAIL in combination with the different drugs, they were analysed by Flow Cytometry for hypo-diploid DNA content according to Nicoletti et al. 1991 (Nicoletti et al. 1991).

This analysis revealed that Bortezomib-mediated sensitisation to TRAIL led to apoptosis, as determined by increased amounts of cells with sub-diploid DNA-content [figure 15A-C]. Additionally, the effect of Cisplatin as a first-line chemotherapy agent applied in ovarian cancer was determined. Surprisingly, KK and PEA-1 cells exhibited complete resistance to Cisplatin as a single agent. Only PEA-2 cells that are described to be Cisplatin-sensitive, underwent apoptosis upon Cisplatin treatment.

$\Lambda$

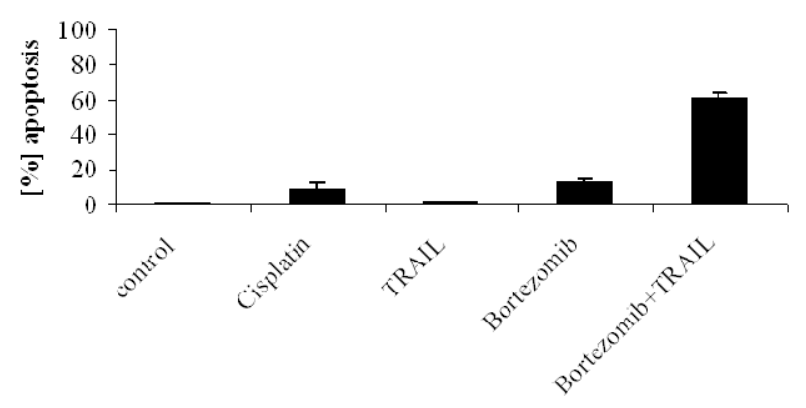

C.

PEA-2

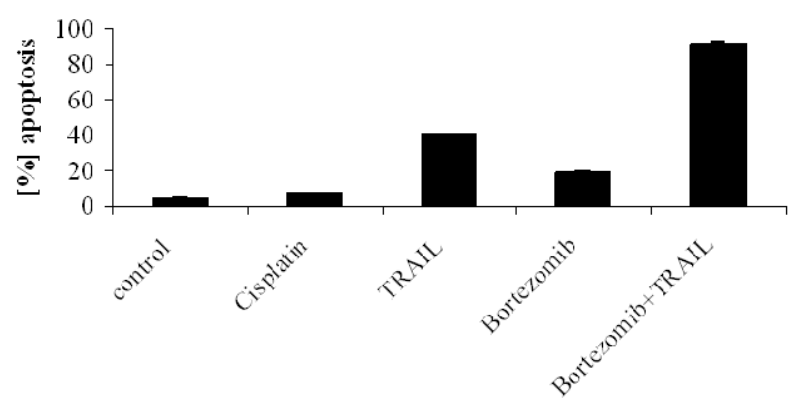

B

PEA-1

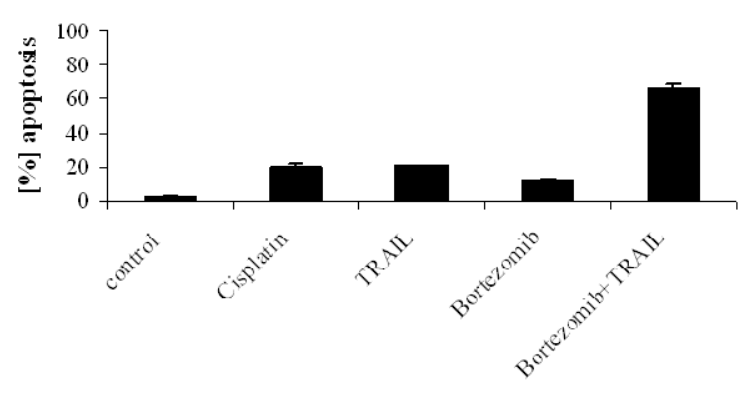

Figure 15: Bortezomib-mediated sensitisation to TRAIL specifically induces apoptosis in KK, PEA-1 and PEA-2 cells.

A+B. KK and PEA-1 cells were treated with $1 \mu \mathrm{g} / \mathrm{ml}$ Cisplatin or co-incubated with $40 \mathrm{nM}$ Bortezomib and $1 \mu \mathrm{g} / \mathrm{ml}$ iz-TRAIL G. PEA-2 cells were treated with $1 \mu \mathrm{g} / \mathrm{ml}$ Cisplatin or co-incubated with $20 \mathrm{nM}$ Bortezomib and $1 \mu \mathrm{g} / \mathrm{ml}$ iz-TRAIL. E-G. Apoptosis was examined by subdiploid DNA content after $24 \mathrm{~h}$. Values are mean +/- standard deviation (SD) of two independent experiments, each performed in duplicates. 


\subsection{Smac83 and PIK75 sensitise PEA-1 cells and PEA-2 cells to TRAIL-induced apoptosis}

As described for Bortezomib, the cells were co-treated with subtoxic doses of Smac83 or PIK75 and TRAIL for 24h. Apoptosis was then analysed by Flow Cytometry.

Especially PEA-2 cells but also PEA-1 cells could potently be sensitised to TRAIL-induced apoptosis by both sensitising drugs [figure 16A, B]. KK cells could also be sensitised by both agents [figure 16C]. The apoptotic effect was however, less pronounced when compared to PEA-1 and PEA-2, we decided to only use PEA-1 and PEA-2 cells for further analysis.

A

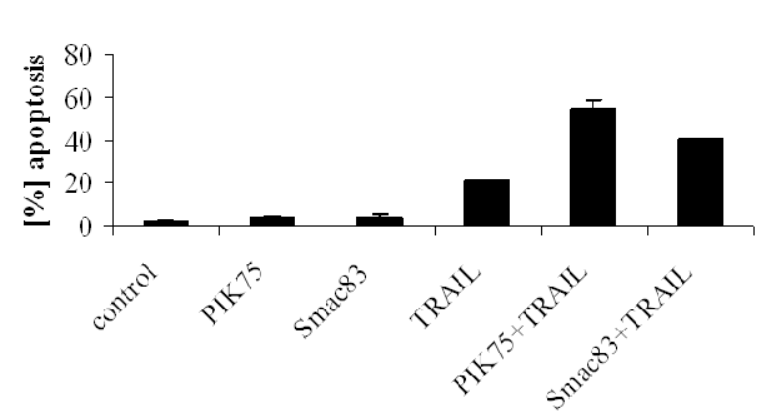

C

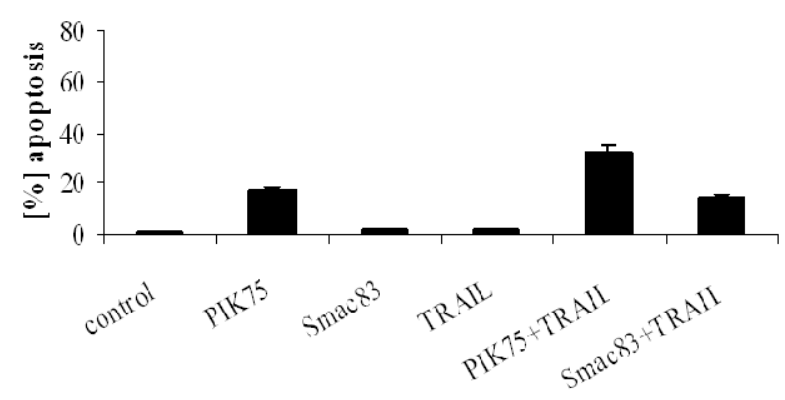

KK
B PEA-2

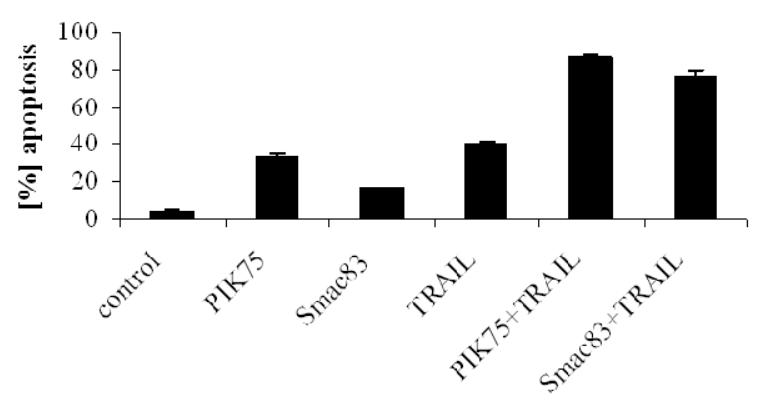

Figure 16: PEA-1, PEA-2 and KK cells can be sensitised to TRAIL-induced apoptosis with Smac83 and PIK75. A-C. KK, PEA-1 and PEA-2 cells were treated with $1 \mu \mathrm{g} / \mathrm{ml}$ iz-TRAIL and/or 100nM Smac83 or PIK75, respectively. The number of apoptotic cells was determined by subdiploid DNA content. Values are mean $+/-$ standard deviation (SD) of three independent experiments, each performed in duplicates. 


\subsection{Cell death induction can be efficiently blocked by caspase- inhibitors but not by Necrostatin-1, a RIP-1 kinase activity inhibitor}

In order to further determine the specificity of the sensitising agents plus TRAIL to induce apoptosis and no other form of cell death, caspase inhibitors were applied. Caspase inhibitors irreversibly bind to the catalytic site of caspase proteases and thereby inhibit apoptosis. QVD-Oph is a broad-spectrum caspase inhibitor that has shown to be a very potent apoptosis inhibitor with low toxicity (Caserta et al. 2003) .

In contrast, Necrostatin-1 can inhibit RIP-1, the key upstream kinase that is involved in the activation of the necroptotic pathway. The term necroptosis has been recently established for a newly discovered type of programmed necrosis that depends on the activation of RIP-1 (Degterev et al. 2008; Hitomi et al. 2008).

The cells were pre-treated with either Necrostain-1 or Q-VD-Oph for one hour to allow for effective inhibition of RIP-1 and caspases, respectively. Then they were treated with TRAIL plus sensitising agent for 24 hours. The results show that Q-VD-Oph effectively blocked apoptosis in PEA-1, PEA-2 and KK cells [figure 17A-C]. RIP-1 inhibition by Necrostain-1 on the other hand was not able to block sensitisation.

Taken together, it could be shown that Bortezomib, Smac83 and PIK75 sensitise PEA-1, PEA-2 and KK cells specifically to TRAIL-mediated apoptosis. 


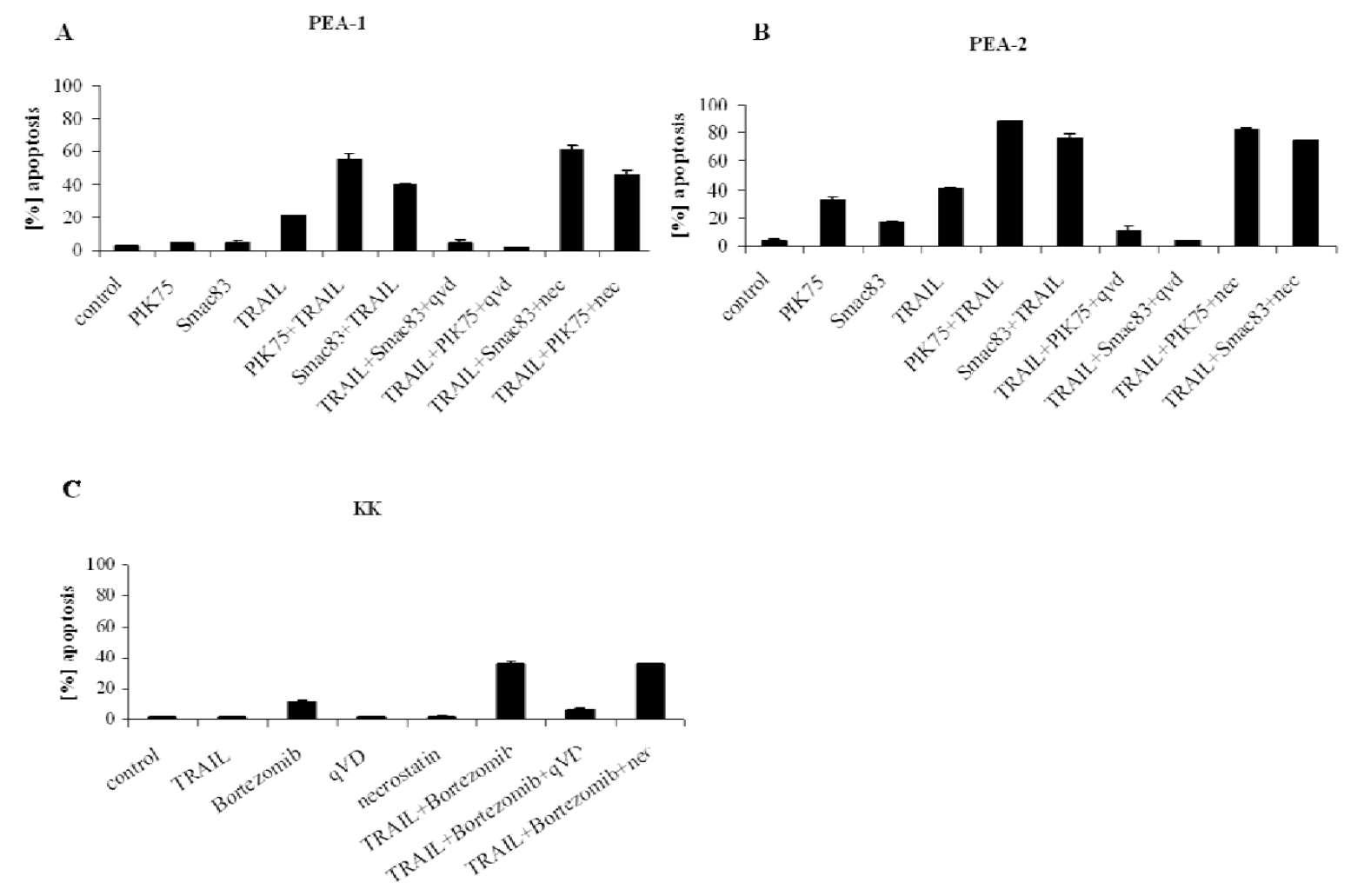

Figure 17: TRAIL-induced apoptosis can be blocked with QVD-OPh or zVAD-fmk but not with necrostatin-1. A-C. Cells were pre-incubated with $10 \mu \mathrm{M}$ QVD-OPh and/or zVAD-fmk or 30 $\mu \mathrm{M}$ necrostatin-1 for $1 \mathrm{~h}$ and were subsequently treated with $1 \mu \mathrm{g} / \mathrm{ml}$ TRAIL plus inhibitors. (100nM PIK75, 100nM Smac83, 40nM Bortezomib). Number of apoptotic cells were determined by subdiploid DNA content. Values are mean +/- standard deviation (SD) of three independent experiments, each performed in duplicates.

\subsection{Molecular changes in the TRAIL-receptor pathway induced by small molecule inhibitors}

In order to investigate at which stage the inhibitor affects the TRAIL apoptosis pathway, Western Blot (WB) analysis of the effect of different inhibitors on known effector molecules of TRAIL-induced apoptosis was performed. Cells were treated with the sensitising agent for different times to determine the optimal condition at which anti-apoptotic and pro-apoptotic molecules are downregulated or upregulated, respectively.

In KK cells, Bortezomib treatment led to a slight but reproducible down-regulation of cFLIP $\mathrm{L}_{\mathrm{L}}$ after $24 \mathrm{~h}$ which is known to correlate with TRAIL resistance (Jonsson et al. 2003) [Figure 18.1A]. cFLIP $_{S}$ on the other hand is upregulated upon Bortezomib treatment which goes in 
line with results that have been obtained by others (Koschny et al. 2010). Furthermore, a strong down-regulation of XIAP could be detected over a timecourse of $24 \mathrm{~h}$ in KK cells. High XIAP levels, have also been reported to contribute to resistance to TRAIL-mediated apoptosis (Vogler et al. 2009).

Further inhibitors of apoptosis were examined, but no differences in cIAP-1 or cIAP-2 expression levels were detected in these total cell lysates after $24 \mathrm{~h}$. Neither caspase- 8 and caspase-3 nor Bid showed signs of activation such as cleavage fragments. This supports the data in figure 14E that there is no detectable apoptosis induction upon inhibitor treatment only. However, Bid was up-regulated in KK cells upon Bortezomib treatment, so more Bid is available for cleavage and, hence, induction of the mitochondrial amplification loop. Increased cleavage of Bid has been described as one of the effects exerted by Bortezomib (Morgillo et al. 2010). Most importantly, Bortezomib treatment causes an up-regulation of FADD and together with the cFLIP down-regulation has been reported to allow for more effective DISC formation (Koschny et al. 2007c).

As many drugs have been shown to increase the expression of TRAIL-R levels, it was investigated, whether the sensitising effect might be caused by changing the pattern of TRAIL-Rs.

When determining the expression of apoptosis-inducing TRAIL-R1 and -R2 KK cells showed low expression of TRAL-R1 and relatively high expression of TRAIL-R2 [Figure 18.1B] on their surface. Upon treatment with Bortezomib both receptors were upregulated, even though TRAIL-R1 was a lot more upregulated than TRAIL-R2 which was already expressed at higher levels under native conditions. In order to determine, whether the upregulation of TRAIL-R1 and -R2 is responsible for the sensitisation effect or whether it only correlates with it a series of "wash-kill" experiments was performed by Ganten and Haas (Ganten et al. 2004). As previously shown in our lab, sensitisation to TRAIL by Bortezomib does, however, not rely on the up-regulation of apoptosis-inducing TRAIL receptors, but rather on more efficient DISC assembly via enhanced FADD recruitment (Ganten et al. 2004). 

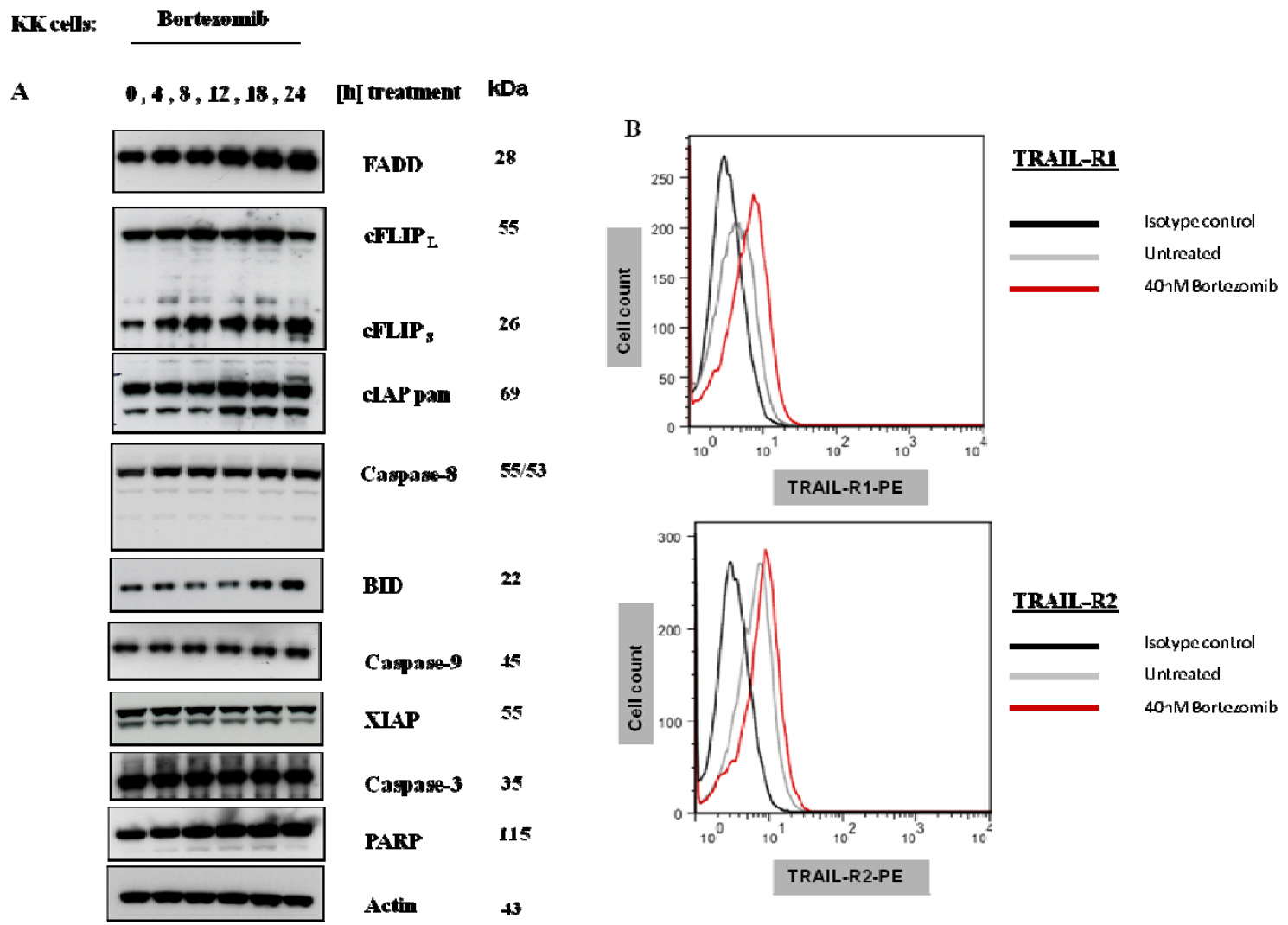

Figure 18.1: Bortezomib changes the expression pattern of different molecules along the TRAIL signalling pathway: A. $2 \times 10^{5} \mathrm{KK}$ cells were treated with $40 \mathrm{nM}$ Bortezomib for the indicated time. Cell lysates were prepared as described in chapter 3 and WB analysis was performed. B $1 \times 10^{5} \mathrm{KK}$ cells were treated with $40 \mathrm{nM}$ Bortezomib for $24 \mathrm{~h}$. Cells were subsequently stained for TRAIL-R1 and -2 and receptor expression levels were measured by Flow Cytometry as described in chapter 3 and compared to the Isotype control (mIgG1).

The WB analysis of PEA-1 and PEA-2 cells upon PIK75 or Smac83 treatment showed a very strong down-regulation of $\mathrm{cFLIP}_{\mathrm{L}}, \mathrm{cFLIP}_{\mathrm{S}}$ and cIAPs, respectively [Figure 18.2A,B].

Already after $4 \mathrm{~h}$ of PIK75 treatment in PEA-1 cells and $8 \mathrm{~h}$ in PEA-2 cells a potent downregulation of $\mathrm{cFLIP}_{\mathrm{L}}$ and $\mathrm{cFLIP}_{\mathrm{S}}$ could be detected as well as a strong downregulation of XIAP. cIAP-1/-2 showed a slight downregulation after $18-24 \mathrm{~h}$ but only in PEA-2 cells. Again, no caspase cleavage was detected upon inhibitor-only treatment. The Smac mimetics kinetic was performed with two different compounds-Smac83 and Smac59. Smac83 is an optimised version of Smac59, and the activity of both compounds needs to be compared. The comparative kinetic showed no differences between the two compounds so that in this context they can be used interchangeably. After treatment with Smac59 or Smac83 a rapid degradation of cIAP-1 and cIAP-2 could be observed. Surprisingly, eventhough cFLIPS 
markedly increased, the sensitisation effect of PEA-1 and PEA-2 cells had occurred, nevertheless.

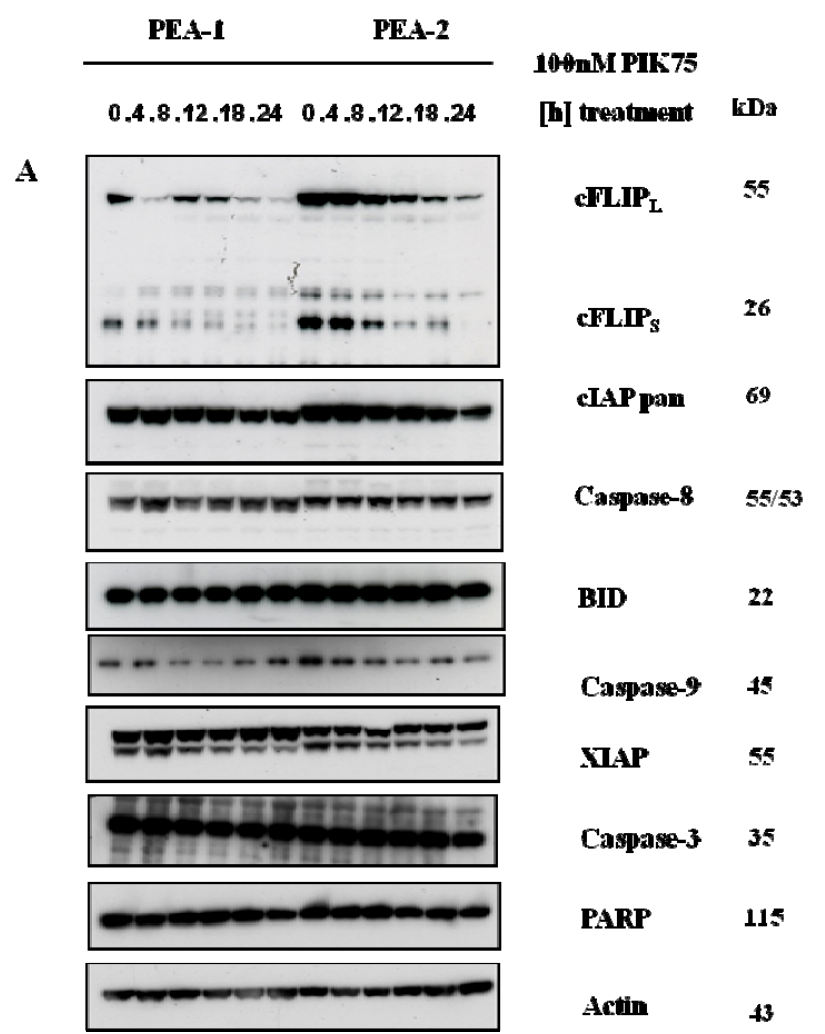

B

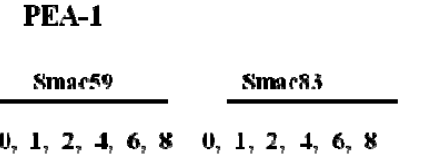

PEA-2
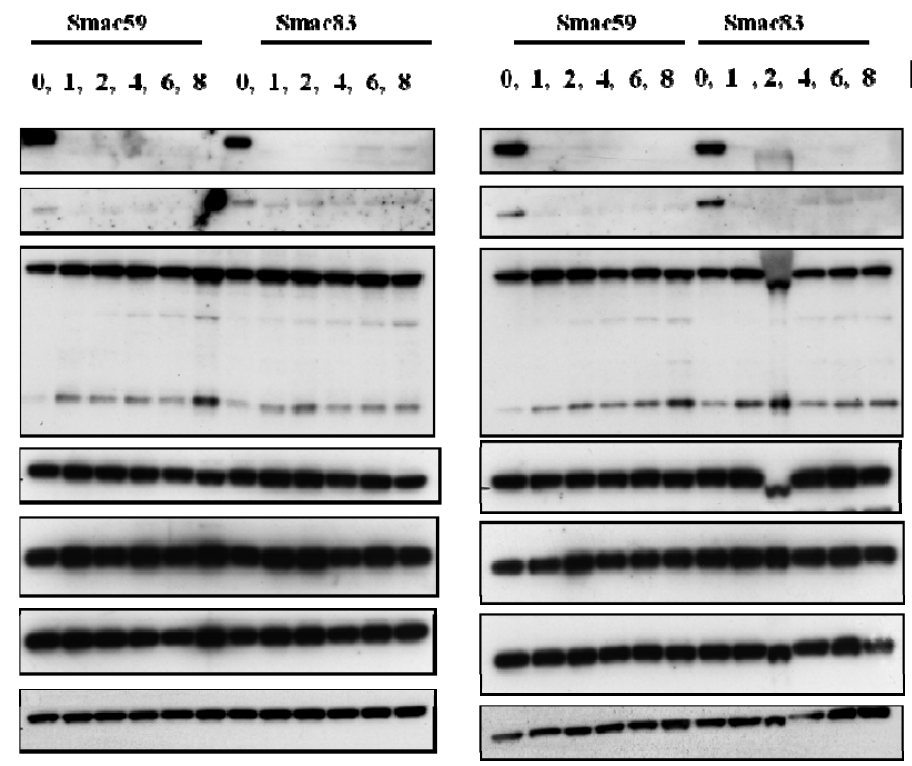

[h] Treatment kDa

cLAP-1 69

CIAP-2 $\quad 68$

cFLIP $_{\text {L }} \quad 55$

CFT.IP $\quad 26$

XIAP 55

Caspase-9 45

Caspase-3 35

Actin $\quad 4$

Figure 18.2: PIK75 and Smac83 change the expression pattern of different molecule along the TRAIL signalling pathway: A+B. $2 \times 10^{5}$ PEA-1 and PEA-2 cells were treated with $100 \mathrm{nM}$ PIK75 or Smac83/Smac59 for indicated timepoints. Cell lysates were prepared a described in chapter II and WB analvsis was performed. 


\subsection{Small molecule inhibitors allow for TRAIL-induced cleavage of caspases and cleavage of their substrates}

In addition to quantification of apoptosis, Western Blot analysis of proteins associated with the TRAIL-R pathway was performed to examine at which stage of the TRAIL-R pathway these compounds sensitise to TRAIL-induced apoptosis.

KK cells were treated with $1 \mu \mathrm{g} / \mathrm{ml}$ iz-TRAIL for different times, to determine mechanisms in apoptosis induction ranging from early events to late processes, at which the cascade is fully activated. The concentration of $1 \mu \mathrm{g} / \mathrm{ml}$ iz-TRAIL is not lethal to $\mathrm{KK}$ cells, so that they showed little to no signs of apoptotic processing, except for $\mathrm{CFLIP}_{\mathrm{L}}$ which was partially cleaved [figure 19.1]. Cells that were pre-treated with Bortezomib for 15h, a time identified by the inhibitor-only kinetic analysis in figure 18.1.A to enable FADD and cFLIP $_{S}$ upregulation, showed cleavage of cFLIP $_{\mathrm{L}}$ and caspase-8 after 30 minutes, indicating assembly of an active DISC. After 30 minutes the fully cleaved p18 fragment was generated. Full cleavage of caspase-3 (p17) and caspase-9 (p35) can be detected after 30 minutes. Caspase-3 activity was also evaluated by cleavage of its substrate PARP. Full cleavage of PARP was detected after $2 \mathrm{~h}$. The DMSO pre-treated cell population showed no PARP cleavage, not even after 6 hours iz-TRAIL stimulation. Furthermore, complete cleavage of cFLIP $_{\mathrm{L}}$ at the DISC allows for the activation of caspase-8 and induction of apoptosis in Bortezomib pre-treated cells. However, in DMSO pre-treated cells, cFLIP ${ }_{\mathrm{L}}$ cannot be cleaved completely, blocking the downstream pathway of apoptosis. Degradation of Bid and cleavage of caspase-9 can only be detected in KK cells pre-treated with Bortezomib. 

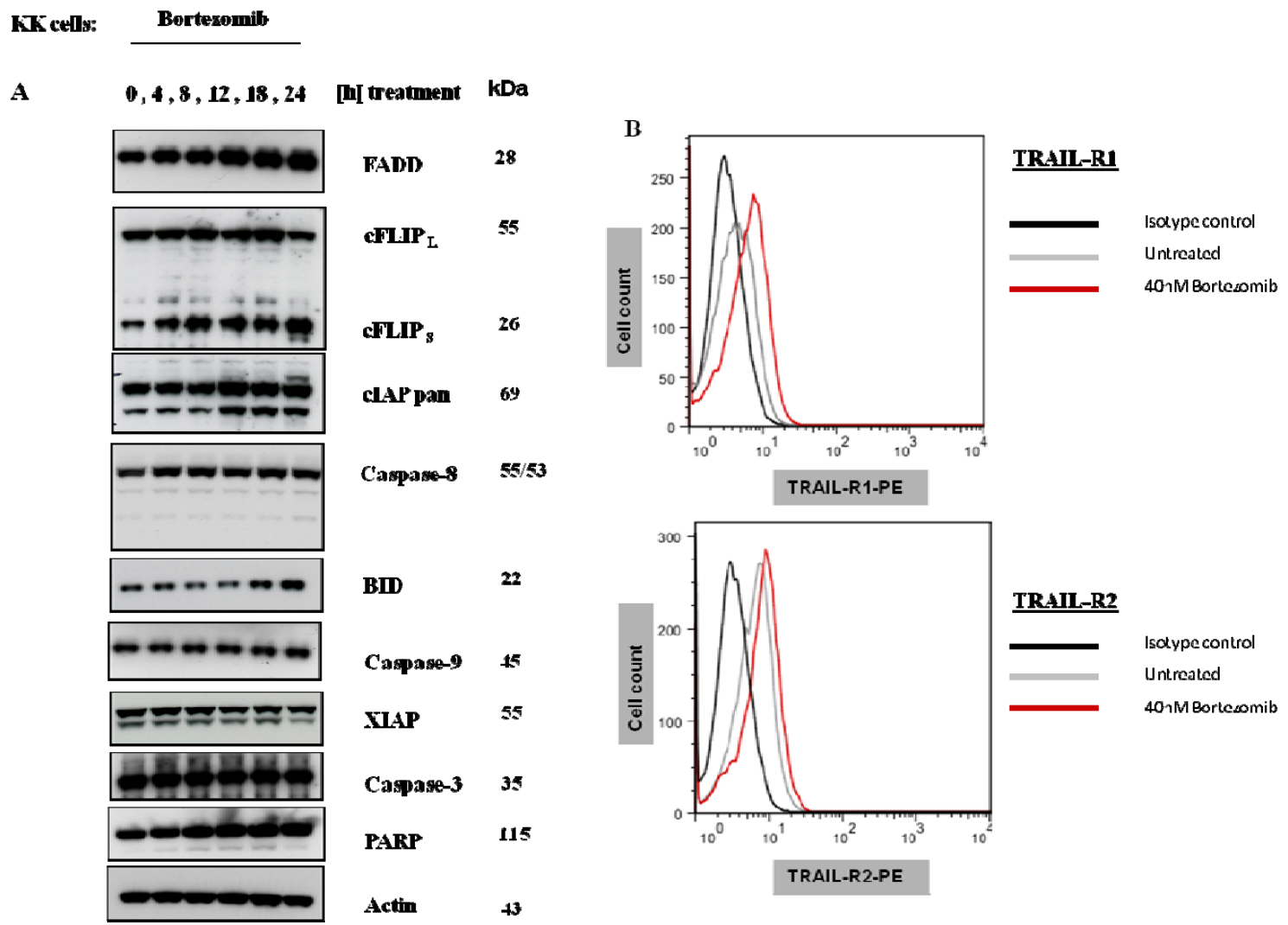

Figure 18.1: Bortezomib changes the expression pattern of different molecules along the TRAIL signalling pathway: A. $2 \times 10^{5} \mathrm{KK}$ cells were treated with $40 \mathrm{nM}$ Bortezomib for the indicated time. Cell lysates were prepared as described in chapter 3 and WB analysis was performed. B $1 \times 10^{5} \mathrm{KK}$ cells were treated with $40 \mathrm{nM}$ Bortezomib for $24 \mathrm{~h}$. Cells were subsequently stained for TRAIL-R1 and -2 and receptor expression levels were measured by Flow Cytometry as described in chapter 3 and compared to the Isotype control (mIgG1).

The WB analysis of PEA-1 and PEA-2 cells upon PIK75 or Smac83 treatment showed a very strong down-regulation of $\mathrm{cFLIP}_{\mathrm{L}}, \mathrm{cFLIP}_{\mathrm{S}}$ and cIAPs, respectively [Figure 18.2A,B].

Already after $4 \mathrm{~h}$ of PIK75 treatment in PEA-1 cells and $8 \mathrm{~h}$ in PEA-2 cells a potent downregulation of $\mathrm{cFLIP}_{\mathrm{L}}$ and $\mathrm{cFLIP}_{\mathrm{S}}$ could be detected as well as a strong downregulation of XIAP. cIAP-1/-2 showed a slight downregulation after $18-24 \mathrm{~h}$ but only in PEA-2 cells. Again, no caspase cleavage was detected upon inhibitor-only treatment. The Smac mimetics kinetic was performed with two different compounds-Smac83 and Smac59. Smac83 is an optimised version of Smac59, and the activity of both compounds needs to be compared. The comparative kinetic showed no differences between the two compounds so that in this context they can be used interchangeably. After treatment with Smac59 or Smac83 a rapid degradation of cIAP-1 and cIAP-2 could be observed. Surprisingly, eventhough cFLIPS 


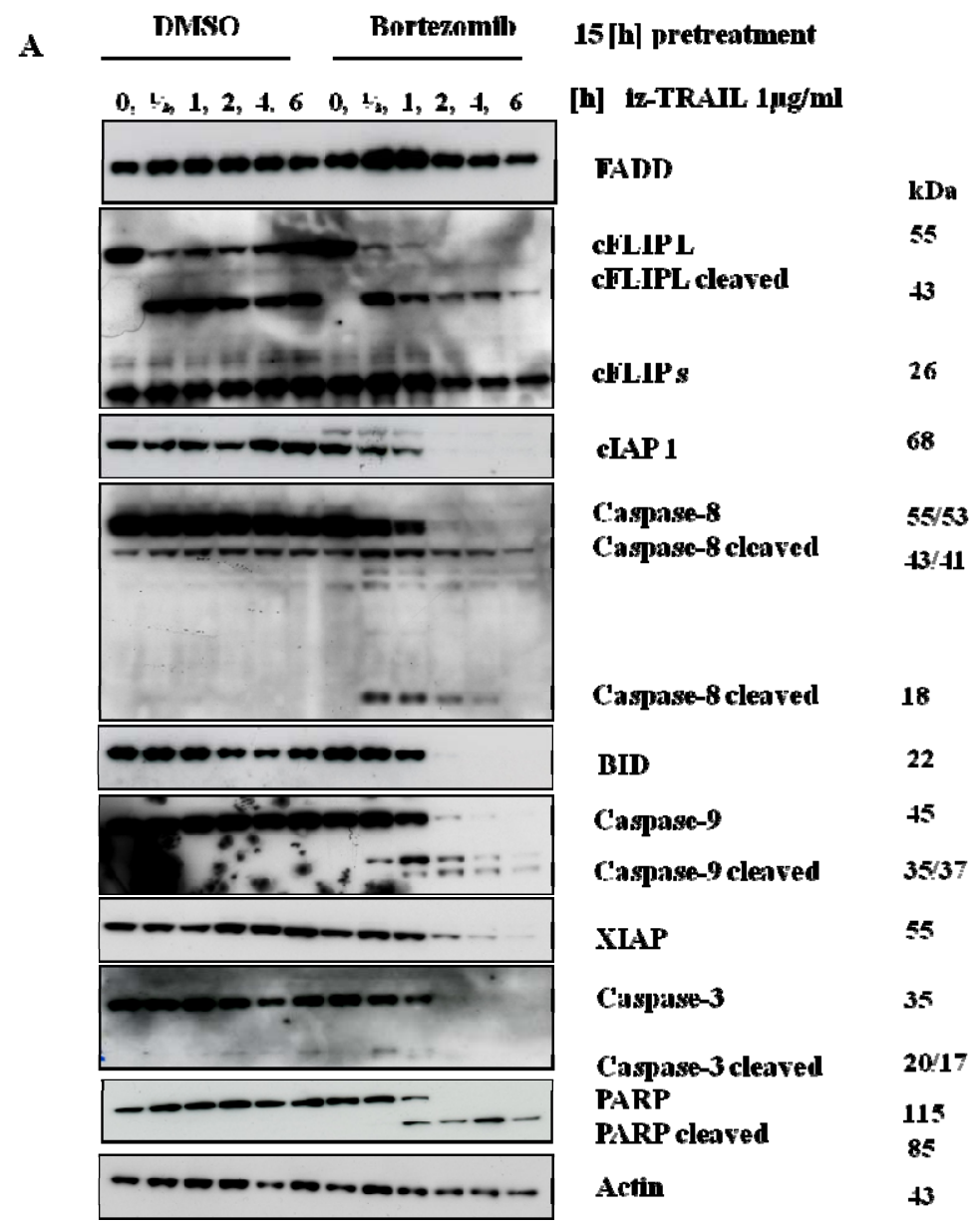

Figure 19.1: Processing of intracellular factors upon TRAIL treatment A. $2 \times 10^{5} \mathrm{KK}$ cells were pre-treated with 40nM Bortezomib for at least 15 hours. Cells were subsequently treated with TRAIL for the indicated time. Cell lysates were prepared a described in chapter III and WB analysis was performed.

PEA-1 cells were chosen for further analysis of molecules regulated by PIK75 or Smac83, as they are not as sensitive to TRAIL alone as their isogenic match PEA-2 and sensitisation effects are stronger.

Therefore, PEA-1 cells were pre-treated for 15 hours with either PIK75 or Smac83 or with the DMSO control [figure 19.2].

PEA-1 cells already show processing of molecules in the TRAIL cascade with TRAIL treatment alone. However, cells pre- treated with PIK75 or Smac83 exhibited increased cleavage of $\mathrm{cFLIP}_{\mathrm{L}}$ at the DISC. In order to further elucidate the downregulation of cIAPs that was observed in the PIK75-only kinetic, antibodies that are specific for either cIAP-1 or cIAP-2 were used in this WB analysis, instead of the pan-cIAP antibody. The WB shows that cIAP-2 is downregulated by PIK75 but cIAP-1 remains largely unaffected. Already after two 
hours of Smac83 and PIK75 treatment,c- 8 was completely cleaved (p18 fragment). In PEA-1 cells pre-treated with DMSO full cleavage of caspase- 8 could not be detected earlier than after eight hours. In addition, cleavage of Bid was enhanced in Smac83 or PIK75 pre-treated cells. Cleavage of caspase-9 occurs after 2 hours in all treatment groups. However, PARP cleavage as the ultimate sign of apoptosis is rather incomplete in DMSO control cells, whereas PARP can be completely cleaved in PIK75 and Smac83 pre-treated cells after eight hours. Furthermore, Smac83 on the one side leads to potent degradation of cIAP-1 and cIAP-2 allowing for cell death to occur, but on the other side up-regulating XIAP which acts anti-apoptotically. PIK75 treated cells show a very strong down-regulation of $\mathrm{cFLIP}_{\mathrm{L}}$ and cFLIP $_{\mathrm{S}}$, as compared to control cells and Smac83 treated cells. Upon TRAIL treatment $\mathrm{cFLIP}_{\mathrm{L}}$ and $\mathrm{cFLIP}$ are completely degraded.

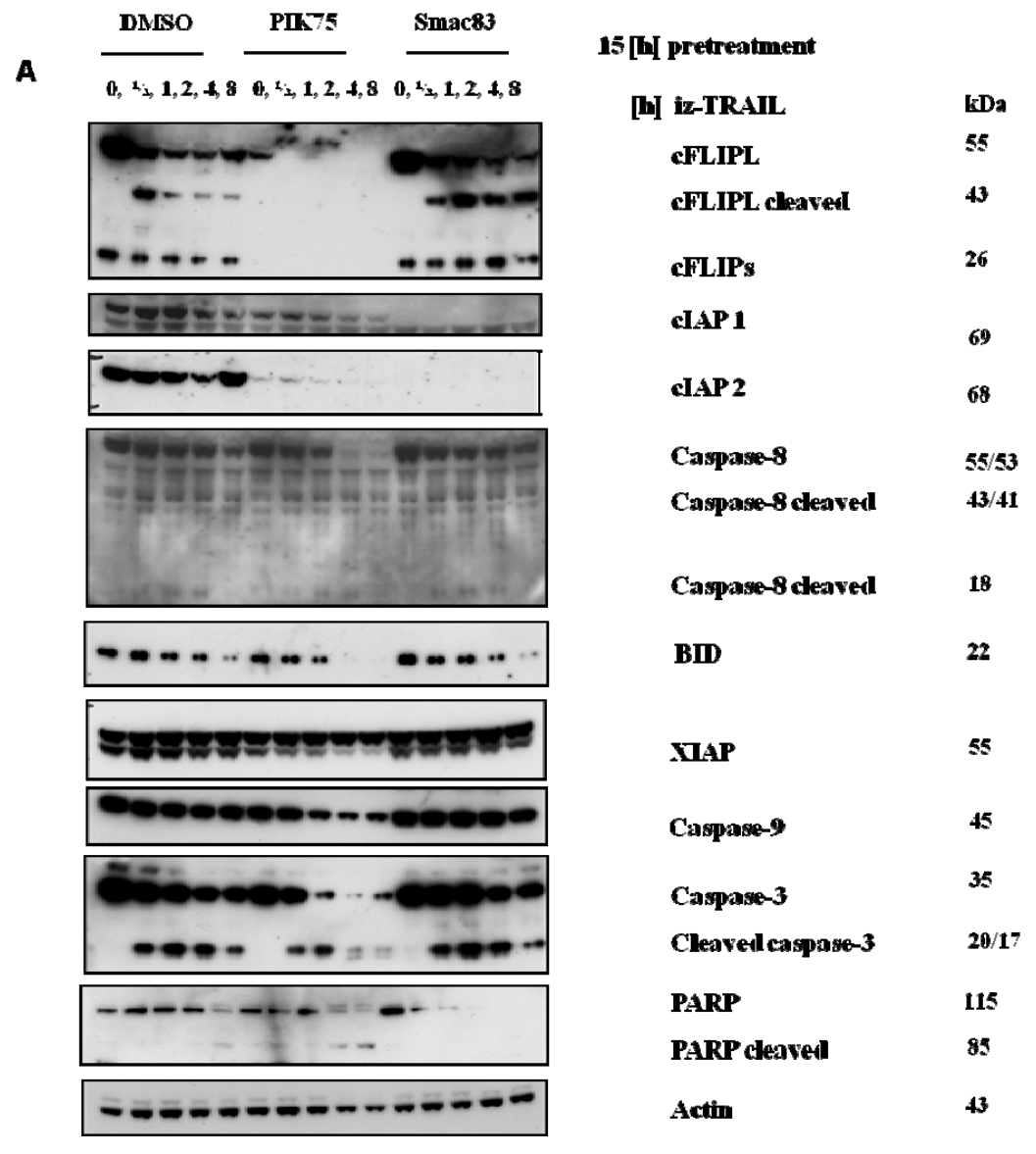

Figure 19.2: Processing of intracellular factors upon TRAIL treatment A. $2 \times 10^{5}$ PEA- 1 cells were pretreated with 100nM PIK75 or Smac83 for at least 15 hours. Cells were subsequently treated with TRAIL for the indicated time. Cell lysates were prepared a described in chapter III and WB analysis was performed. 
Since it has been shown that Bortezomib leads to an up-regulation of FADD and down-regulation of cFLIP (Ganten et al. 2004), the composition of the TRAIL DISC upon Bortezomib treatment should be investigated.

In order to be able to investigate the effects that Bortezomib might have on the DISC of KK cells a modified Tandem Affinity Purification TRAIL had to be produced (See chapter 3.2.5).

\subsection{Bortezomib treatment affects the composition of the TRAIL-DISC}

As previously published by our lab, Bortezomib treatment leads to an increase in DISC components expression and recruitment. The question arose, whether cFLIP, FADD and caspase- 8 where the only factors regulated or whether new factor could be identified at the DISC which might play a role in the sensitisation to TRAIL-induced apoptosis by Bortezomib. Having shown, that the newly purified moTAP-TRAIL specifically led to apoptosis induction in HCT-116 cells, which can be rescued by TRAIL-R2-Fc protein preincubation and furthermore can be used for immuno-precipitation it could now be employed for the analysis of the TRAIL-DISC with or without Bortezomib treatment in KK cells. The TRAIL DISC was precipitated with M2 beads, only. Figure 20A shows that both TRAIL-R1 and TRAIL-R2 are present in the immuno-precipitated samples. As already shown in figure 18.1.B, Bortezomib pre-treatment leads to an up-regulation of TRAIL-R1 and TRAIL-R2 in the immuno-precipitated cells, as well as in the TCL. Furthermore, there is a very potent upregulation of FADD and caspase-8 detectable, which is a lot stronger in the immune-precipitated samples than in the TCL, indicating a more efficient recruitment of Bortezomib treated cells.

The presence of cFLIP in the DISC is equal or even less in DMSO and Bortezomib pretreated cells, if the receptor cFLIP ratio is considered.

Furthermore, the recruitment of cIAPs into the DISC has just very recently been observed in our laboratory (unpublished data). Upon Bortezomib treatment cIAP levels are strongly diminished in the DISC, whereas their expression levels in the TCLs remains almost unchanged by Bortezomib. 


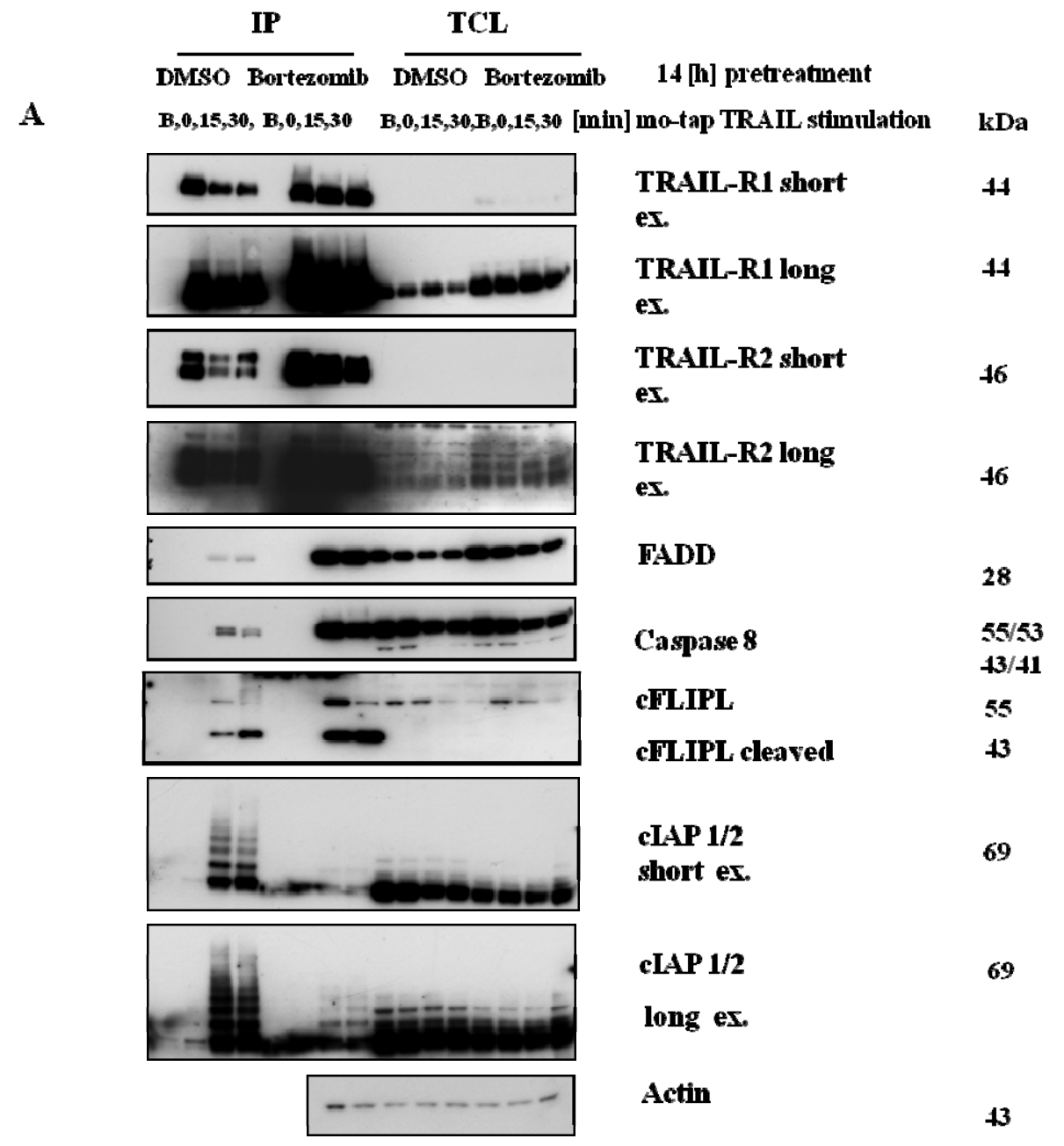

Figure 20: Bortezomib treatment affects the composition of the DISC: A. Cells that had been pre-treated with either 40nM Bortezomib or DMSO control were then stimulated with moTAP-TRAIL for 0, 15 or 30 min (0'15'30') or left unstimulated ( $\mathrm{B}=$ Beads only). Cell lysates were prepared and the TRAIL receptor complex was immuno-precipitated with M2 beads, $30 \mu 1$ of lysates were removed before immuno-precipitation to control for equal expression of protein levels (Total cell lysates=TCL)

\subsection{0. cIAPs are recruited into the DISC independently of FADD}

In order to investigate the level at which cIAPs are recruited to the DISC and thereby investigate at which step Bortezomib-treatment affects their presence in the DISC, DKO4 cells overexpressing dominant-negative (dn) mutant of FADD were used as a tool. Dn FADD is a truncated version of FADD comprising only the amino-terminal DD of FADD. This results in abrogation of the recruitment of caspase- 8 or cFLIP into the DISC. 
To further elucidate the mechanism of recruitment of cIAPs into the DISC, DKO4 control, and DKO4 dn FADD cells were compared in an immuno-precipitation. As shown in figure 21A, dnFADD DKO4 cells are rescued from apoptosis since they cannot assemble a DISC, which is one of the hallmarks of TRAIL-induced apoptosis (Kischkel et al. 2000; Sprick et al. 2000).
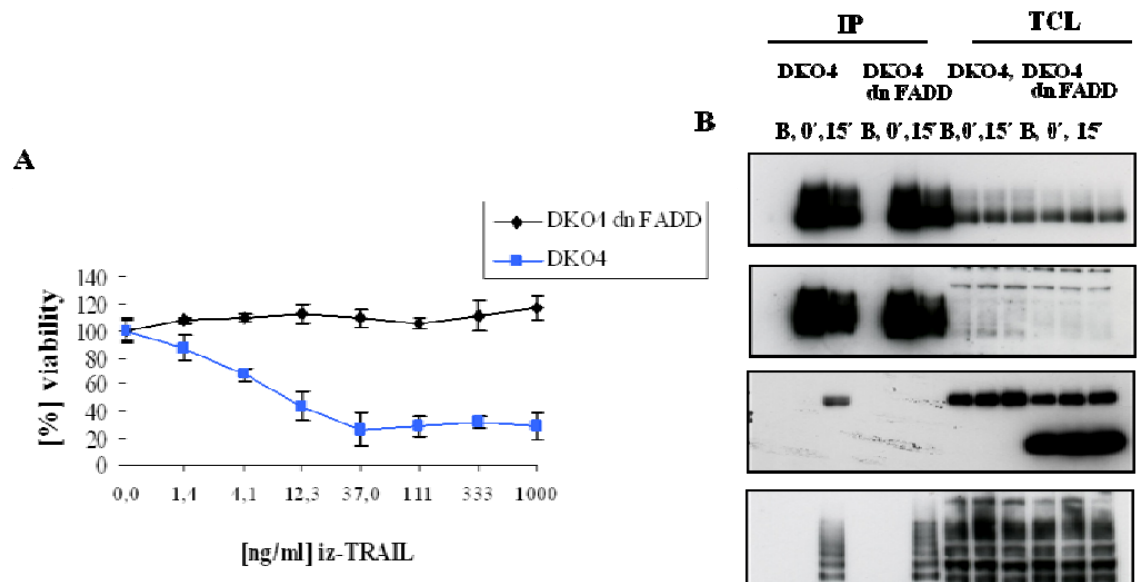

$\mathbf{B}$

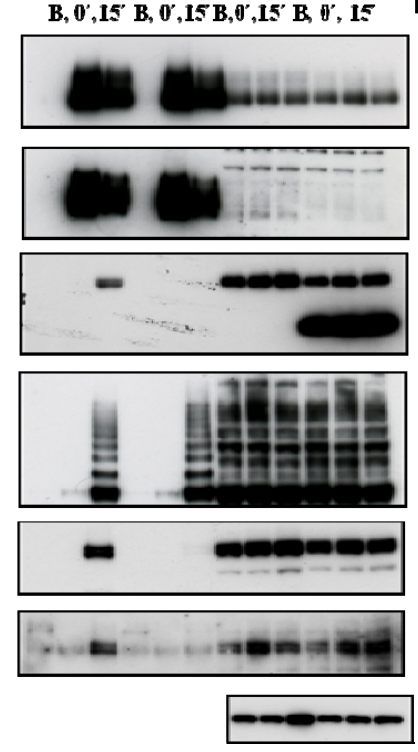

[min] mo-tap TRAII stimulation

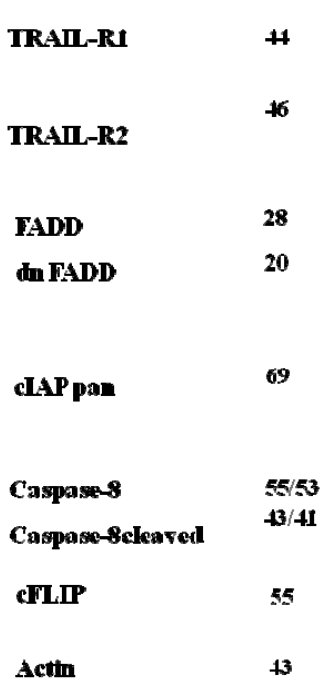

Figure 21: cIAPs are recruited to the DISC upstream of FADD A. DKO4 cells and DKO4 cells expressing dominant negative FADD were seeded in a 96-well plate and stimulated with increasing amounts of iz-TRAIL for $24 \mathrm{~h}$. Cell viability was measured by MTT assay. Results were generated from triplicate values.

DKO4 cells or DKO4 cells expressing dominant negative FADD were stimulated with $1 \mu \mathrm{g} / \mathrm{ml}$ moTAP-TRAIL for the indicated timepoints. Cell lysates were prepared and the TRAIL receptor complex was immunoprecipitated with M2 beads, $30 \mu 1$ of lysates were removed before immuno-precipitation to control for equal expression of protein levels (Total cell lysates=TCL).

As shown in Figure 21B, both TRAIL-R1 and TRAIL-R2 are present at similar levels in the immuno-precipitated samples of DKO4 cells and dnFADD DKO4 cells. In fact, caspase- 8 or cFLIP recruitment to the DISC is abrogated in dnFADD DKO4 cells, in contrast to normal FADD expressing DKO4 cells. Surprisingly, dnFADD was not detected in the DISC, whereas it was strongly detectable in the TCL. Importantly, the recruitment of cIAPs into the DISC is unchanged in dnFADD expressing DKO4 cells, so that it can be reasoned, that cIAPs must be recruited independent of FADD and caspase-8. 


\subsection{Isolation of ovarian cancer cells from ascitic fluid}

As it has now been shown that chemoresistant cell lines can be sensitised to TRAIL-induced apoptosis with Bortezomib, PIK75 and Smac83 the significance and importance of the tested treatment combinations should now be tested in primary ovarian cancer cells that are mostly chemoresistant. Primary ovarian cancer cells were to be treated after isolation from ascitic fluid. Here, all patients presented with relapsed ovarian cancer and had already received chemotherapy treatment.

Hence, the isolation and purification of ovarian cancer cells from ascitic fluid was established as a next step in this thesis.

EpCAM was shown to be highly expressed on over $90 \%$ of ovarian cancer cells (Diaz-Arias et al. 1993). EpCAM can also be expressed on normal epithelial cells, however, within the peritoneal cavity, EpCAM expression can be considered as tumour-specific as normal cells in the peritoneal compartment are of mesothelial origin and do not express EpCAM on their surface (Burges et al. 2007). As a first step of the isolation process, erythrocytes were depleted from the fluid via a ficoll gradient. If necessary the erythrocytes were subjected to lysis in a second step. Ficoll separation could possibly also result in the reduction of tumour cell yield. Nevertheless, we proceeded using Ficoll separation, as without Ficoll separation, the cellular debris and erythrocytes can block the magnetic beads and thereby decrease the yield and purity of the enrichment. Generally, volumes of one liter of ascitic fluid were received from the nearby hospital.

For the optimisation of the EpCAM isolation the EpCAM expressing cancer cell line IGROV-1 was used (R. Agarwal personal communication). IGROV-1 cells were incubated with Epithelial Enrich Dynabeads ${ }^{\circledR}$ according to the manufacturer's instructions (see chapter 3.2.4.). IGROV-1 cells with and without Dynabeads ${ }^{\circledR}$ were subjected to killing by iz-TRAIL in order to confirm, that Dynabeads ${ }^{\circledR}$ do not alter the outcome of the MTT-assay we used [Figure 22A]. Furthermore, it was shown, that proliferation is not influenced by the presence of Dynabeads ${ }^{\circledR}$ on the cells [Figure 22B].

Figure 22C,D show, that the morphology of IGROV-1 cells covered in EpCAM Dynabeads ${ }^{\circledR}$ is unchanged, the cells are spreading normally on tissue culture plastic and are establishing cell-to-cell contacts with neighbouring cells. 
Staining of EpCAM on IGROV-1 cells further confirms the finding, that the vast majority of IGROV-1 cells express the EpCAM antigen on their surface [Figure 22E], by which they can successfully be isolated by EpCAM Dynabeads $\AA$.

As there might be only few EpCAM positive cells in ascitic fluid, the assay that was going to be used for primary ovarian cancer cells not only needed to be a high troughput assay but additionally as precise as possible even at small numbers of cells present. For that reason the commonly used MTT-assay was compared with the cell titer glo ${ }^{\circledR}$-assay which measures the amount of ATP present which is proportional to viable cells.

Two setups were performed in parallel, seeding decreasing amounts of cells into a 96-well plate using the same cell batch for both assays. Each time, 50\% less cells were seeded, so that the cell viability should be decreased by $50 \%$. As shown in Figure 22F, G the signal in the cell titer glo ${ }$-assays nicely decreased by $50 \%$ down to 750 cells/ well $(\sim 7500 / \mathrm{ml})$ whereas the MTT assay is only accurate down to $\sim 5000$ cells/well.

Taking these results together, we applied the cell titer glo®-assay for cells isolated from ascitic fluid.

i
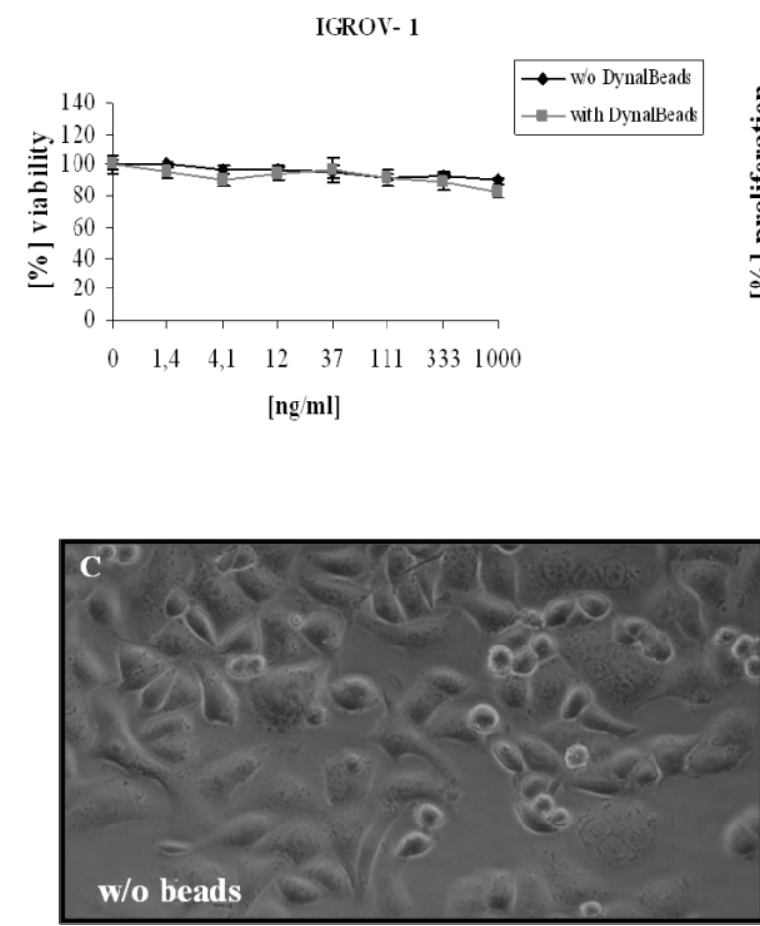

B
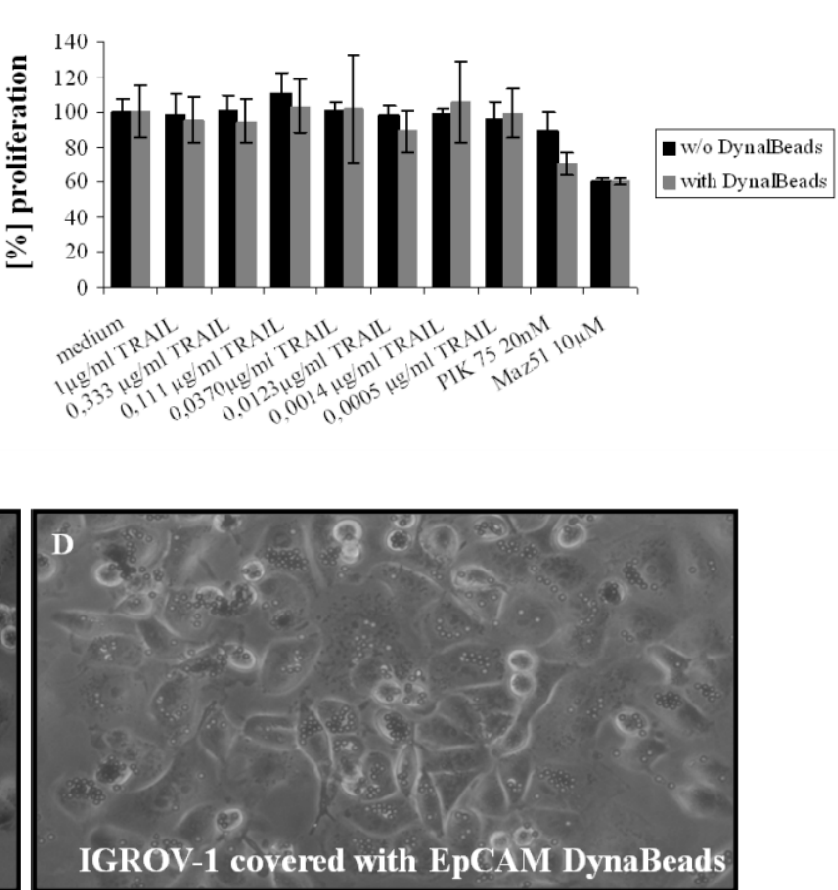
$\mathbf{E}$

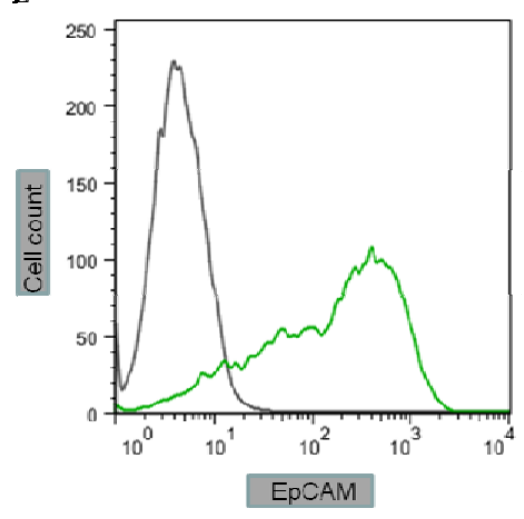

$\mathbf{F}$ Cell Titer Glow

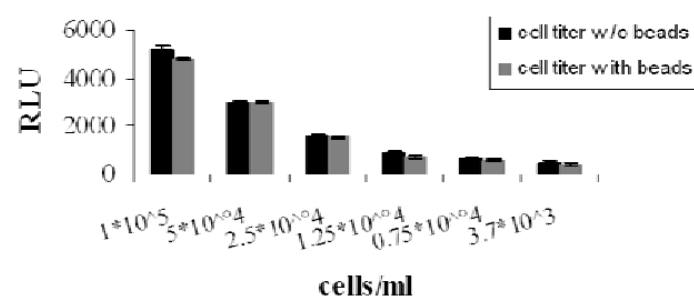

G
- MTT w/obeads a MT with beads

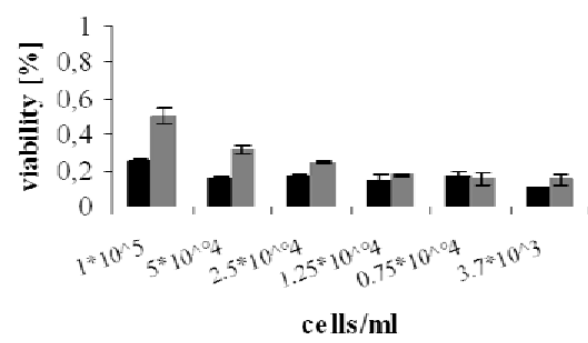

Figure 22: Use of ovarian cancer cell line IGROV-1 to establish of isolation of ovarian cancer cells from ascitic fluid with EpCAM Dynabeads. A. IGROV-1 cells were either incubated with Epithelial Enrich Dynabeads ${ }^{\circledR}($ EpCAM) or left untouched. They were subsequently stimulated with increasing amounts of izTRAIL for $24 \mathrm{~h}$. Cell viability was measured by MTT assay. B. IGROV-1 cells with or w/o EpCAM Dynabeads were treated with bromodeoxyuridine (BrdU) for 72h. C. IGROV-1 cells with or w/o EpCAM Dynabeads were seeded on normal cell culture plastic to observe, whether they have a changed morphology after isolation with EpCAM Dynabeads. D. IGROV-1 cells were stained with an IgG1 EpCAM-FITC coupled antibody for Flow Cytometry. One sample was stained with mIgG1 as a control (Isotype control) E. To compare cell titer glo ${ }^{\circledR}$ and MTT viability assays decreasing amounts of cells were seeded into a 96 well plate using the same reagent reservoir for both assays. Each time, 50\% less cells were seeded, so that the relative luminescent untis (RLU) should be decreased by $50 \%$. Cells were incubated for 24 hours before cell viability was determined. 


\subsection{Primary ovarian cancer cells can be sensitised to TRAIL-induced apoptosis by Bortezomib}

Having shown in three different ovarian cancer cell lines that they can be potently sensitised to TRAIL-induced apoptosis with Bortezomib, we applied Bortezomib as a treatment of first choice to primary ovarian cancer cells isolated with EpCAM Dynabeads ${ }^{\circledR}$ from ascitic fluid, as well as to the control group of EpCAM negative cells, that were expected to be mainly normal cells.

As shown in figure 23A-C EpCAM positive ovarian cancer cells can specifically be sensitised to TRAIL-induced apoptosis with Bortezomib in three different patients with stage III ovarian cancer. TRAIL alone killed cancer cells to about $15 \%$, in all three patients. Bortezomib induced slight killing of about $20 \%$. When applied in combination with TRAIL a potent sensitising effect ranging from $40-70 \%$ loss of viability in EpCAM positive ovarian cancer cells could be detected. The EpCAM negative population which should comprise normal cells, as well as EpCAM negative cancer cells (Curley et al. 2009) also showed a fundamental sensitisation effect upon TRAIL and Bortezomib co-treatment, even though TRAIL treatment usually does not harm normal cells (Walczak et al. 1999). The sensitisation in EpCAM negative cells, however, is not as effective as in EpCAM positive cancer cells which led to the assumption that the EpCAM negative population must be a mixture of normal cells and EpCAM negative ovarian cancer cells that can not be isolated via the applied EpCAM Dynabeads ${ }^{\circledR}$ method indicating the necessity for an improved protocol for the isolation of ovarian cancer cells from ascitic fluid. 

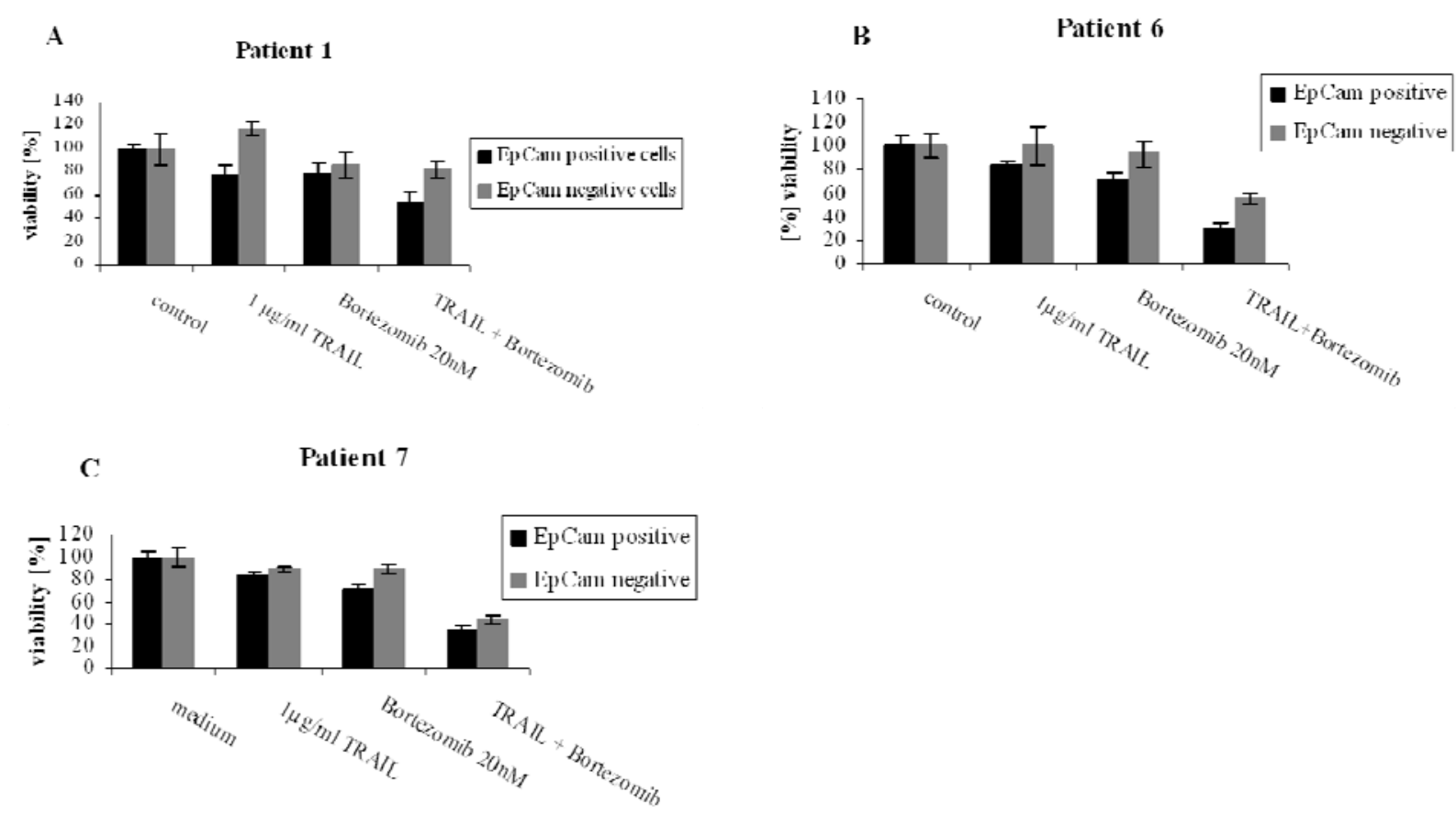

Figure 23: Bortezomib specifically sensitises primary ovarian cancer cells to TRAIL-induced apoptosis. AC. $1 \times 10^{4}$ EpCAM positiv or EpCAM negative cells isolated from ascitic fluid of patients with FIGO stage III ovarian carcinoma were incubated with $20 \mathrm{nM}$ Bortezomib and $1 \mu \mathrm{g} / \mathrm{ml}$ iz-TRAIL for $24 \mathrm{~h}$. Cell viability was measured by cell titer glo ${ }^{\circledR}$ assay. deviation. Values are mean $+/-$ standard deviation (SD) of one experiment which was performed in duplicates.

\subsection{Cisplatin specifically sensitises primary ovarian cancer cells to TRAIL-induced apoptosis}

Cisplatin is a known sensitiser for cancer cells to TRAIL-induced apoptosis, even in cisplatin resistant cancer cells. As shown in Figure 24A,B primary ovarian cancer cells that are resistant to single agent treatment with cisplatin can efficiently be sensitised to TRAIL by Cisplatin treatment.

The difficulty that has already been discussed regarding Figure 16 appears to be an issue once again as EpCAM negative cells are also susceptible to TRAIL treatment, even though far less than EpCAM positive cancer cells. 
A

patient 5

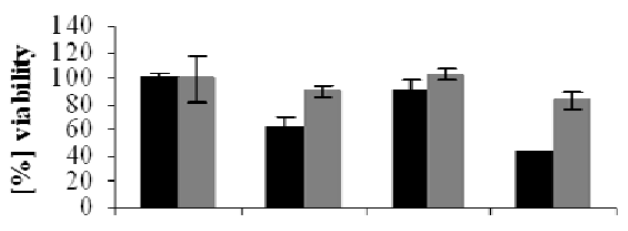

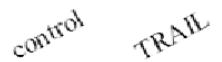

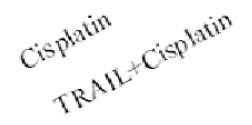

B

Patient 7

EpCAM positive

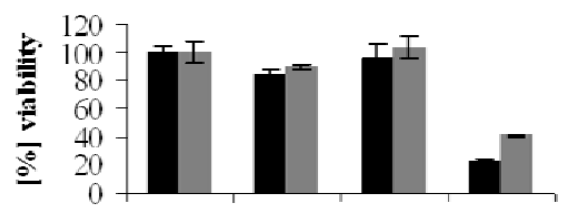

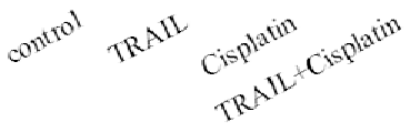

Figure 24: Cisplatin specifically sensitises primary ovarian cancer cells to TRAIL-induced apoptosis. A+B. $1 \times 10^{4}$ EpCAM positive or EpCAM negative cells isolated from ascitic fluid of patients with stage three ovarian carcinoma were incubated with $1 \mu \mathrm{g} / \mathrm{ml}$ Cisplatin and $1 \mu \mathrm{g} / \mathrm{ml}$ iz-TRAIL for $24 \mathrm{~h}$. Cell viability was measured by cell titer glo ${ }^{\circledR}$-assay. Experiment was performed in triplicates. Error bars represent SD.

\subsection{Optimisation of the isolation protocol for ovarian cancer cells from ascitic fluid}

Taken together, the results obtained from primary ovarian cancer cells isolated from ascitic fluid it can be stated, that EpCAM positive ovarian cancer cells can efficiently be sensitised to TRAIL-induced apoptosis via Bortezomib and Cisplatin treatment. However, so far there is no clear defined population containing only normal cells that can be used for toxicity studies of the different inhibitors and TRAIL. Hence, the protocol for the isolation needed further improvement and was optimised as shown in figure 25.1A.

After depletion of erythrocytes from the total cells population, this population was subjected to staining by Flow Cytometry, staining for CD45 (pan-leucocyte marker), CD14 (monocyte marker) and EpCAM. Figure 25.2A shows a representative example staining of a total cell population comprising a very heterogeneous population of big cells and small cells that mainly stain positive for CD45 $(\sim 50 \%)$, but also contain large amounts of CD45, CD14 double positive cells ( 28\%) labelling them as monocytes. Besides the CD45 positive population, there is a smaller but clearly defined EpCAM positive population detectable in the total cell population of approximately $18 \%$. 
Prior to the EpCAM isolation, the new protocol is expanded by a CD45 Dynabeads ${ }^{\circledR}$ isolation that holds at least two advantages. Firstly, the depletion of CD45 positive cells decreases the whole cell population by $\sim 50-80 \%$ (values vary from patient to patient), diminishing unspecific binding of CD45 positive cells to the EpCAM beads and secondly and most importantly the creation of a pure normal cell population containing different subpopulations of leucocytes.

As shown in Figure 25.2C in the FSC and SSC the CD45 positive population is a much more defined population of cells with the same size and granularity. CD45 positive cells stain positive for CD14 but not for CD45 as the antigen binding sites are saturated by CD45 Dynabeads ${ }^{\circledR}$.

Hence, Figure 25.2B shows a cell population that is largely depleted of CD45 positive cells yielding a very much enriched EpCAM positive population of ovarian cancer cells that can now be isolated with EpCAM Dynabeads ${ }^{\circledR}$ in order to even increase its purity.

The population that is neither stained positive for CD45 nor for EpCAM is subjected to fluctuations and again varies a lot among patients.

All in all, this optimised protocol accomplishes the purification of two defined populations of cancer and non-cancer cells that can now be subjected to TRAIL treatment.

$\mathbf{A}$

Optimised Isolation protocol:

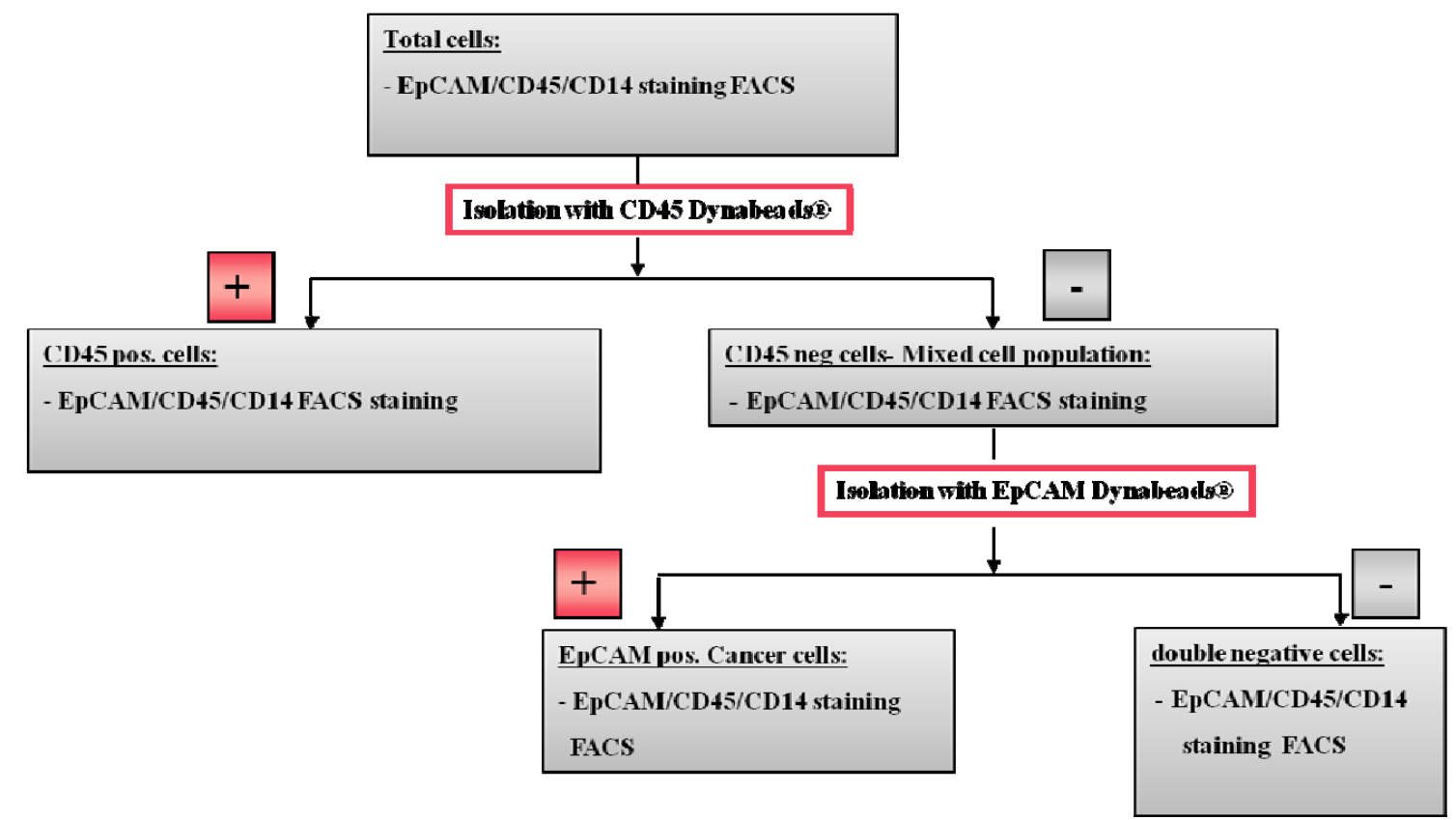

Figure 25.1: Diagram of new isolation protocol for the purification of ovarian cancer cells from ascitic fluid A. Cells are subjected to a first isolation step with CD45 Dynabeads ${ }^{\circledR}$ and a second step with EpCAM Dynabeads ${ }^{\circledR}$. 


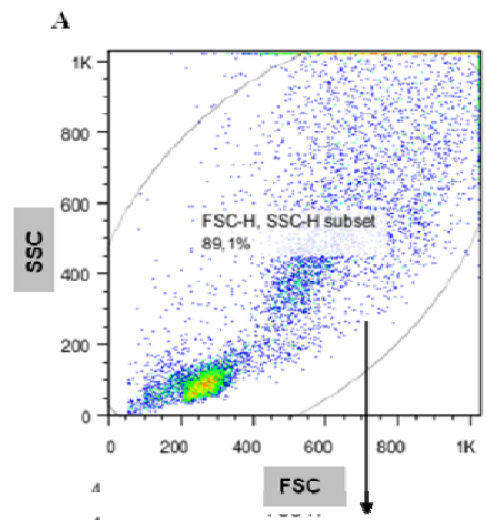

Total cells
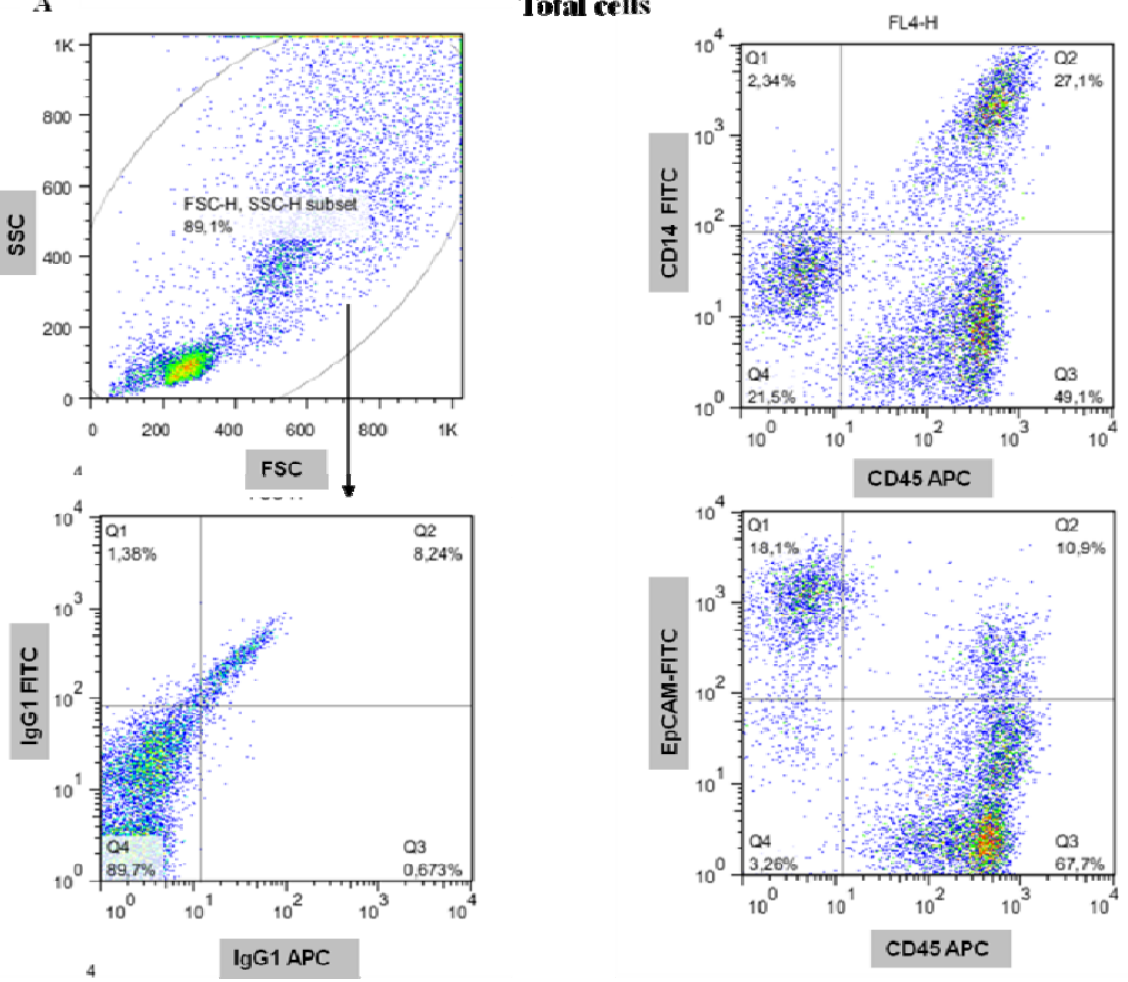

B

CD45 positive cells - Isolated with CD45 DymaBeads (i)
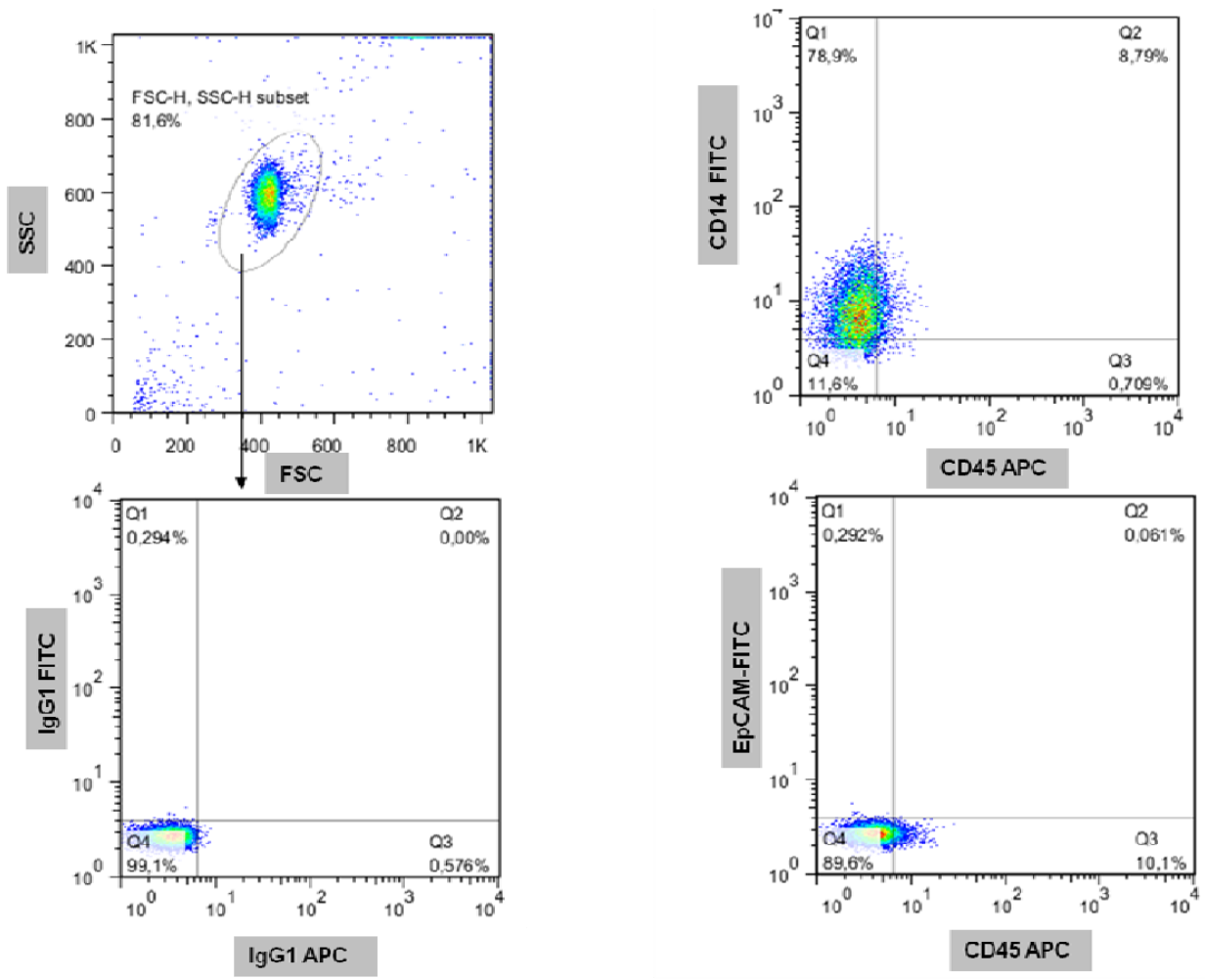
C

CD+5 negative cells
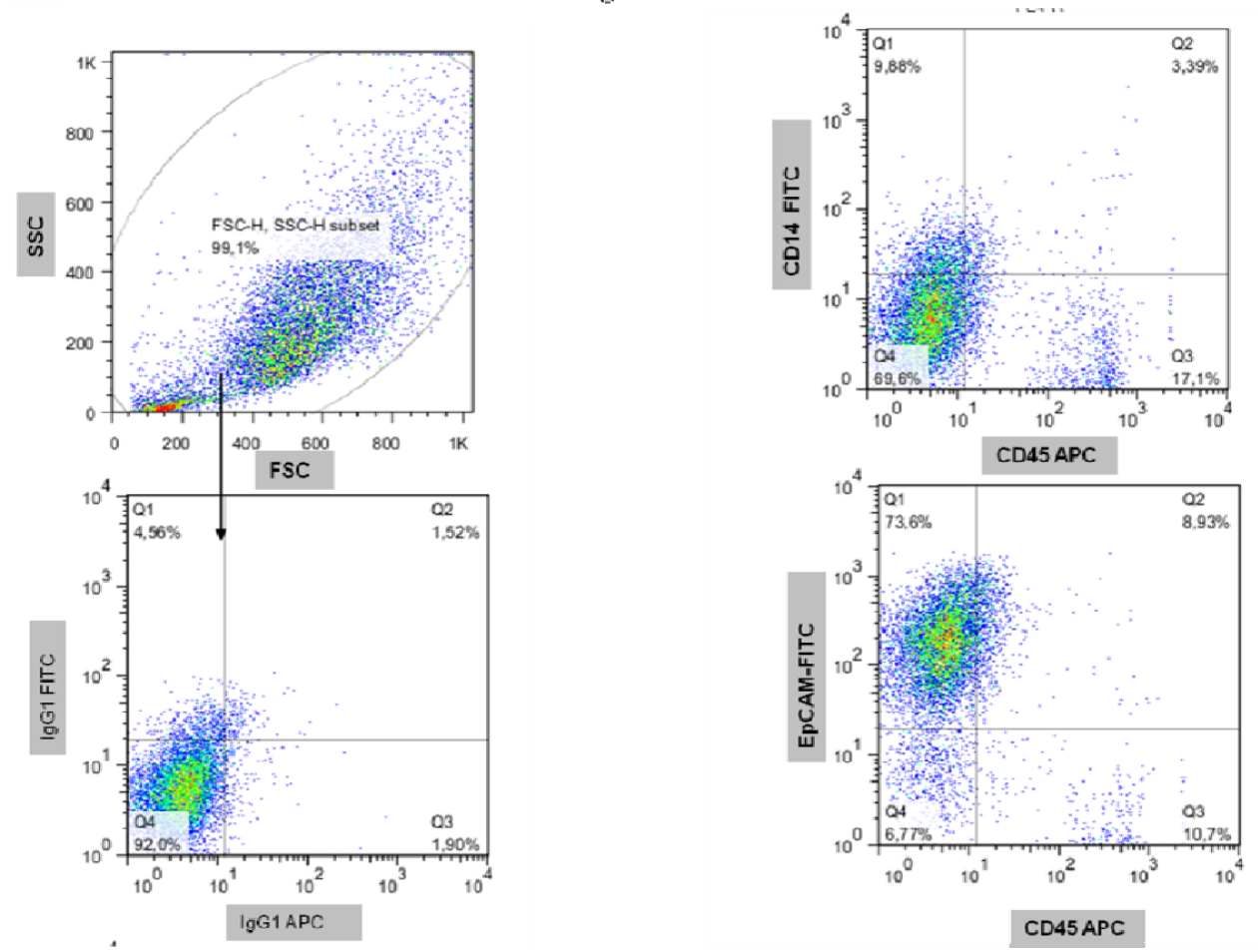

Figure 25.2: Optimisation of the isolation protocol for the purification of ovarian cancer cells from ascitic fluid A-C. $2 \times 104$ cells were incubated with Fc-block for 5 min and subsequently stained for Flow Cytometry with indicated antibodies. One sample was stained with mIgG1 as a control.

\subsection{EPCAM positive cancer cells but not CD45 positive leucocytes can be sensitised to TRAIL-induced apoptosis via small molecule inhibitors}

Applying the optimised protocol for isolating cancer cells from ascitic fluid and subjecting the different cell population to co-treatment of TRAIL plus inhibitors it can be stated that the TRAIL effect is cancer cells specific and is not toxic to CD45 positive non-cancer cells. As shown in figure 26A EpCAM positive ovarian cancer cells can be sensitised not only with Bortezomib, but also with PIK75 to TRAIL-mediated apoptosis. Again Bortezomib already slightly kills on its own, whereas PIK75 causes even less killing when applied as a single agent. These particular ovarian cancer cells exhibit absolute resistance to TRAIL alone, however, they can be effectively sensitised to TRAIL by co-treatment with the respective inhibitors. In contrast, the CD45 positive population is not affected by TRAIL and PIK75 plus TRAIL and only shows slight toxic effects upon Bortezomib stimulation. The double negative 
population, that is now depleted of most of its CD45 positive cells and of all its EpCAM cells, shows a similar behaviour as the EpCAM positive cancer cells, suggesting that it mainly contains EpCAM negative cancer cells.

\section{Patient 8:}

A

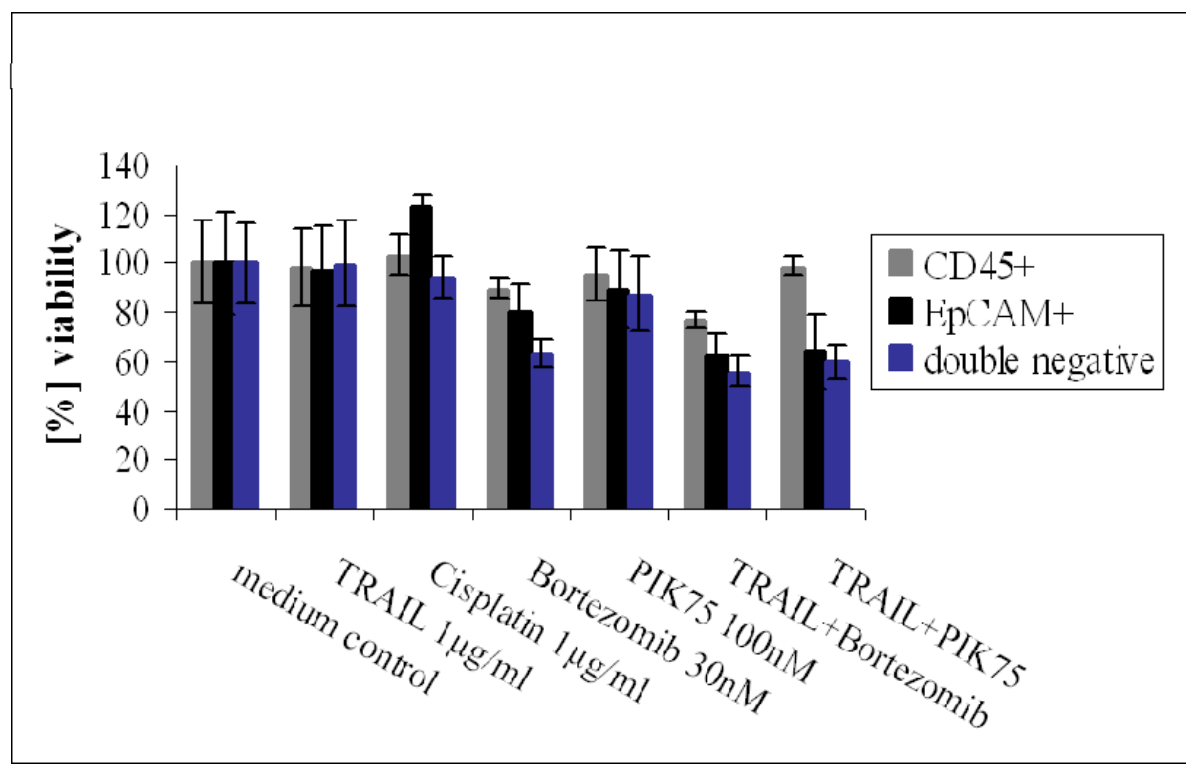

Figure 26: Primary ovarian cancer cells can be sensitised with to TRAIL-induced apoptosis with different sensitising agents. A-C. $1 \times 10^{4}$ EpCAM positive, CD45 pos. or double negative cells isolated from ascitic fluid of patients with stage three ovarian carcinoma were co-incubated with 30nM Bortezomib, 100nM PIK75 and $1 \mu \mathrm{g} / \mathrm{ml}$ iz-TRAIL for $24 \mathrm{~h}$. One sample was incubated with $1 \mu \mathrm{g} / \mathrm{ml}$ cisplatin for 72 hours. Cell viability was measured by cell titer glo ${ }^{\circledR}$ assay. Experiment was performed in triplicates. Error bars represent SD. 


\section{Discussion}




\section{Discussion}

Among the members of the TNF signalling family the TRAIL/TRAIL-R system has received special attention due to its tumour specific killing activity. However, the majority of primary tumours and 50\% of cancer cell lines have acquired resistance to TRAIL-mediated apoptosis. Yet, most of them can be sensitised with chemotherapy, radiotherapy or small molecule inhibitors that allow for effective use of TRAIL in first clinical trials in cancer treatment.

Currently, the gold standard for treatment of ovarian cancer is debulking surgery followed by primary chemotherapy with a platinum-containing regimen irrespective of the histopathological subtype of the ovarian carcinoma. Common side effects, such as neurotoxicity, alopecia and fatigue have a severe impact on the quality of life of these patients. Despite good initial response to systemic therapy the long-term survival remains poor with a high risk of recurrence. In order to reduce the toxicity of first-line chemotherapy and confine the recurrence of ovarian cancer, patients have been involved in worldwide randomised clinical trials. However, it is important to focus on the development of treatment options for each distinct histopathological subtype and especially find novel drugs for tumours that have acquired resistance to conventional chemotherapy.

\subsection{TRAIL sensitivity does not correlate with histologicals of ovarian cancer}

For the characterisation of the responsiveness of different ovarian cancer cell lines to TRAIL-induced apoptosis a highly active form of TRAIL, tagged with an iz-motive was used that leads to trimerisation of the ligand which increases receptor oligomerisation upon binding (Walczak and Haas 2008). No correlation could be found between histopathological subtype of a distinct ovarian cancer cell lines and its sensitivity to TRAIL-induced apoptosis. For instance, among the serous ovarian cancer cell lines, PEO-1 and PEO-4 were extremely TRAIL sensitive, whereas IOSE-80 and PEA-2 cells were partially TRAIL sensitive and PEA-1 cells exhibited complete TRAIL resistance [figure 12]. This might be because classification of histological subtypes relies only on microscopic characteristics of the respective cancer and TRAIL-resistance is expected to go along with specific mutation patterns of cancer. Although histological subtypes of ovarian cancer have some oncogenic 
mutations in common in the respective classes causing their phenotype, the same phenotype could also be caused by another set of mutations.

However, as the PI3K/Akt pro-survival-pathway has been reported to cause TRAIL resistance (Xu et al. 2010) and endometrioid ovarian cancers often harbour activating mutations of the PI3K/Akt pathway and/or inactivating mutations or deletions of PTEN that counteract activation of the PI3K pathway a correlation between specific mutations and TRAIL resistance in a particular subtype of ovarian cancer cannot be excluded. As endometrioid cancers are rare and therefore only one endometrioid ovarian cancer cell line was available (TOV-112D) it can not be stated whether TRAIL resistance is common in this specific subtype or not.

Clear cell ovarian cancers are a relatively aggressive subtype of ovarian cancer and account for $10 \%$ of ovarian cancers (Gilks 2010). The molecular origins of clear cell ovarian cancer remain undefined and as they show relatively low-mitotic rates (Kobel et al. 2008), they mostly do not respond to standard chemotherapy regimes that mainly target dividing cells. These findings highlight the acute need for novel therapeutic options, in particular in clear cell ovarian cancer subtypes.

\subsection{Potential sensitising agents exert variable toxicities in ovarian cancer cells lines}

While determining sub-toxic doses of the different inhibitors employed in sensitising TRAIL resistant ovarian cancer cell lines it was found that TOV-112D and PEA-2 cells were substantially more susceptible to loss of viability by proteasome inhibition (Bortezomib, PS341) and PI3K-inhibition (PIK75) alone, than PEA-1 and KK cells [figure 13]. Application of the smac-mimetic Smac59 led to a slight loss of viability in all three cell lines, which can be explained by their lack of autocrine tumour necrosis factor- $\alpha$ (TNF- $\alpha$ ) production, which has been shown to be responsible for smac-mimetic-induced death (Probst et al. 2010).

The concept of cytotoxic drugs is the integration of these drugs into the metabolism of cells, causing higher toxicity to those cells that have a higher growth rate. Consequently, one would assume, that TOV-112D cells with an average doubling time of 20 hours are more susceptible to cytotoxic drugs than PEA-1 or PEA-2 cells with a doubling time of 37 and 66 hours, respectively (Langdon et al. 1988). This assumption holds true for TOV-112D, PEA-1 and KK cells, however, PEA-2 cells that have the longest doubling time of all four cell lines show 
similar toxicity effects as TOV-112D cells. As this experiment was analysed by MTT assay, the differences seen in the viability might also be due to growth inhibition. An apoptosis specific assay could distinguish between growth inhibition and apoptosis induction.

\subsection{Implications of Bortezomib-induced sensitisation to TRAIL}

After determination of sub-toxic concentrations of possible sensitising agents in the particular cell lines, the appropriate concentrations in combination with increasing concentrations of iz-TRAIL were administered. Apoptosis was determined by measurement of sub-diploid DNA content and furthermore by inhibition of caspases by broad-spectrum caspase inhibitor zVAD-fmk and Q-VD-Oph. This specifically confirmed the induction of apoptosis in the two TRAIL-resistant cell lines PEA-1 and KK as well in the partially sensitive cell line PEA-2 [figure 14-16] showing that Bortezomib treatment is not only potent in further sensitising TRAIL sensitive cells but also has the ability to sensitise cells that are completely resistant to TRAIL-induced apoptosis. Most importantly, the sensitisation with Bortezomib to TRAIL did not only occur in cisplatin sensitive PEA-1 cells, but also in cisplatin resistant PEA-2 and KK cells. As a matter of fact, PEA-2 cells could even be more effectively killed with Bortezomib and TRAIL co-treatment than PEA-1 cells, showing that a TRAIL-based therapeutic regime is even particularly effective in cells that are otherwise chemoresistant.

This is an important finding, as patients with ovarian cancer even after complete clinical response, often relapse with platinum resistant disease. Acquired chemotherapy resistance is one of the greatest clinical challenges in the treatment of women with ovarian carcinoma. Hence, it is of the utmost importance to specifically develope novel drug combinations for these patients. Different mutation patterns have been described that can convey platinum resistance in patients with ovarian cancer. Germline mutations of BRCA1/2 genes confer inherited susceptibility to ovarian and breast carcinomas. However, in vitro studies indicate that BRCA1/2 loss increases sensitivity to agents that cause double strand DNA breaks and/or interstrand DNA cross-links including platinum agents (Husain et al. 1998; Bhattacharyya et al. 2000; Quinn et al. 2003). This would explain the improved survival for women with hereditary ovarian cancer in comparison to patients with sporadic ovarian carcinoma. Regarding sporadic ovarian cancer it was reported from evaluation of a large number of sporadic and advanced stage carcinomas that BRCA1 loss was strongly protective for the overall survival of patients (Thrall et al. 2006). 
Recently, it was shown that recurrent ovarian carcinomas commonly have increased BRCA1 and /or BRCA2 protein expression post chemotherapy exposure (Swisher et al. 2009) which could mediate resistance to platinum based therapy regimes. The mechanisms responsible for these expression differences remain unknown. Furthermore, p53 mutations, mostly missense mutations, are the most frequent mutations occurring in ovarian cancers. Tumours that possess mutations in intracellular apoptosis-sensing molecules like p53 and BRCA1/2 are not capable of inducing apoptosis on their own and are therefore likely to be resistant to chemotherapy. TRAIL-induced apoptosis, however, is achieved by directly triggering apoptosis from the outside of tumour cells, thereby circumventing chemotherapeutic resistance.

PEA-1, PEA-2, KK and TOV-112D cells were selected for further experiments as they each represent one distinct histopathological subtype of epithelial ovarian cancer and reside in different stages of TRAIL resistance. Furthermore, PEA-1 and PEA-2 cells are an isogenic matched pair, of which one has acquired cisplatin resistance, so they were an interesting experimental system to possibly detect differences in their ability to react to TRAIL and small molecule inhibitors.

Bortezomib has been approved in 2006 for the treatment of mantle cell lymphoma in patients that had received at least one prior therapy and in 2008 for the initial treatment of patients with multiple myeloma.

Co-treatment of Bortezomib and carboplatin has been applied in a Phase Ib clinical trial to treat patients with recurrent platinum and taxane resistant ovarian carcinomas (Ramirez, Landen et al. 2008). In this study, however, several patients presented with dose limiting toxicities (grade 3 ) that were attributable to relatively high concentrations of Bortezomib. The advantage of using a treatment regime comprising TRAIL and Bortezomib is that each drug itself will be applied in subtoxic concentrations but then synergises in patients to potently and specifically kill tumour cells, while normal cells are not effected.

KK cells were chosen, because clear cell ovarian cancer displays a small but very aggressive population of epithelial ovarian cancers for which treatment options are rare as they don't respond to chemotherapy regimes (Sugiyama et al. 2000; Itamochi et al. 2002; Crotzer et al. 2007). After confirming, that Bortezomib-mediated sensitisation to TRAIL specifically induces apoptosis [figure 14-16] in cisplatin resistant ovarian carcinoma cell lines, the regulation of pro-and anti-apoptotic molecules in KK cells was monitored [figure 18.1]. First 
of all, an up-regulation of FADD and BID could be detected in the total cell lysates. FADD has been shown to be more effectively recruited to the DISC upon Bortezomib stimulation in Hep3B cells without detectable regulation of FADD in the TCL (Ganten et al. 2005). However, the upregulation of FADD in the total cell lysates of KK cells is present but only minor when compared to the enhanced recruitment of FADD into the DISC upon Bortezomib pre-treatment.

The upregulation of BID upon Bortezomib treatment leads to enhanced availability of BID for cleavage. tBID can then activate Bax and Bak to induce MOMP. (Ganten et al. 2005). In fact, upon stimulation with TRAIL [figure 19.1], cleavage of BID occurred in Bortezomib pretreated cells but not in TRAIL-only treated cell. Increased Bid cleavage resulted in stronger activation of the mitochondrial pathway as supported by strong caspase- 9 and caspase- 3 cleavage after 30 minutes as well as XIAP cleavage after 2 hours TRAIL stimulation. Enhanced recruitment of FADD to the DISC leads to subsequent strong caspase- 8 recruitment to the DISC. Caspase-8 expression levels are unchanged in the TCL upon TRAIL stimulation. Strong caspase- 8 cleavage occurs at a very early time when cells are pre-treated with Bortezomib, which could be explained by the very much enhanced TRAIL-DISC formation upon Bortezomib treatment.

cFlip has been mostly shown to act as an inhibitor of TRAIL-induced apoptosis (Leverkus et al. 2000). However, it can also act pro-apoptotic. On the one side it was shown, that cFlip competes with caspase- 8 for binding at the DED of FADD thereby negatively influencing apoptosis induction (Irmler et al. 1997), whereas on the other side it was stated that cFlip forms active dimers with caspase- 8 at the DISC, allowing for release of active caspase- 8 and subsequent cleavage of apoptotic substrates (Micheau et al. 2002). The role of cFLIP at the DISC furthermore depends on its expression levels. Overexpression of $\mathrm{cFlip}_{\mathrm{L}}$ or cFlip resulted in inhibition of DISC formation and hence inhibited caspase- 8 processing (Scaffidi et al. 1999; Krueger et al. 2001). However, moderate expression levels lead to the opposite effect as they showed to enhance apoptosis induction through its protease like domain (Chang et al. 2002). In our setting, cFlips is strongly upregulated upon Bortezomib treatment however $\mathrm{cFlip}_{\mathrm{L}}$ is slightly downregulated after $24 \mathrm{~h}$. Despite the increase of cFlip $\mathrm{S}_{\mathrm{S}}, \mathrm{cFlip}$ L is rapidly and completely cleaved upon TRAIL stimulation which does not interfere with strong caspase- 8 cleavage after $30 \mathrm{~min}$. DMSO pretreated KK cells do not display complete cFlip $\mathrm{L}_{\mathrm{L}}$ cleavage upon TRAIL stimulation and thus, no caspase- 8 cleavage can be detected. Surprisingly, more 
cFlip $_{\mathrm{L}}$ was recruited to the TRAIL-DISC after treatment with Bortezomib [figure 21]. However, despite the increase in recruitment, cFlip is not able to prevent caspase- 8 activation. Here it can be shown for the first time, that cIAPs are recruited to the DISC in KK cells and that this recruitment is almost absent in Bortezomib treated cells. Importantly, cIAP levels are almost unchanged in the TCL highlighting this finding as a DISC specific event. It was then hypothesised that cIAP presence in the DISC might account for TRAIL-resistance and that Bortezomib might sensitise to TRAIL by abrogating their recruitment. However, overexpression of cIAPs did not abrogate apoptosis induction in KK cells treated with Bortezomib and TRAIL. This could either mean, cIAP overexpressing cells treated with Bortezomib are still not able to recruit cIAPs into the DISC despite overexpression and the question whether their recruitment accounts for TRAIL resistance is still not answered or that they are recruited but are not important for Bortezomib-mediated sensitisation to TRAIL. Besides being an inhibitor of the proteasome, many more functions of Bortezomib have been described, e.g. downregulation of $\mathrm{cFLIP}_{\mathrm{L}}$ and/or XIAP and reduction of $\mathrm{Bcl}-\mathrm{X}_{\mathrm{L}}$ phosphorylation (Frankel et al. 2000).

Since the overall expression levels of cIAPs are not markedly changed in Bortezomib treated cells, it might be possible that Bortezomib inhibits or downregulates an unknown protein, that is required to recruit cIAPs to the TRAIL-DISC. In order to follow this hypothesis, more experiments will be performed. An overexpression of cIAPs with subsequent immunoprecipitation of the TRAIL-DISC should reveal whether cIAP recruitment is reconstituted in Bortezomib treated cells or whether cIAPs are still not recruited despite overexpression.

Sensitisation to TRAIL-induced apoptosis by smac-mimetics has already been reported (Fulda et al. 2002), however, the exact mechanisms behind smac-mimetic induced sensitisation to TRAIL remains unkown. Furthermore, there is no proof yet, if at all and when at which level cIAPs exactly exert their anti-apoptotic function along the TRAIL signalling pathway. So far, the sensitisation effect of smac mimetics was believed to be solely due to inhibition of XIAP. Our laboratory has just recently observed presence of cIAPs in the DISC (unpublished data). However their exact localisation at the DISC remains to be investigated.

Although, TRAIL-R levels are up-regulated after treatment with Bortezomib [figure 18.1], this has not been shown to be essential for TRAIL-sensitisation mediated by Bortezomib (Ganten et al. 2005). In order to determine the contribution that TRAIL-R up-regulation has on the sensitisation effect, a series of so called "wash-kill" experiments can be performed (Ganten et al. 2005; Koschny et al. 2007c). If the TRAIL-R up-regulation by Bortezomib was 
necessary, then the binding of additional TRAIL to newly surface-expressed TRAIL-receptors would be inevitable for the sensitisation effect which did not seem to be the case.

In summary it can be stated, that the Bortezomib-mediated sensitisation to TRAIL-induced apoptosis might act at the level of DISC formation via a more efficient recruitment of FADD and caspase- 8 and possibly a downregulation of cIAPs.

\section{4. cIAPs are recruited into the DISC independently of FADD}

In order to define at which level of the DISC cIAPs might be recruited, DKO4 cells stably expressing dnFADD were used [figure 21]. Interestingly, dnFADD can not be recruited to the DISC in these cells. However, recruitment of cIAPs into the DISC is unchanged in dnFADD expressing DKO4 cells. This means that cIAPs must be recruited to the TRAIL receptor upstream of the DED of FADD or most likely independent of FADD. However, it might be the case, that dnFADD is not recognised by our antibody when it is recruited into the DISC where it changes its confirmation in order to be able to bind to the intracellular domain of the TRAIL-Receptors. Furthermore, dnFADD could be recruited into the DISC with different kinetics and as the IP only shows one timepoint it might have been missed. Furthermore, unstimulated receptors of DKO4 cells already show a weak presence of cIAPs, also suggesting that cIAPs directly bind to TRAIL-Rs.

Currently, there is nothing known about cIAPs at the CD95L-or TRAIL-DISC. However, in the TNF-RSC cIAPs have been shown to be present since the mid 1990s (Rothe et al. 1995). Binding of TNF- $\alpha$ to TNF-R1 leads to the formation of an intracellular multi-protein complex: the TNF-Receptor signalling complex (TNF-RSC). First, the TNF-R1 associated death domain (TRADD) binds to the intracellular DD of the TNF-R1 (Hsu et al. 1995) which then serves as a assembly platform for binding of TNF-receptor associated factor 2 (TRAF2) and TRAF5, as well as RIP1 (Hsu et al. 1996; Tsao et al. 2000). In addition, cIAP-1 and cIAP-2 are recruited to TRAF2 via their BIR1 domain to form part of the TNF-RSC (Shu et al. 1996; Vince et al. 2007; Varfolomeev et al. 2008). Since TRAF2 as well as cIAP-1 and-2 both contain a RING domain they have been described to be involved in the attachment of ubiquitin chains to RIP1. As interesting future experiments cIAP RING mutants can be created as tools in order to find out whether the ubiquitin ligase of cIAPs might be required for their recruitment to the DISC. Furthermore, as recruitment of cIAPs in the TNF-RSC is dependent on their BIR1 domain, it would be interesting to additionally create a BIR1 mutant 
in order identify its importance for DISC recruitment. Moreover, differences in the recruitment of cIAP to either TRAIL-R1,-R2, and/or -R4 would be interesting to follow up on.

\subsection{Sensitisation to TRAIL-induced apoptosis by PIK75 or Smac83}

Subtoxic concentrations of PIK75 and Smac83 were used for sensitisation experiments in PEA-1, PEA-2 and KK cells. PEA-1 and PEA-2 cells were potently sensitised to TRAIL-induced apoptosis, whereas the effect in KK cells was less pronounced [figure 16].

It has long been known that the PI3K/Akt pathway is vital to growth and survival of cancer cells (reviewed in Engelman 2009) and is one of the most frequently altered pathways in cancers. Therefore, drugs targeting the PI3K/Akt pathway have rapidly entered clinical trials. Tumour cells that harbour mutations in the PI3K/Akt pathway have been shown to be relatively TRAIL resistant (Samuels et al. 2005) however, inhibition of the PI3K/Akt pathway can sensitise to TRAIL-induced apoptosis in a variety of different tumour cells.

PIK3CA, which encodes for the p1 $10 \alpha$ catalytic subunit of PI3K is particularly often mutated in cancers. For instance, 32\% of colorectal cancers harbour PIK3CA mutations (Samuels et al. 2004). Ovarian cancers have also been described to specifically harbour PI3K-p110 $\alpha$ mutations, which was the reason for us to choose PIK75 as an inhibitor that specifically targets the p110 $\alpha$ isoform of PI3K and not a pan-PI3K inhibitor. Furthermore, the concern has been raised that complete inhibition of all PI3K isoforms or Akt might be much more toxic to patients (Engelman 2009) than inhibition of only one isoform.

So far, for epithelial ovarian cancer, PIK3CA mutations have mostly been described to occur in clear cell and endometrioid ovarian cancers. However, amplifications of PIK3CA were found to be frequent in all TP53 mutant tumours and also shown to decrease the likelihood of complete remission, additionally conveying platinum resistance (Iwona et al. 2009).

Since PEA-1 and PEA-2 cells are both high grade serous ovarian cancer cell lines that in over 90\% harbour TP53 mutations they might comprise an amplification of the PIK3CA which has been reported to occur in $13,3 \%$ to $18 \%$ of high-grade ovarian carcinomas (Nakayama et al. 2006). Both were sensitised to TRAIL by PI3K alpha inhibition suggesting that at least PI3K alpha plays a crucial role in these cells for TRAIL resistance. 
The PI3K inhibitors that are currently used mostly target all isoforms of PI3K. LY294002 is such an inhibitor and has been shown to sensitise human leukemic cells to TRAIL-induced apoptosis (Martelli et al. 2003). However, used in a mouse xeno-transplant model of ovarian cancer LY294002 inhibited tumour growth and ascites accumulation but also showed indication of toxicity as two out of twelve mice in the treatment group died (Hu et al. 2000). We have shown for the first time that the PI3K inhibitor PIK75 that specifically targets the $\alpha$ isoform of PI3K sensitises to TRAIL-induced apoptosis (K.Papenfuss unpublished data). Treatment of PEA-1 and PEA-2 cells with PIK75 up to 24h [figure 18.2], shows a potent downregulation of $\mathrm{CFLIP}_{\mathrm{L}}$ and $\mathrm{CFLIP}_{\mathrm{S}}$ enhancing caspase-8 activation at the DISC which has been reported before, upon broad spectrum inhibition of the PI3K pathway (Panka et al. 2001; Bortul et al. 2003; Kang et al. 2004). The downregulation of molecules belonging to the IAP family e.g. Survivin, cIAP-1, cIAP-2, XIAP has been shown upon inhibition of the PI3K pathway (Kim et al. 2004; Tang et al. 2009) and indeed downregulation of cIAP-2 and XIAP also correlated with the sensitisation effect to TRAIL-induced apoptosis observed in ovarian cancer cells [figure 19.2]. cIAP-1 was not affected. However, even if so far cIAP-1 and cIAP2 are mostly reported to act as one entity it should be considered that they might also have distinct functions.

In the PIK75 only kinetic where a pan cIAP-1/-2 Ab was used, only a minor downregulation of total cIAP levels could be detected. Without the application of antibodies that are able to differenciate between the different cIAPs the very potent effect of cIAP-2 could have been easily missed. Full cleavage of caspases indicated an activation of the apoptotic pathway upon co-treatment of PIK75 and TRAIL in PEA-1 cells.

As a further experiment, knock down of these proteins by small interfering RNA can be performed in order to determine whether all the regulated proteins cFLIP, XIAP and cIAP-2 are decisive factors for PIK75 mediated sensitisation to TRAIL-induced apoptosis. Furthermore, inhibition of the PI3K pathway has been correlated to upregulation of TRAILR1 and TRAIL-R2 (Tazzari et al. 2008). However, as already discussed for the sensitisation to TRAIL via Bortezomib, the upregulation of TRAIL-Rs has been observed very often upon treatment with different small molecule inhibitors but is mostly not decisive for the sensitisation effect.

Smac mimetics were recently found to be a promising candidate for cancer therapy, as they have been shown to lead to a complete eradication of established intracranial malignant 
gliomas and survival of mice in a xenograft model in vivo if combined with TRAIL. No toxicity to the surrounding normal brain tissue could be detected (Fulda et al. 2002). Smac mimetics have also been shown to synergise with Bortezomib and sensitise melanoma cell lines to TRAIL-induced apoptosis (Lecis et al. 2010). However, the combination treatment of Bortezomib, Smac83 and TRAIL in our system only led to an additional increase in cell death by approximately $0-10 \%$ in the cells (data not shown), which was regarded as minor when considering the increase of toxicity in multi drug combinations.

Cancer cells frequently overexpress IAPs resulting in expression levels that are much higher than in normal cells allowing the cancer to survive and progress. Smac mimetics were initially designed to inhibit XIAP but where then found to also lead to degradation of cIAPs (Varfolomeev et al. 2007; Vince et al. 2007). Smac mimetics are promising anticancer therapeutics that have been reported to induce apoptosis as a single agents or act as sensitisers to TRAIL-induced apoptosis in various cancer cell lines (Li L et al. 2004; Bockbrader et al. 2005; Chen et al. 2009; Dai et al. 2009). Recent studies showed, that both cIAPs and XIAP need to be concurrently removed in order to efficiently induce apoptosis in cancer cells by smac mimetics or small interfering RNA ( $\mathrm{Lu}$ et al. 2008). Thus both, cIAPs and XIAP are important cellular targets for smac mimetics.

Smac mimetics have just recently been shown to sensitise even resistant cells to TRAILinduced apoptosis by bypassing the Bcl-2 block at the mitochondria which prevents the release of SMAC from mitochondria into the cytosol. Furthermore, they sensitise by antagonising XIAP (Fulda et al. 2002).

S. Fulda and colleagues showed in their intracranial malignant glioma xenograft model that mice that were intracranially challenged with malignant glioma cells and treated with smac peptide and TRAIL did not develop any clinical symptoms after 70 days, whereas the average survival time of control mice was 30 days after tumour challenge. Importantly, cytotoxic effects on normal brain tissue upon co-injection of TRAIL and smac peptides were absent. Interestingly, recently it was published that the smac mimetic JP1584 can reduce TRAILinduced metastatic behaviour in cholangiocarcinoma supporting the emerging concept that IAPs are prometastatic and represent targets for anti-metastatic therapies (Fingas et al. 2010). In PEA-1 and PEA-2 cells two different smac mimetic compounds were tested. However, they both induced complete degradation of cIAP-1 and cIAP-2 after no longer than one hour 
treatment. Interestingly, they also both lead to a marked up-regulation of $\mathrm{cFLIP}_{\mathrm{S}}$ and a slight up-regulation of $\mathrm{cFLIP}_{\mathrm{L}}$ in these cells.

One could assume, that co-treatment of smac mimetics and PIK75 would synergise in sensitising tumour cells to TRAIL-induced apoptosis, however, the upregulation of cFLIP by smac mimetics can not be overcome by PIK75 and thus, does not further enhance killing in ovarian tumour cell lines and primary ovarian tumour samples (data not shown). The sensitisation effect that occurs, even though cFLIP is upregulated upon smac-mimetic treatment or PIK75, smac-mimetic co-treatment might be due to a role of cIAPs at the DISC, which might be able to transmit the death signal although cFLIP is upregulated.

Clinically, resistance of apoptosis is a major cause of primary or acquired non-responsiveness of cancer cells leading to treatment failure. However, here it could demonstrated that different small molecule inhibitors can efficiently sensitise chemoresistant ovarian cancer cells to TRAIL-induced apoptosis which might initiate new treatment options for platinum resistant ovarian cancer patients.

\subsection{Primary ovarian cancer cells}

Based on the results obtained in ovarian cancer cell lines and with the long-term idea of establishing a new clinical trial for ovarian cancer with TRAIL, the just identified treatment options were ought to be tested on primary ovarian cancer cells as well.

Immortalised cell lines undergo many genetic changes during their establishment which limits our ability to translate results obtained from cell lines directly to actual ovarian disease in the clinic. Thus, primary tumour cultures may provide a better substrate for in vitro studies and results obtained from primary cells are much more relevant for clinical application.

However, a reliable and effective technique for the isolation of ovarian cancer cells from ascitic fluid has not yet been fully established and thus, has limited the ability to work with primary tumour cells. Many protocols of purifying tumour cells rely on filtration and density gradients (Hirte et al. 1992; Shepherd et al. 2006). These techniques however, do not selectively isolate malignant cells and thus, can have high rates of contamination with benign cells such as mesothelial cells, monocytes and fibroblasts which, like cancer cells, usually also adhere to cell culture plastic. Other CD45-positive cells that do not adhere might be 
washed away during the first set of medium changes but as cancer cells can survive anchorage-independently within the ascitic fluid, a fraction of cancer cells that does not adhere to tissue culture plastic might be washed away together with CD45-positive haematopoietic cells. Furthermore, tumour cell density differs significantly, so that many reports could only demonstrate a tumour purity of $42 \%$ as tumour cells were lost in the gradient (Castells et al. 1998).

EpCAM (Ber-EP4, HEA-125, CD326), provides an optimal antigenic target for the isolation of malignant cells of epithelial origin, because it is expressed on $80 \%$ of epithelial tumour cells (Latza et al. 1990; Went et al. 2004) and does not cross react with mesothelial cells, fibroblasts, and other non-epithelial cells like CD45-positive haematopoietic cells e.g. monocytes, that often contaminate tumour cell purifications.

For instance, one study used the Dynabeads CELLection kit with EpCAM beads as a method of choice for isolating ovarian cancer cells from ascitic fluid (Kielhorn et al. 2002). These beads contain the same antibody as the Epithelial Enrich beads that were used in this thesis, but there they are attached to the beads via a DNA linker, which can be cut using Dnase to facilitate release of the cells. However, the release of the cells is not $100 \%$ effective (personal communication Invitrogen) and not recommended if many cells need to be used for downstream analysis. Furthermore, cell viability might be affected.

However, in this study the isolation was only used for screening purposes rather than using the cells for any downstream analysis which might have been due to reduced cell viability. (Kielhorn et al. 2002). As it could be proven before, that EpCAM Dynabeads neither alter the outcome of the assays that were employed nor changed morphology or viability of IGROV-1 cells they were used to purify primary ovarian cancer cells [figure 22]. Furthermore, the morphology of primary cells was frequently checked under the microscope and it can be stated, that cell viability was not decreased after co-incubating with EpCAM Dynabeads.

In addition, Kielhorn and colleges determined purity only by morphology of cancer cells and E-Cadherin staining. E-Cadherin expression has been reported to be significantly increased in cancerous ovaries as compared to normal tissue (Chhieng et al. 2000; Ansenberger et al. 2009) however, cancer cells that have undergone Epithelial-Mesenchymal-Transition (EMT) (reviewed in (Visvader and Lindeman 2008)) downregulate epithelial markers such as Ecadherin expression on their surface (Blechschmidt et al. 2008; Klopp et al. 2010). Especially ovarian cancer cells that can survive anchorage independently in the ascitic fluid are thought to have undergone an EMT since anchorage-independent growth is one of the features of 
EMT (Lee et al. 2008). These cells might therefore not express E-cadherin on their surface. Using the tumorigenic cell line RERF-LC-Al which is negative for the expression of Ecadherin, it was shown, that forced expression of E-cadherin inhibited ovarian metastasis (Kuwabara et al. 2008). Since the accumulation of ascites is mostly a sign of aggressive and late stage disease we conclude that E-cadherin is not a suitable marker for primary ovarian cancer cells isolated from ascites fluid.

Most of the primary ovarian cancer cells were obtained from patients that presented with recurrent, chemotherapy resistant ovarian cancer. Despite their resistance to chemotherapy, they could effectively be sensitised to TRAIL with Bortezomib, Smac83 or PIK75. Co-incubation of cisplatin and TRAIL could also sensitise even chemotherapy resistant ovarian cancer cells to TRAIL-induced apoptosis. The observation that cisplatin synergises with TRAIL to induce apoptosis in otherwise cisplatin resistant ovarian cancer cells has been made before (Cuello et al. 2001). Cisplatin and TRAIL are already applied in Phase I and II clinical trials for advanced solid tumours and might also be an option for patients with platinum resistant ovarian cancer.

The difficulty that arose after having treated the first sets of isolated ovarian cancer cells with TRAIL was that the EpCAM negative population that should have only contained normal cells was less but still significantly susceptible to treatment with TRAIL with or without the addition of small molecule inhibitors as compared to EpCAM positive cancer cells. It was therefore important to investigate which cells were present in the EpCAM negative fraction. Even though it has already been proven in vivo that TRAIL specifically induces apoptosis in tumour cells (Walczak et al. 1999) without harming normal cells, it was thought to be important to verify these findings for these specific combinational treatments in primary ovarian cancer cells. In order to obtain a cell population that contained only normal cells, an additional purification step was added to the original protocol. CD45 Dynabeads ${ }^{\circledR}$ were used with the aim to define a completely normal cell population that would serve as a control group. Even though the length of the purification protocol has been slightly extended, a clear differentiation of cancer cells and non-cancer cells can now be ensured. Furthermore, in most of the samples, CD45 positive cells, especially monocytes comprised the main population of cells in the ascitic fluid and even though Fc-Receptors were blocked prior to Dynabeads ${ }^{\circledR}$ isolation and FACS staining, the chance of unspecific binding of CD45 positive cells to EpCAM Dynabeads ${ }^{\circledR}$, could now be further decreased by removing them in advance. 
It could be shown in an exemplary Flow Cytometry dot plot that $\sim 83 \%$ of CD45 positive cells were pulled out by CD45 Dynabeads, leading to an enrichment of the EpCAM population to $\sim 73 \%$. However, it has to be considered that because the initial EpCAM population can vary tremendously among patients (3\%-18\%) the EpCAM enrichment might also vary accordingly. 
6. Summary and Outlook 


\section{Summary and Outlook:}

Ovarian carcinoma is the most deadly gynaecological malignancy and the fifth leading cause of carcinoma death in American women. Approximately $70 \%$ of women with ovarian cancer present with advanced stage disease with either regional or distant metastases at the time of diagnosis. Although the 5-year survival data are $88 \%$ and $59 \%$ in FIGO stages I and II, respectively, the more advanced stages III and IV have a survival of approximately only $30 \%$ and $18 \%$, respectively. The greatest challenge in the treatment of women with ovarian carcinoma is the acquisition of chemotherapy resistance which can occur even following complete clinical response.

The results of this study suggest that the combination of TRAIL with PIK75, Smac83/59 or Bortezomib could be promising new options for ovarian cancer therapy, in particular for platinum-resistant recurrent ovarian cancer.

Taken together it was shown that the majority of ovarian carcinoma cell lines were TRAILresistant. TRAIL apoptosis sensitivity did not correlate with the histopathological subtype of the ovarian carcinoma. The application of sub-toxic doses of different small molecule inhibitors led to sensitisation of most of the cell lines. For instance, KK, PEA-1 and PEA-2 cells could be sensitised to TRAIL-induced apoptosis with Bortezomib, PIK75 and Smac59/83. TOV-112D cells displayed an exemption as they could not be sensitised to TRAIL-induced apoptosis with any of the applied small molecule-inhibitors.

The form of cell death caused by the combination of TRAIL and the sensitising agent could subsequently be classified as apoptosis by measuring the sub-diploid DNA content and complete blockage of apoptosis following treatment of cells with the caspase-inhibitor Q-VD-OPh.

Molecules potentially involved in TRAIL-sensitisation by Bortezomib were identified by WB. Staining of TRAIL-R1 and TRAIL-R2 showed an upregulation predominantly of TRAIL-R1 however, this only marginally contributed to the observed sensitisation effect.

Treatment of the isogenic matched pair PEA-1/2, of which PEA-2 cells display platinum resistance and therefore are able to imitate a situation that is more realistic to the clinical setting, were both treated with PIK75 and Smac59/83. Application of PIK75 led to massive downregulation of $\mathrm{cFLIP}_{\mathrm{L}}, \mathrm{cFLIP}_{\mathrm{S}}$, XIAP and, to a minor degree, of cIAPs. Smac59/83 on the one side resulted in an immediate downregulation of cIAP-1 as well as cIAP-2. On the other 
hand both SMAC mimetics led to an upregulation of FLIP $P_{S}$ and also slightly upregulated $\mathrm{cFLIP}_{\mathrm{L}}$. Upon TRAIL application, cells that were pretreated with Bortezomib, PIK75 or Smac59/83, respectively, exhibited strong caspase and PARP cleavage as sign of apoptosis induction.

The subsequent successful production of recombinant moTAP-TRAIL could be used to perform immuno-precipitations of the TRAIL-DISC in KK cells. Besides confirming the published data with respect to enhanced DISC formation upon Bortezomib treatment, new results were obtained demonstrating the recruitment of cIAPs to the TRAL-DISC, an effect that was fully abrogated by pretreatment with Bortezomib. As cIAP levels in the TCL remain almost unchanged, the effect can be classified as DISC-specific. In order to further elucidate the localisation of cIAPs at the TRAIL-DISC, DKO4 cells overexpressing a dominant negative mutant of FADD were used. Interestingly, the recruitment of cIAPs to the DISC remained unchanged in dnFADD cells pointing towards the recruitment of cIAPs to TRAIL receptors being independent of FADD.

Besides the work with cell lines a major result of this thesis is the establishment of the isolation of ovarian cancer cells from ascitic fluid. As primary material is rare, cell lines appeared to be a very useful tool to study the biochemical effects that different drugs have on the TRAIL signalling pathway. However, when it comes to clinical significance, primary material displays a much more realistic setting.

EpCAM-Dynabeads ${ }^{\circledR}$ were used for the isolation of ovarian cancer cells. Prior to working with patient material the EpCAM expressing cell line IGROV-1 was used for optimisation of the protocol. It could be shown that IGROV-1 cells isolated with Dynabeads ${ }^{\circledR}$ retain their normal morphology, establish normal cell-to-cell contacts and do not behave differently in viability or proliferation assays as compared to control IGROV-1 cells.

The subsequent isolation of EpCAM-positive ovarian cancer cells from patient's ascites showed that primary ovarian cancer cells, even when obtained from patients with recurrent cisplatin-resistant carcinomas, can be sensitised to TRAIL-induced apoptosis. However, as the EpCAM-negative population seemed to be rather a heterogeneous population of EpCAMnegative cancer and non-cancer cells, no clear statement concerning the toxicity of the different drugs could be made. In order to be able to clearly define a healthy cell population, CD45 Dynabeads ${ }^{\circledR}$ were introduced into the purification protocol prior to the EpCAM isolation. With the application of these additional Dynabeads ${ }^{\circledR}$ clear differentiation can be 
made between cancer and non-cancer cells confirming once again the specificity of TRAILinduced apoptosis to cancer cells leaving normal, CD45-positive cells largely unaffected.

Having determined many factors that are regulated upon treatment with PIK75, Smac83 and Bortezomib such as XIAP, cFLIP and cIAP-1/2 it would be most interesting to define novel factors that are also regulated upon stimulation with the different drug combinations. In order to achieve this, total RNA was isolated from freshly purified ovarian cancer cells. These cells were previously treated with PIK75, Smac83 or Bortezomib. Deep RNA sequencing of these samples (also called genome-wide expression profiling or transcriptomics) is planned as a future experiment. RNA sequencing aims to catalogue the complete set of RNA transcripts produced by the genome, including mRNAs, non-coding RNAs and small RNAs. It can be used to detect novel transcripts and quantify the changing expression levels of each transcript under different disease conditions and/or upon treatment with different small molecule inhibitors. This could provide new additional insight into transcriptional regulation of different genes upon stimulation with PIK75, Bortezomib, Smac83 and other drugs.

Furthermore, deep RNA sequencing could have prognostic value by determining the transcriptional levels of different target genes in the transcriptome of patients and could be used to identify more tailored therapy options for each single patient in order to treat them accordingly. 


\section{List of Abbreviations}

\begin{tabular}{|c|c|}
\hline Apaf-1 & Adaptor protein apoptotic peptidase activating factor 1 \\
\hline ATP & Adenosintriphosphat \\
\hline Bad & Bcl-2-associated death promoter induced by cytokine deprivation \\
\hline Bak & Bcl-2 homologous antagonist/killer \\
\hline Bax & Bcl-2-associated X protein \\
\hline BCA & Bicinchoninic acid \\
\hline Bel & B cell lymphoma \\
\hline Bcl-XL & Bcl-extra large \\
\hline BH & BH3-homology \\
\hline Bid & BH3-interacting domain death agonist \\
\hline Bik & Bcl-2 interacting killer induced by endoplasmic reticulum stress \\
\hline Bim & Bcl-2-like protein 11 \\
\hline BIR & Baculovirus IAP repeat domain \\
\hline Bp & Base Pair \\
\hline BRAF & B-Raf proto-oncogenen serine-threonine/proteinkinase \\
\hline CAD & Caspase-activated Dnase \\
\hline CARD & Caspase recruitment domain \\
\hline Caspase & Cysteine-dependent, aspartate-specific acid proteases \\
\hline cFLIP & Cellular FLICE-inhibitory protein \\
\hline CML & Chronic myeloid leukaemia \\
\hline DcR & Decoy Receptor \\
\hline DD & Death domain \\
\hline DED & Death effector domain \\
\hline DIABLO & direct IAP binding protein with low isoelectric point \\
\hline DISC & Death-inducing signalling complex \\
\hline DMEM & Dulbecco's modified Eagle's medium \\
\hline DMSO & Dimethylsulfoxid \\
\hline DR & Death Receptor \\
\hline E.coli & Escherichia coli \\
\hline EDTA & Ethylendiaminetetraacetic acid \\
\hline EGF & Epidermal growth factor \\
\hline EPCAM & Epithelial-specific cell adhesion molecule \\
\hline ERK & Extracellular signal-regulated kinase \\
\hline FADD & Fas-associated protein with death domain \\
\hline FCS & Fetal Calf serum \\
\hline 5-FU & 5-Fluorouracil \\
\hline GI & Gastrointestinal \\
\hline GPI & Glycosylphophatidylinositol \\
\hline HRP & Horse-raddish peroxidase \\
\hline IAP & Inhibitor of apoptosis \\
\hline IKK & I $\kappa$ B-kinase \\
\hline Iz & Isoleucine-zipper \\
\hline JNK & C-Jun N-terminal kinase \\
\hline KRAS & Kirsten rat sarcoma viral oncogene homolog \\
\hline LS & Large subunit \\
\hline MAPK & Mitogen-activated kinases \\
\hline Mcl-1 & Myeloid cell leukemia-1 protein \\
\hline
\end{tabular}




\begin{tabular}{ll} 
MCMV & Murine Cytomegalovirus \\
MTT & Methoxy Tetrazolium salt \\
MOMP & Mitochondrial outer membrane permeabilisation \\
moTAP & Modified Tandem Affinity Purification \\
MTT & 3-(4,5-Dimethylthiazol-2-yl)-2,5-diphenyltetrazolium bromide \\
NF-kB & Nuclear factor 'kappa-light-chain-enhancer' of activated B-cells \\
NHL & Non-Hodgkin lymphoma \\
NOXA & Phorbol-12-myristate-13-acetate-induced protein 1 \\
NSCLC & Non-small cell lung cancer \\
OPG & Osteoprotegerin \\
PARP & Poly (ADP-ribose) polymerase \\
PI3K & Phosphoinositide 3-kinase \\
PLAD & Pre-ligand assembly domain \\
PTEN & Phosphatise and tensin homolog \\
PUMA & P53 upregulated modulator of apoptosis \\
RANK & Receptor activator of NF-kB \\
RIP1 & Receptor Interacting Protein 1 \\
RING & Really interesting new gene \\
RPMI & Roswell Park memorial Institute \\
RT & Room temperature \\
RTK & Receptor tyrosine kinase \\
SD & Standard deviation \\
SDS & Sodium dodecyl sulphate \\
SDS-PAGE & Sodium dodecyl sulphate polyacrylamide gel electrophoresis \\
SL & Small subunit \\
SMAC & Second mitochondria-derived activator of caspases \\
TACE & Tumour necrosis factor- $\alpha$-converting enzyme \\
tBid & Truncated Bid \\
TCL & Total cell lyates \\
TM & Transmembrane \\
TNF & Tumour necrosis factor \\
TRAIL & TNF-related apoptosis-inducing ligand \\
TRAIL-R & TNF-related apoptosis-inducing ligand receptor \\
UBA & Ub associated \\
Ub & Ubiquitin \\
Wt & Wild type \\
XIAP & X-linked inhitor apoptosis protein \\
\hline
\end{tabular}


8. References 


\section{References}

Acehan, D., X. Jiang, D. G. Morgan, J. E. Heuser, X. Wang and C. W. Akey (2002). "Threedimensional structure of the apoptosome: implications for assembly, procaspase- 9 binding, and activation." Mol Cell 9(2): 423-32.

Adams, J. (2001). "Proteasome inhibition in cancer: development of PS-341." Semin Oncol 28(6): 613-9.

Adams, J., V. J. Palombella, E. A. Sausville, J. Johnson, A. Destree, D. D. Lazarus, J. Maas, C. S. Pien, S. Prakash and P. J. Elliott (1999). "Proteasome inhibitors: a novel class of potent and effective antitumor agents." Cancer Res 59(11): 2615-22.

Adams, K. W. and G. M. Cooper (2007). "Rapid turnover of mcl-1 couples translation to cell survival and apoptosis." J Biol Chem 282(9): 6192-200.

Ahmed, A. A., D. Etemadmoghadam, J. Temple, A. G. Lynch, M. Riad, R. Sharma, C. Stewart, S. Fereday, C. Caldas, A. Defazio, D. Bowtell and J. D. Brenton "Driver mutations in TP53 are ubiquitous in high grade serous carcinoma of the ovary." J Pathol 221(1): 49-56.

Al-Hajj, M., M. S. Wicha, A. Benito-Hernandez, S. J. Morrison and M. F. Clarke (2003). "Prospective identification of tumorigenic breast cancer cells." Proc Natl Acad Sci U S A 100(7): 3983-8.

Andrade, R., L. Crisol, R. Prado, M. D. Boyano, J. Arluzea and J. Arechaga (2010). "Plasma membrane and nuclear envelope integrity during the blebbing stage of apoptosis: a time-lapse study." Biol Cell 102(1): 25-35.

Ansenberger, K., Y. Zhuge, J. A. Lagman, C. Richards, A. Barua, J. M. Bahr and D. B. Hales (2009). "E-cadherin expression in ovarian cancer in the laying hen, Gallus domesticus, compared to human ovarian cancer." Gynecol Oncol 113(3): 362-9.

Ashkenazi, A. and V. M. Dixit (1998). "Death receptors: signaling and modulation." Science 281(5381): 1305-8.

Ashkenazi, A., R. C. Pai, S. Fong, S. Leung, D. A. Lawrence, S. A. Marsters, C. Blackie, L. Chang, A. E. McMurtrey, A. Hebert, L. DeForge, I. L. Koumenis, D. Lewis, L. Harris, J. Bussiere, H. Koeppen, Z. Shahrokh and R. H. Schwall (1999). "Safety and antitumor activity of recombinant soluble Apo2 ligand." J Clin Invest 104(2): 155-62.

Baeuerle, P. A. and O. Gires (2007). "EpCAM (CD326) finding its role in cancer." Br J Cancer 96(3): 417-23.

Barnhart, B. C., E. C. Alappat and M. E. Peter (2003). "The CD95 type I/type II model." Semin Immunol 15(3): 185-93.

Bhattacharyya, A., U. S. Ear, B. H. Koller, R. R. Weichselbaum and D. K. Bishop (2000). "The breast cancer susceptibility gene BRCA1 is required for subnuclear assembly of Rad51 and survival following treatment with the DNA cross-linking agent cisplatin." $\underline{J}$ Biol Chem 275(31): 23899-903.

Blechschmidt, K., S. Sassen, B. Schmalfeldt, T. Schuster, H. Hofler and K. F. Becker (2008). "The E-cadherin repressor Snail is associated with lower overall survival of ovarian cancer patients." Br J Cancer 98(2): 489-95.

Boatright, K. M. and G. S. Salvesen (2003). "Mechanisms of caspase activation." Curr Opin Cell Biol 15(6): 725-31.

Boatright, K. M., C. Deis, J. B. Denault, D. P. Sutherlin and G. S. Salvesen (2004). "Activation of caspases-8 and -10 by FLIP(L)." Biochem J 382(Pt 2): 651-7. 
Bockbrader, K. M., M. Tan and Y. Sun (2005). "A small molecule Smac-mimic compound induces apoptosis and sensitizes TRAIL- and etoposide-induced apoptosis in breast cancer cells." Oncogene 24(49): 7381-8.

Bortul, R., P. L. Tazzari, A. Cappellini, G. Tabellini, A. M. Billi, R. Bareggi, L. Manzoli, L. Cocco and A. M. Martelli (2003). "Constitutively active Akt1 protects HL60 leukemia cells from TRAIL-induced apoptosis through a mechanism involving NF-kappaB activation and cFLIP(L) up-regulation." Leukemia 17(2): 379-89.

Burges, A., P. Wimberger, C. Kumper, V. Gorbounova, H. Sommer, B. Schmalfeldt, J. Pfisterer, M. Lichinitser, A. Makhson, V. Moiseyenko, A. Lahr, E. Schulze, M. Jager, M. A. Strohlein, M. M. Heiss, T. Gottwald, H. Lindhofer and R. Kimmig (2007). "Effective relief of malignant ascites in patients with advanced ovarian cancer by a trifunctional anti-EpCAM x anti-CD3 antibody: a phase I/II study." Clin Cancer Res 13(13): 3899-905.

Carpenter, G. and M. Red Brewer (2009). "EpCAM: another surface-to-nucleus missile." Cancer Cell 15(3): 165-6.

Caserta, T. M., A. N. Smith, A. D. Gultice, M. A. Reedy and T. L. Brown (2003). "Q-VD$\mathrm{OPh}$, a broad spectrum caspase inhibitor with potent antiapoptotic properties." Apoptosis 8(4): 345-52.

Castells, A., L. Boix, X. Bessa, L. Gargallo and J. M. Pique (1998). "Detection of colonic cells in peripheral blood of colorectal cancer patients by means of reverse transcriptase and polymerase chain reaction." Br J Cancer 78(10): 1368-72.

Chan, F. K. (2007). "Three is better than one: pre-ligand receptor assembly in the regulation of TNF receptor signaling." Cytokine 37(2): 101-7.

Chang, D. W., Z. Xing, Y. Pan, A. Algeciras-Schimnich, B. C. Barnhart, S. Yaish-Ohad, M. E. Peter and X. Yang (2002). "c-FLIP(L) is a dual function regulator for caspase-8 activation and CD95-mediated apoptosis." EMBO J 21(14): 3704-14.

Chen, D. J. and S. Huerta (2009). "Smac mimetics as new cancer therapeutics." Anticancer Drugs 20(8): 646-58.

Chhieng, D. C., H. Yee, J. F. Cangiarella, W. F. Symmans and J. M. Cohen (2000). "Use of E-cadherin and CD44 aids in the differentiation between reactive mesothelial cells and carcinoma cells in pelvic washings." Cancer 90(5): 299-306.

Cho, K. R. (2009). "Ovarian cancer update: lessons from morphology, molecules, and mice." Arch Pathol Lab Med 133(11): 1775-81.

Cho, K. R. and M. Shih Ie (2009). "Ovarian cancer." Annu Rev Pathol 4: 287-313.

Clancy, L., K. Mruk, K. Archer, M. Woelfel, J. Mongkolsapaya, G. Screaton, M. J. Lenardo and F. K. Chan (2005). "Preligand assembly domain-mediated ligand-independent association between TRAIL receptor 4 (TR4) and TR2 regulates TRAIL-induced apoptosis." Proc Natl Acad Sci U S A 102(50): 18099-104.

Clarke, P. and K. L. Tyler (2007). "Down-regulation of cFLIP following reovirus infection sensitizes human ovarian cancer cells to TRAIL-induced apoptosis." Apoptosis 12(1): 211-23.

Cordier, S. M., K. Papenfuss and H. Walczak (2009). "From biochemical principles of apoptosis induction by TRAIL to application in tumour therapy." Results Probl Cell Differ 49: 115-43.

Cretney, E., K. Takeda, H. Yagita, M. Glaccum, J. J. Peschon and M. J. Smyth (2002). "Increased susceptibility to tumor initiation and metastasis in TNF-related apoptosisinducing ligand-deficient mice." J Immunol 168(3): 1356-61. 
Cretney, E., J. L. McQualter, N. Kayagaki, H. Yagita, C. C. Bernard, I. S. Grewal, A. Ashkenazi and M. J. Smyth (2005). "TNF-related apoptosis-inducing ligand (TRAIL)/Apo2L suppresses experimental autoimmune encephalomyelitis in mice." Immunol Cell Biol 83(5): 511-9.

Crotzer, D. R., C. C. Sun, R. L. Coleman, J. K. Wolf, C. F. Levenback and D. M. Gershenson (2007). "Lack of effective systemic therapy for recurrent clear cell carcinoma of the ovary." Gynecol Oncol 105(2): 404-8.

Cuello, M., S. A. Ettenberg, M. M. Nau and S. Lipkowitz (2001). "Synergistic induction of apoptosis by the combination of trail and chemotherapy in chemoresistant ovarian cancer cells." Gynecol Oncol 81(3): 380-90.

Curley, M. D., V. A. Therrien, C. L. Cummings, P. A. Sergent, C. R. Koulouris, A. M. Friel, D. J. Roberts, M. V. Seiden, D. T. Scadden, B. R. Rueda and R. Foster (2009). "CD133 expression defines a tumor initiating cell population in primary human ovarian cancer." Stem Cells 27(12): 2875-83.

Dai, Y., M. Liu, W. Tang, Y. Li, J. Lian, T. S. Lawrence and L. Xu (2009). "A Smac-mimetic sensitizes prostate cancer cells to TRAIL-induced apoptosis via modulating both IAPs and NF-kappaB." BMC Cancer 9: 392.

Degli-Esposti, M. A., P. J. Smolak, H. Walczak, J. Waugh, C. P. Huang, R. F. DuBose, R. G. Goodwin and C. A. Smith (1997a). "Cloning and characterization of TRAIL-R3, a novel member of the emerging TRAIL receptor family." J Exp Med 186(7): 1165-70.

Degli-Esposti, M. A., W. C. Dougall, P. J. Smolak, J. Y. Waugh, C. A. Smith and R. G. Goodwin (1997b). "The novel receptor TRAIL-R4 induces NF-kappaB and protects against TRAIL-mediated apoptosis, yet retains an incomplete death domain." Immunity 7(6): 813-20.

Degterev, A., J. Hitomi, M. Germscheid, I. L. Ch'en, O. Korkina, X. Teng, D. Abbott, G. D. Cuny, C. Yuan, G. Wagner, S. M. Hedrick, S. A. Gerber, A. Lugovskoy and J. Yuan (2008). "Identification of RIP1 kinase as a specific cellular target of necrostatins." Nat Chem Biol 4(5): 313-21.

Deininger, M. W. and B. J. Druker (2003). "Specific targeted therapy of chronic myelogenous leukemia with imatinib." Pharmacol Rev 55(3): 401-23.

Diaz-Arias, A. A., T. S. Loy, J. T. Bickel and R. K. Chapman (1993). "Utility of BER-EP4 in the diagnosis of adenocarcinoma in effusions: an immunocytochemical study of 232 cases." Diagn Cytopathol 9(5): 516-21.

Diehl, G. E., H. H. Yue, K. Hsieh, A. A. Kuang, M. Ho, L. A. Morici, L. L. Lenz, D. Cado, L. W. Riley and A. Winoto (2004). "TRAIL-R as a negative regulator of innate immune cell responses." Immunity 21(6): 877-89.

Dinh, P., P. Harnett, M. J. Piccart-Gebhart and A. Awada (2008). "New therapies for ovarian cancer: cytotoxics and molecularly targeted agents." Crit Rev Oncol Hematol 67(2): 103-12.

Druker, B. J., M. Talpaz, D. J. Resta, B. Peng, E. Buchdunger, J. M. Ford, N. B. Lydon, H. Kantarjian, R. Capdeville, S. Ohno-Jones and C. L. Sawyers (2001). "Efficacy and safety of a specific inhibitor of the BCR-ABL tyrosine kinase in chronic myeloid leukemia." N Engl J Med 344(14): 1031-7.

Dzieran, J., J. F. Beck and J. Sonnemann (2008). "Differential responsiveness of human hepatoma cells versus normal hepatocytes to TRAIL in combination with either histone deacetylase inhibitors or conventional cytostatics." Cancer Sci 99(8): 1685-92.

Eckelman, B. P., G. S. Salvesen and F. L. Scott (2006). "Human inhibitor of apoptosis proteins: why XIAP is the black sheep of the family." EMBO Rep 7(10): 988-94. 
Elliott, P. J. and J. S. Ross (2001). "The proteasome: a new target for novel drug therapies." Am J Clin Pathol 116(5): 637-46.

Elmore, S. (2007). "Apoptosis: a review of programmed cell death." Toxicol Pathol 35(4): 495-516.

Emery, J. G., P. McDonnell, M. B. Burke, K. C. Deen, S. Lyn, C. Silverman, E. Dul, E. R. Appelbaum, C. Eichman, R. DiPrinzio, R. A. Dodds, I. E. James, M. Rosenberg, J. C. Lee and P. R. Young (1998). "Osteoprotegerin is a receptor for the cytotoxic ligand TRAIL." J Biol Chem 273(23): 14363-7.

Engelman, J. A. (2009). "Targeting PI3K signalling in cancer: opportunities, challenges and limitations." Nat Rev Cancer 9(8): 550-62.

Feeley, K. M. and M. Wells (2001). "Precursor lesions of ovarian epithelial malignancy." Histopathology 38(2): 87-95.

Fingas, C. D., B. R. Blechacz, R. L. Smoot, M. E. Guicciardi, J. Mott, S. F. Bronk, N. W. Werneburg, A. E. Sirica and G. J. Gores (2010). "A smac mimetic reduces TNF related apoptosis inducing ligand (TRAIL)-induced invasion and metastasis of cholangiocarcinoma cells." Hepatology 52(2): 550-61.

Frankel, A., S. Man, P. Elliott, J. Adams and R. S. Kerbel (2000). "Lack of multicellular drug resistance observed in human ovarian and prostate carcinoma treated with the proteasome inhibitor PS-341." Clin Cancer Res 6(9): 3719-28.

Frese, S., F. Pirnia, D. Miescher, S. Krajewski, M. M. Borner, J. C. Reed and R. A. Schmid (2003). "PG490-mediated sensitization of lung cancer cells to Apo2L/TRAIL-induced apoptosis requires activation of ERK2." Oncogene 22(35): 5427-35.

Fricker, N., J. Beaudouin, P. Richter, R. Eils, P. H. Krammer and I. N. Lavrik (2010). "Model-based dissection of CD95 signaling dynamics reveals both a pro- and antiapoptotic role of c-FLIPL." J Cell Biol 190(3): 377-89.

Fulda, S., E. Meyer and K. M. Debatin (2002a). "Inhibition of TRAIL-induced apoptosis by Bcl-2 overexpression." Oncogene 21(15): 2283-94.

Fulda, S., W. Wick, M. Weller and K. M. Debatin (2002b). "Smac agonists sensitize for Apo2L/TRAIL- or anticancer drug-induced apoptosis and induce regression of malignant glioma in vivo." Nat Med 8(8): 808-15.

Fulda, S. and K. M. Debatin (2006). "Extrinsic versus intrinsic apoptosis pathways in anticancer chemotherapy." Oncogene 25(34): 4798-811.

Ganten, T. M., T. L. Haas, J. Sykora, H. Stahl, M. R. Sprick, S. C. Fas, A. Krueger, M. A. Weigand, A. Grosse-Wilde, W. Stremmel, P. H. Krammer and H. Walczak (2004). "Enhanced caspase- 8 recruitment to and activation at the DISC is critical for sensitisation of human hepatocellular carcinoma cells to TRAIL-induced apoptosis by chemotherapeutic drugs." Cell Death Differ 11 Suppl 1: S86-96.

Ganten, T. M., R. Koschny, T. L. Haas, J. Sykora, M. Li-Weber, K. Herzer and H. Walczak (2005). "Proteasome inhibition sensitizes hepatocellular carcinoma cells, but not human hepatocytes, to TRAIL." Hepatology 42(3): 588-97.

Ganten, T. M., R. Koschny, J. Sykora, H. Schulze-Bergkamen, P. Buchler, T. L. Haas, M. B. Schader, A. Untergasser, W. Stremmel and H. Walczak (2006). "Preclinical differentiation between apparently safe and potentially hepatotoxic applications of TRAIL either alone or in combination with chemotherapeutic drugs." $\underline{\text { Clin Cancer Res }}$ 12(8): 2640-6.

Geserick, P., C. Drewniok, M. Hupe, T. L. Haas, P. Diessenbacher, M. R. Sprick, M. P. Schon, F. Henkler, H. Gollnick, H. Walczak and M. Leverkus (2008). "Suppression of cFLIP is sufficient to sensitize human melanoma cells to TRAIL- and CD95Lmediated apoptosis." Oncogene 27(22): 3211-20. 
Gilks, C. B. (2010). "Molecular abnormalities in ovarian cancer subtypes other than highgrade serous carcinoma." J Oncol 2010: 740968.

Gonzalvez, F. and A. Ashkenazi (2010). "New insights into apoptosis signaling by Apo2L/TRAIL." Oncogene 29(34): 4752-65.

Greenlee, R. T., M. B. Hill-Harmon, T. Murray and M. Thun (2001). "Cancer statistics, 2001." CA Cancer J Clin 51(1): 15-36.

Grosse-Wilde, A. O. Voloshanenko, L.S. Bailey, G. M. Longton, U. Schaefer, A. I. Csernok, G. Schütz, E. F. Greiner, C. J. Kemp and Henning Walczak (2008). "TRAIL-R deficiency in mice enhances lymph node metastasis without affecting primary tumor development."J Clin Inv 118 (1):100-110.

Gyrd-Hansen, M. and P. Meier (2010). "IAPs: from caspase inhibitors to modulators of NFkappaB, inflammation and cancer." Nat Rev Cancer 10(8): 561-74.

Haas, T. L., C. H. Emmerich, B. Gerlach, A. C. Schmukle, S. M. Cordier, E. Rieser, R. Feltham, J. Vince, U. Warnken, T. Wenger, R. Koschny, D. Komander, J. Silke and H. Walczak (2009). "Recruitment of the linear ubiquitin chain assembly complex stabilizes the TNF-R1 signaling complex and is required for TNF-mediated gene induction." Mol Cell 36(5): 831-44.

Hanahan, D. and R. A. Weinberg (2000). "The hallmarks of cancer." Cell 100(1): 57-70.

Harper, N., S. N. Farrow, A. Kaptein, G. M. Cohen and M. MacFarlane (2001). "Modulation of tumor necrosis factor apoptosis-inducing ligand- induced NF-kappa B activation by inhibition of apical caspases." J Biol Chem 276(37): 34743-52.

Harper, N., M. A. Hughes, S. N. Farrow, G. M. Cohen and M. MacFarlane (2003). "Protein kinase $\mathrm{C}$ modulates tumor necrosis factor-related apoptosis-inducing ligand-induced apoptosis by targeting the apical events of death receptor signaling." J Biol Chem 278(45): 44338-47.

Heinrich, M. C., C. L. Corless, G. D. Demetri, C. D. Blanke, M. von Mehren, H. Joensuu, L. S. McGreevey, C. J. Chen, A. D. Van den Abbeele, B. J. Druker, B. Kiese, B. Eisenberg, P. J. Roberts, S. Singer, C. D. Fletcher, S. Silberman, S. Dimitrijevic and J. A. Fletcher (2003). "Kinase mutations and imatinib response in patients with metastatic gastrointestinal stromal tumor." J Clin Oncol 21(23): 4342-9.

Hinds, M. G., R. S. Norton, D. L. Vaux and C. L. Day (1999). "Solution structure of a baculoviral inhibitor of apoptosis (IAP) repeat." Nat Struct Biol 6(7): 648-51.

Hinz, S., A. Trauzold, L. Boenicke, C. Sandberg, S. Beckmann, E. Bayer, H. Walczak, H. Kalthoff and H. Ungefroren (2000). "Bcl-XL protects pancreatic adenocarcinoma cells against CD95- and TRAIL-receptor-mediated apoptosis." Oncogene 19(48): 5477-86.

Hirte, H. W., D. A. Clark, J. Mazurka, G. O'Connell and J. Rusthoven (1992). "A rapid and simple method for the purification of tumor cells from ascitic fluid of ovarian carcinoma." Gynecol Oncol 44(3): 223-6.

Hitomi, J., D. E. Christofferson, A. Ng, J. Yao, A. Degterev, R. J. Xavier and J. Yuan (2008). "Identification of a molecular signaling network that regulates a cellular necrotic cell death pathway." Cell 135(7): 1311-23.

Hopkins-Donaldson, S., J. L. Bodmer, K. B. Bourloud, C. B. Brognara, J. Tschopp and N. Gross (2000). "Loss of caspase-8 expression in highly malignant human neuroblastoma cells correlates with resistance to tumor necrosis factor-related apoptosis-inducing ligand-induced apoptosis." Cancer Res 60(16): 4315-9.

Horak, P., D. Pils, A. Kaider, A. Pinter, K. Elandt, C. Sax, C. C. Zielinski, R. Horvat, R. Zeillinger, A. Reinthaller and M. Krainer (2005). "Perturbation of the tumor necrosis factor--related apoptosis-inducing ligand cascade in ovarian cancer: overexpression of FLIPL and deregulation of the functional receptors DR4 and DR5." Clin Cancer Res 11(24 Pt 1): 8585-91. 
Hornstein, M., M. J. Hoffmann, A. Alexa, M. Yamanaka, M. Muller, V. Jung, J. Rahnenfuhrer and W. A. Schulz (2008). "Protein phosphatase and TRAIL receptor genes as new candidate tumor genes on chromosome $8 \mathrm{p}$ in prostate cancer." Cancer Genomics Proteomics 5(2): 123-36.

Hsu, H., J. Xiong and D. V. Goeddel (1995). "The TNF receptor 1-associated protein TRADD signals cell death and NF-kappa B activation." Cell 81(4): 495-504.

Hsu, H., J. Huang, H. B. Shu, V. Baichwal and D. V. Goeddel (1996). "TNF-dependent recruitment of the protein kinase RIP to the TNF receptor-1 signaling complex." Immunity 4(4): 387-96.

Hu, L., C. Zaloudek, G. B. Mills, J. Gray and R. B. Jaffe (2000). "In vivo and in vitro ovarian carcinoma growth inhibition by a phosphatidylinositol 3-kinase inhibitor (LY294002)." Clin Cancer Res 6(3): 880-6.

Huang, Y., Y. C. Park, R. L. Rich, D. Segal, D. G. Myszka and H. Wu (2001). "Structural basis of caspase inhibition by XIAP: differential roles of the linker versus the BIR domain." Cell 104(5): 781-90.

Hunter, A. M., E. C. LaCasse and R. G. Korneluk (2007). "The inhibitors of apoptosis (IAPs) as cancer targets." Apoptosis 12(9): 1543-68.

Husain, A., G. He, E. S. Venkatraman and D. R. Spriggs (1998). "BRCA1 up-regulation is associated with repair-mediated resistance to cis-diamminedichloroplatinum(II)." Cancer Res 58(6): 1120-3.

Hymowitz, S. G., M. P. O'Connell, M. H. Ultsch, A. Hurst, K. Totpal, A. Ashkenazi, A. M. de Vos and R. F. Kelley (2000). "A unique zinc-binding site revealed by a highresolution X-ray structure of homotrimeric Apo2L/TRAIL." Biochemistry 39(4): 633 40.

Irmler, M., M. Thome, M. Hahne, P. Schneider, K. Hofmann, V. Steiner, J. L. Bodmer, M. Schroter, K. Burns, C. Mattmann, D. Rimoldi, L. E. French and J. Tschopp (1997). "Inhibition of death receptor signals by cellular FLIP." Nature 388(6638): 190-5.

Ishimura, N., H. Isomoto, S. F. Bronk and G. J. Gores (2006). "Trail induces cell migration and invasion in apoptosis-resistant cholangiocarcinoma cells." Am J Physiol Gastrointest Liver Physiol 290(1): G129-36.

Itamochi, H., J. Kigawa, T. Sugiyama, Y. Kikuchi, M. Suzuki and N. Terakawa (2002). "Low proliferation activity may be associated with chemoresistance in clear cell carcinoma of the ovary." Obstet Gynecol 100(2): 281-7.

Jin, Z. and W. S. El-Deiry (2006). "Distinct signaling pathways in TRAIL- versus tumor necrosis factor-induced apoptosis." Mol Cell Biol 26(21): 8136-48.

Jo, M., T. H. Kim, D. W. Seol, J. E. Esplen, K. Dorko, T. R. Billiar and S. C. Strom (2000). "Apoptosis induced in normal human hepatocytes by tumor necrosis factor-related apoptosis-inducing ligand." Nat Med 6(5): 564-7.

Jonsson, G., S. Paulie and A. Grandien (2003). "High level of cFLIP correlates with resistance to death receptor-induced apoptosis in bladder carcinoma cells." Anticancer Res 23(2B): 1213-8.

Jost, P. J., S. Grabow, D. Gray, M. D. McKenzie, U. Nachbur, D. C. Huang, P. Bouillet, H. E. Thomas, C. Borner, J. Silke, A. Strasser and T. Kaufmann (2009). "XIAP discriminates between type I and type II FAS-induced apoptosis." Nature 460(7258): 1035-9.

Kang, Y. C., K. M. Kim, K. S. Lee, S. Namkoong, S. J. Lee, J. A. Han, D. Jeoung, K. S. Ha, Y. G. Kwon and Y. M. Kim (2004). "Serum bioactive lysophospholipids prevent TRAIL-induced apoptosis via PI3K/Akt-dependent cFLIP expression and Bad phosphorylation." Cell Death Differ 11(12): 1287-98. 
Kielhorn, E., K. Schofield and D. L. Rimm (2002). "Use of magnetic enrichment for detection of carcinoma cells in fluid specimens." Cancer 94(1): 205-11.

Kim, S., J. Kang, J. Qiao, R. P. Thomas, B. M. Evers and D. H. Chung (2004). "Phosphatidylinositol 3-kinase inhibition down-regulates survivin and facilitates TRAIL-mediated apoptosis in neuroblastomas." J Pediatr Surg 39(4): 516-21.

Kimberley, F. C. and G. R. Screaton (2004). "Following a TRAIL: update on a ligand and its five receptors." Cell Res 14(5): 359-72.

Kischkel, F. C., D. A. Lawrence, A. Chuntharapai, P. Schow, K. J. Kim and A. Ashkenazi (2000). "Apo2L/TRAIL-dependent recruitment of endogenous FADD and caspase-8 to death receptors 4 and 5." Immunity 12(6): 611-20.

Kischkel, F. C., D. A. Lawrence, A. Tinel, H. LeBlanc, A. Virmani, P. Schow, A. Gazdar, J. Blenis, D. Arnott and A. Ashkenazi (2001). "Death receptor recruitment of endogenous caspase-10 and apoptosis initiation in the absence of caspase- 8 ." J Biol Chem 276(49): 46639-46.

Klopp, A. H., L. Lacerda, A. Gupta, B. G. Debeb, T. Solley, L. Li, E. Spaeth, W. Xu, X. Zhang, M. T. Lewis, J. M. Reuben, S. Krishnamurthy, M. Ferrari, R. Gaspar, T. A. Buchholz, M. Cristofanilli, F. Marini, M. Andreeff and W. A. Woodward (2010). "Mesenchymal stem cells promote mammosphere formation and decrease E-cadherin in normal and malignant breast cells." PLoS One 5(8).

Kobel, M., S. E. Kalloger, N. Boyd, S. McKinney, E. Mehl, C. Palmer, S. Leung, N. J. Bowen, D. N. Ionescu, A. Rajput, L. M. Prentice, D. Miller, J. Santos, K. Swenerton, C. B. Gilks and D. Huntsman (2008). "Ovarian carcinoma subtypes are different diseases: implications for biomarker studies." PLoS Med 5(12): e232.

Koschny, R., T. M. Ganten, J. Sykora, T. L. Haas, M. R. Sprick, A. Kolb, W. Stremmel and H. Walczak (2007a). "TRAIL/bortezomib cotreatment is potentially hepatotoxic but induces cancer-specific apoptosis within a therapeutic window." Hepatology 45(3): 649-58.

Koschny, R., H. Walczak and T. M. Ganten (2007b). "The promise of TRAIL--potential and risks of a novel anticancer therapy." J Mol Med 85(9): 923-35.

Koschny, R., H. Holland, J. Sykora, T. L. Haas, M. R. Sprick, T. M. Ganten, W. Krupp, M. Bauer, P. Ahnert, J. Meixensberger and H. Walczak (2007c). "Bortezomib sensitizes primary human astrocytoma cells of WHO grades I to IV for tumor necrosis factorrelated apoptosis-inducing ligand-induced apoptosis." Clin Cancer Res 13(11): 340312.

Koschny, R., H. Holland, J. Sykora, H. Erdal, W. Krupp, M. Bauer, U. Bockmuehl, P. Ahnert, J. Meixensberger, W. Stremmel, H. Walczak and T. M. Ganten (2010). "Bortezomib sensitizes primary human esthesioneuroblastoma cells to TRAIL-induced apoptosis." $\underline{\mathrm{J}}$ Neurooncol 97(2): 171-85.

Krueger, A., I. Schmitz, S. Baumann, P. H. Krammer and S. Kirchhoff (2001). "Cellular FLICE-inhibitory protein splice variants inhibit different steps of caspase-8 activation at the CD95 death-inducing signaling complex." J Biol Chem 276(23): 20633-40.

Kuo, K. T., T. L. Mao, S. Jones, E. Veras, A. Ayhan, T. L. Wang, R. Glas, D. Slamon, V. E. Velculescu, R. J. Kuman and M. Shih Ie (2009). "Frequent activating mutations of PIK3CA in ovarian clear cell carcinoma." Am J Pathol 174(5): 1597-601.

Kuwabara, Y., T. Yamada, K. Yamazaki, W. L. Du, K. Banno, D. Aoki and M. Sakamoto (2008). "Establishment of an ovarian metastasis model and possible involvement of Ecadherin down-regulation in the metastasis." Cancer Sci 99(10): 1933-9.

LaCasse, E. C., D. J. Mahoney, H. H. Cheung, S. Plenchette, S. Baird and R. G. Korneluk (2008). "IAP-targeted therapies for cancer." Oncogene 27(48): 6252-75. 
Lamhamedi-Cherradi, S. E., S. J. Zheng, K. A. Maguschak, J. Peschon and Y. H. Chen (2003). "Defective thymocyte apoptosis and accelerated autoimmune diseases in TRAIL-/- mice." Nat Immunol 4(3): 255-60.

Langdon, S. P., S. S. Lawrie, F. G. Hay, M. M. Hawkes, A. McDonald, I. P. Hayward, D. J. Schol, J. Hilgers, R. C. Leonard and J. F. Smyth (1988). "Characterization and properties of nine human ovarian adenocarcinoma cell lines." Cancer Res 48(21): 6166-72.

Latza, U., G. Niedobitek, R. Schwarting, H. Nekarda and H. Stein (1990). "Ber-EP4: new monoclonal antibody which distinguishes epithelia from mesothelial." J Clin Pathol 43(3): 213-9.

LeBlanc, H. N. and A. Ashkenazi (2003). "Apo2L/TRAIL and its death and decoy receptors." Cell Death Differ 10(1): 66-75.

Lecis, D., C. Drago, L. Manzoni, P. Seneci, C. Scolastico, E. Mastrangelo, M. Bolognesi, A. Anichini, H. Kashkar, H. Walczak and D. Delia (2010). "Novel SMAC-mimetics synergistically stimulate melanoma cell death in combination with TRAIL and Bortezomib." Br J Cancer 102(12): 1707-16.

Lee, Y. H., A. R. Albig, M. Regner, B. J. Schiemann and W. P. Schiemann (2008). "Fibulin-5 initiates epithelial-mesenchymal transition (EMT) and enhances EMT induced by TGF-beta in mammary epithelial cells via a MMP-dependent mechanism." Carcinogenesis 29(12): 2243-51.

Lessene, G., P. E. Czabotar and P. M. Colman (2008). "BCL-2 family antagonists for cancer therapy." Nat Rev Drug Discov 7(12): 989-1000.

Leverkus, M., M. Neumann, T. Mengling, C. T. Rauch, E. B. Brocker, P. H. Krammer and H. Walczak (2000). "Regulation of tumor necrosis factor-related apoptosis-inducing ligand sensitivity in primary and transformed human keratinocytes." Cancer Res 60(3): 553-9.

Leverkus, M., M. R. Sprick, T. Wachter, T. Mengling, B. Baumann, E. Serfling, E. B. Brocker, M. Goebeler, M. Neumann and H. Walczak (2003). "Proteasome inhibition results in TRAIL sensitization of primary keratinocytes by removing the resistancemediating block of effector caspase maturation." Mol Cell Biol 23(3): 777-90.

Li, H., H. Zhu, C. J. Xu and J. Yuan (1998). "Cleavage of BID by caspase 8 mediates the mitochondrial damage in the Fas pathway of apoptosis." Cell 94(4): 491-501.

Li, J., H. Sasaki, Y. L. Sheng, D. Schneiderman, C. W. Xiao, F. Kotsuji and B. K. Tsang (2000). "Apoptosis and chemoresistance in human ovarian cancer: is Xiap a determinant?" Biol Signals Recept 9(2): 122-30.

Li, L., R. M. Thomas, H. Suzuki, J. K. De Brabander, X. Wang and P. G. Harran (2004). "A small molecule Smac mimic potentiates TRAIL- and TNFalpha-mediated cell death." Science 305(5689): 1471-4.

Lin, Y., A. Devin, A. Cook, M. M. Keane, M. Kelliher, S. Lipkowitz and Z. G. Liu (2000). "The death domain kinase RIP is essential for TRAIL (Apo2L)-induced activation of IkappaB kinase and c-Jun N-terminal kinase." Mol Cell Biol 20(18): 6638-45.

Litvinov, S. V., M. P. Velders, H. A. Bakker, G. J. Fleuren and S. O. Warnaar (1994). "EpCAM: a human epithelial antigen is a homophilic cell-cell adhesion molecule." J Cell Biol 125(2): 437-46.

Litvinov, S. V., M. Balzar, M. J. Winter, H. A. Bakker, I. H. Briaire-de Bruijn, F. Prins, G. J. Fleuren and S. O. Warnaar (1997). "Epithelial cell adhesion molecule (Ep-CAM) modulates cell-cell interactions mediated by classic cadherins." J Cell Biol 139(5): 1337-48.

Lu, J., L. Bai, H. Sun, Z. Nikolovska-Coleska, D. McEachern, S. Qiu, R. S. Miller, H. Yi, S. Shangary, Y. Sun, J. L. Meagher, J. A. Stuckey and S. Wang (2008). "SM-164: a 
novel, bivalent Smac mimetic that induces apoptosis and tumor regression by concurrent removal of the blockade of cIAP-1/2 and XIAP." Cancer Res 68(22): 938493.

MacFarlane, M. (2003). "TRAIL-induced signalling and apoptosis." Toxicol Lett 139(2-3): 89-97.

Maduro, J. H., E. G. de Vries, G. J. Meersma, B. M. Hougardy, A. G. van der Zee and S. de Jong (2008). "Targeting pro-apoptotic trail receptors sensitizes HeLa cervical cancer cells to irradiation-induced apoptosis." Int J Radiat Oncol Biol Phys 72(2): 543-52.

Maetzel, D., S. Denzel, B. Mack, M. Canis, P. Went, M. Benk, C. Kieu, P. Papior, P. A. Baeuerle, M. Munz and O. Gires (2009). "Nuclear signalling by tumour-associated antigen EpCAM." Nat Cell Biol 11(2): 162-71.

Mariani, S. M. and P. H. Krammer (1998). "Differential regulation of TRAIL and CD95 ligand in transformed cells of the T and B lymphocyte lineage." Eur J Immunol 28(3): 973-82.

Martelli, A. M., P. L. Tazzari, G. Tabellini, R. Bortul, A. M. Billi, L. Manzoli, A. Ruggeri, R. Conte and L. Cocco (2003). "A new selective AKT pharmacological inhibitor reduces resistance to chemotherapeutic drugs, TRAIL, all-trans-retinoic acid, and ionizing radiation of human leukemia cells." Leukemia 17(9): 1794-805.

Martin, D. A., R. M. Siegel, L. Zheng and M. J. Lenardo (1998). "Membrane oligomerization and cleavage activates the caspase-8 (FLICE/MACHalpha1) death signal." J Biol Chem 273(8): 4345-9.

McKenzie, M., D. Liolitsa and M. G. Hanna (2004). "Mitochondrial disease: Mutations and mechanisms." Neurochem Res 29(3): 589-600.

Merino, D., N. Lalaoui, A. Morizot, P. Schneider, E. Solary and O. Micheau (2006). "Differential inhibition of TRAIL-mediated DR5-DISC formation by decoy receptors 1 and 2." Mol Cell Biol 26(19): 7046-55.

Micheau, O. and D. Merino (2004). "Controlling TRAIL-mediated caspase-3 activation." Leukemia 18(10): 1578-80.

Micheau, O., M. Thome, P. Schneider, N. Holler, J. Tschopp, D. W. Nicholson, C. Briand and M. G. Grutter (2002). "The long form of FLIP is an activator of caspase- 8 at the Fas death-inducing signaling complex." J Biol Chem 277(47): 45162-71.

Morgillo, F., E. D'Aiuto, T. Troiani, E. Martinelli, T. Cascone, R. De Palma, M. Orditura, F. De Vita and F. Ciardiello (2010). "Antitumor activity of bortezomib in human cancer cells with acquired resistance to anti-epidermal growth factor receptor tyrosine kinase inhibitors." Lung Cancer. (Article in press)

Munz, M., C. Kieu, B. Mack, B. Schmitt, R. Zeidler and O. Gires (2004). "The carcinomaassociated antigen EpCAM upregulates c-myc and induces cell proliferation." Oncogene 23(34): 5748-58.

Muzio, M., B. R. Stockwell, H. R. Stennicke, G. S. Salvesen and V. M. Dixit (1998). "An induced proximity model for caspase-8 activation." J Biol Chem 273(5): 2926-30.

Nagata, S. (2000). "Apoptotic DNA fragmentation." Exp Cell Res 256(1): 12-8.

Nakayama, K., N. Nakayama, R. J. Kurman, L. Cope, G. Pohl, Y. Samuels, V. E. Velculescu, T. L. Wang and M. Shih Ie (2006). "Sequence mutations and amplification of PIK3CA and AKT2 genes in purified ovarian serous neoplasms." Cancer Biol Ther 5(7): 77985.

Nicoletti, I., G. Migliorati, M. C. Pagliacci, F. Grignani and C. Riccardi (1991). "A rapid and simple method for measuring thymocyte apoptosis by propidium iodide staining and flow cytometry." J Immunol Methods 139(2): 271-9.

Nicotera, P., M. Leist and L. Manzo (1999). "Neuronal cell death: a demise with different shapes." Trends Pharmacol Sci 20(2): 46-51. 
O'Brien, S. G., F. Guilhot, R. A. Larson, I. Gathmann, M. Baccarani, F. Cervantes, J. J. Cornelissen, T. Fischer, A. Hochhaus, T. Hughes, K. Lechner, J. L. Nielsen, P. Rousselot, J. Reiffers, G. Saglio, J. Shepherd, B. Simonsson, A. Gratwohl, J. M. Goldman, H. Kantarjian, K. Taylor, G. Verhoef, A. E. Bolton, R. Capdeville and B. J. Druker (2003). "Imatinib compared with interferon and low-dose cytarabine for newly diagnosed chronic-phase chronic myeloid leukemia." N Engl J Med 348(11): 9941004.

Osta, W. A., Y. Chen, K. Mikhitarian, M. Mitas, M. Salem, Y. A. Hannun, D. J. Cole and W. E. Gillanders (2004). "EpCAM is overexpressed in breast cancer and is a potential target for breast cancer gene therapy." Cancer Res 64(16): 5818-24.

Ozoren, N. and W. El-Deiry (2002). "Heat shock protects HCT116 and H460 cells from TRAIL-induced apoptosis." Exp Cell Res 281(2): 175-81.

Pan, G., K. O'Rourke, A. M. Chinnaiyan, R. Gentz, R. Ebner, J. Ni and V. M. Dixit (1997). "The receptor for the cytotoxic ligand TRAIL." Science 276(5309): 111-3.

Pan, G., J. Ni, G. Yu, Y. F. Wei and V. M. Dixit (1998). "TRUNDD, a new member of the TRAIL receptor family that antagonizes TRAIL signalling." FEBS Lett 424(1-2): 415.

Panka, D. J., T. Mano, T. Suhara, K. Walsh and J. W. Mier (2001). "Phosphatidylinositol 3kinase/Akt activity regulates c-FLIP expression in tumor cells." J Biol Chem 276(10): 6893-6.

Pasquini, L., A. Petronelli, E. Petrucci, E. Saulle, G. Mariani, G. Scambia, P. BenedettiPanici, S. Greggi, F. Cognetti and U. Testa (2010). "Primary ovarian cancer cells are sensitive to the proaptotic effects of proteasome inhibitors." Int J Oncol 36(3): 707-13.

Prasad, K. V. and B. S. Prabhakar (2003). "Apoptosis and autoimmune disorders." Autoimmunity 36(6-7): 323-30.

Probst, B. L., L. Liu, V. Ramesh, L. Li, H. Sun, J. D. Minna and L. Wang (2010). "Smac mimetics increase cancer cell response to chemotherapeutics in a TNF-alphadependent manner." Cell Death Differ 17(10): 1645-54.

Quinn, J. E., R. D. Kennedy, P. B. Mullan, P. M. Gilmore, M. Carty, P. G. Johnston and D. P. Harkin (2003). "BRCA1 functions as a differential modulator of chemotherapyinduced apoptosis." Cancer Res 63(19): 6221-8.

Renatus, M., H. R. Stennicke, F. L. Scott, R. C. Liddington and G. S. Salvesen (2001). "Dimer formation drives the activation of the cell death protease caspase 9." Proc Natl Acad Sci U S A 98(25): 14250-5.

Rheaume, E., L. Y. Cohen, F. Uhlmann, C. Lazure, A. Alam, J. Hurwitz, R. P. Sekaly and F. Denis (1997). "The large subunit of replication factor C is a substrate for caspase-3 in vitro and is cleaved by a caspase-3-like protease during Fas-mediated apoptosis." EMBO J 16(21): 6346-54.

Ricci, M. S., S. H. Kim, K. Ogi, J. P. Plastaras, J. Ling, W. Wang, Z. Jin, Y. Y. Liu, D. T. Dicker, P. J. Chiao, K. T. Flaherty, C. D. Smith and W. S. El-Deiry (2007).

"Reduction of TRAIL-induced Mcl-1 and cIAP2 by c-Myc or sorafenib sensitizes resistant human cancer cells to TRAIL-induced death." Cancer Cell 12(1): 66-80.

Riedl, S. J. and G. S. Salvesen (2007). "The apoptosome: signalling platform of cell death." Nat Rev Mol Cell Biol 8(5): 405-13.

Rothe, M., V. Sarma, V. M. Dixit and D. V. Goeddel (1995). "TRAF2-mediated activation of NF-kappa B by TNF receptor 2 and CD40." Science 269(5229): 1424-7.

Samuels, Y., Z. Wang, A. Bardelli, N. Silliman, J. Ptak, S. Szabo, H. Yan, A. Gazdar, S. M. Powell, G. J. Riggins, J. K. Willson, S. Markowitz, K. W. Kinzler, B. Vogelstein and V. E. Velculescu (2004). "High frequency of mutations of the PIK3CA gene in human cancers." Science 304(5670): 554. 
Samuels, Y., L. A. Diaz, Jr., O. Schmidt-Kittler, J. M. Cummins, L. Delong, I. Cheong, C. Rago, D. L. Huso, C. Lengauer, K. W. Kinzler, B. Vogelstein and V. E. Velculescu (2005). "Mutant PIK3CA promotes cell growth and invasion of human cancer cells." Cancer Cell 7(6): 561-73.

Sayers, T. J., A. D. Brooks, C. Y. Koh, W. Ma, N. Seki, A. Raziuddin, B. R. Blazar, X. Zhang, P. J. Elliott and W. J. Murphy (2003). "The proteasome inhibitor PS-341 sensitizes neoplastic cells to TRAIL-mediated apoptosis by reducing levels of cFLIP." Blood 102(1): 303-10.

Scaffidi, C., S. Fulda, A. Srinivasan, C. Friesen, F. Li, K. J. Tomaselli, K. M. Debatin, P. H. Krammer and M. E. Peter (1998). "Two CD95 (APO-1/Fas) signaling pathways." EMBO J 17(6): 1675-87.

Scaffidi, C., I. Schmitz, P. H. Krammer and M. E. Peter (1999). "The role of c-FLIP in modulation of CD95-induced apoptosis." J Biol Chem 274(3): 1541-8.

Schneider, P., D. Olson, A. Tardivel, B. Browning, A. Lugovskoy, D. Gong, M. Dobles, S. Hertig, K. Hofmann, H. Van Vlijmen, Y. M. Hsu, L. C. Burkly, J. Tschopp and T. S. Zheng (2003). "Identification of a new murine tumor necrosis factor receptor locus that contains two novel murine receptors for tumor necrosis factor-related apoptosisinducing ligand (TRAIL)." J Biol Chem 278(7): 5444-54.

Screaton, G. R., J. Mongkolsapaya, X. N. Xu, A. E. Cowper, A. J. McMichael and J. I. Bell (1997). "TRICK2, a new alternatively spliced receptor that transduces the cytotoxic signal from TRAIL." Curr Biol 7(9): 693-6.

Sheikh, M. S., Y. Huang, E. A. Fernandez-Salas, W. S. El-Deiry, H. Friess, S. Amundson, J. Yin, S. J. Meltzer, N. J. Holbrook and A. J. Fornace, Jr. (1999). "The antiapoptotic decoy receptor TRID/TRAIL-R3 is a p53-regulated DNA damage-inducible gene that is overexpressed in primary tumors of the gastrointestinal tract." Oncogene 18(28): 4153-9.

Shepherd, T. G., B. L. Theriault, E. J. Campbell and M. W. Nachtigal (2006). "Primary culture of ovarian surface epithelial cells and ascites-derived ovarian cancer cells from patients." Nature Protocols 1(6): 2643-2649.

Shi, Y. (2004). "Caspase activation, inhibition, and reactivation: a mechanistic view." Protein Sci 13(8): 1979-87.

Shu, H. B., M. Takeuchi and D. V. Goeddel (1996). "The tumor necrosis factor receptor 2 signal transducers TRAF2 and c-IAP1 are components of the tumor necrosis factor receptor 1 signaling complex." Proc Natl Acad Sci U S A 93(24): 13973-8.

Siegmund, D., P. Hadwiger, K. Pfizenmaier, H. P. Vornlocher and H. Wajant (2002). "Selective inhibition of FLICE-like inhibitory protein expression with small interfering RNA oligonucleotides is sufficient to sensitize tumor cells for TRAILinduced apoptosis." Mol Med 8(11): 725-32.

Song, J. J. and Y. J. Lee (2008). "Differential cleavage of Mst1 by caspase-7/-3 is responsible for TRAIL-induced activation of the MAPK superfamily." Cell Signal 20(5): 892-906.

Song, K., Y. Chen, R. Goke, A. Wilmen, C. Seidel, A. Goke and B. Hilliard (2000). "Tumor necrosis factor-related apoptosis-inducing ligand (TRAIL) is an inhibitor of autoimmune inflammation and cell cycle progression." J Exp Med 191(7): 1095-104.

Sprick, M. R., M. A. Weigand, E. Rieser, C. T. Rauch, P. Juo, J. Blenis, P. H. Krammer and H. Walczak (2000). "FADD/MORT1 and caspase- 8 are recruited to TRAIL receptors 1 and 2 and are essential for apoptosis mediated by TRAIL receptor 2." Immunity 12(6): 599-609.

Sprick, M. R., E. Rieser, H. Stahl, A. Grosse-Wilde, M. A. Weigand and H. Walczak (2002). "Caspase-10 is recruited to and activated at the native TRAIL and CD95 death- 
inducing signalling complexes in a FADD-dependent manner but can not functionally substitute caspase-8." EMBO J 21(17): 4520-30.

Sprick, M. R. and H. Walczak (2004). "The interplay between the Bcl-2 family and death receptor-mediated apoptosis." Biochim Biophys Acta 1644(2-3): 125-32.

Srinivasula, S. M., R. Hegde, A. Saleh, P. Datta, E. Shiozaki, J. Chai, R. A. Lee, P. D. Robbins, T. Fernandes-Alnemri, Y. Shi and E. S. Alnemri (2001). "A conserved XIAP-interaction motif in caspase- 9 and Smac/DIABLO regulates caspase activity and apoptosis." Nature 410(6824): 112-6.

Stennicke, H. R., Q. L. Deveraux, E. W. Humke, J. C. Reed, V. M. Dixit and G. S. Salvesen (1999). "Caspase-9 can be activated without proteolytic processing." J Biol Chem 274(13): 8359-62.

Strasser, A. (2005). "The role of BH3-only proteins in the immune system." Nat Rev Immunol 5(3): 189-200.

Sugiyama, T., T. Kamura, J. Kigawa, N. Terakawa, Y. Kikuchi, T. Kita, M. Suzuki, I. Sato and K. Taguchi (2000). "Clinical characteristics of clear cell carcinoma of the ovary: a distinct histologic type with poor prognosis and resistance to platinum-based chemotherapy." Cancer 88(11): 2584-9.

Sun, C., M. Cai, A. H. Gunasekera, R. P. Meadows, H. Wang, J. Chen, H. Zhang, W. Wu, N. $\mathrm{Xu}, \mathrm{S}$. C. Ng and S. W. Fesik (1999). "NMR structure and mutagenesis of the inhibitor-of-apoptosis protein XIAP." Nature 401(6755): 818-22.

Swisher, E. M., R. M. Gonzalez, T. Taniguchi, R. L. Garcia, T. Walsh, B. A. Goff and P. Welcsh (2009). "Methylation and protein expression of DNA repair genes: association with chemotherapy exposure and survival in sporadic ovarian and peritoneal carcinomas." Mol Cancer 8: 48.

Tang, C., Y. H. Lu, J. H. Xie, F. Wang, J. N. Zou, J. S. Yang, Y. Y. Xing and T. Xi (2009). "Downregulation of survivin and activation of caspase-3 through the PI3K/Akt pathway in ursolic acid-induced HepG2 cell apoptosis." Anticancer Drugs 20(4): 24958.

Taniai, M., A. Grambihler, H. Higuchi, N. Werneburg, S. F. Bronk, D. J. Farrugia, S. H. Kaufmann and G. J. Gores (2004). "Mcl-1 mediates tumor necrosis factor-related apoptosis-inducing ligand resistance in human cholangiocarcinoma cells." $\underline{\text { Cancer Res }}$ 64(10): 3517-24.

Tazzari, P. L., G. Tabellini, F. Ricci, V. Papa, R. Bortul, F. Chiarini, C. Evangelisti, G. Martinelli, A. Bontadini, L. Cocco, J. A. McCubrey and A. M. Martelli (2008). "Synergistic proapoptotic activity of recombinant TRAIL plus the Akt inhibitor Perifosine in acute myelogenous leukemia cells." Cancer Res 68(22): 9394-403.

Thrall, M., H. H. Gallion, R. Kryscio, M. Kapali, D. K. Armstrong and J. A. DeLoia (2006). "BRCA1 expression in a large series of sporadic ovarian carcinomas: a Gynecologic Oncology Group study." Int J Gynecol Cancer 16 Suppl 1: 166-71.

Tomek, S., P. Horak, I. Pribill, G. Haller, M. Rossler, C. C. Zielinski, D. Pils and M. Krainer (2004). "Resistance to TRAIL-induced apoptosis in ovarian cancer cell lines is overcome by co-treatment with cytotoxic drugs." Gynecol Oncol 94(1): 107-14.

Towbin, H., T. Staehelin and J. Gordon (1979). "Electrophoretic transfer of proteins from polyacrylamide gels to nitrocellulose sheets: procedure and some applications." Proc Natl Acad Sci U S A 76(9): 4350-4.

Trauzold, A., D. Siegmund, B. Schniewind, B. Sipos, J. Egberts, D. Zorenkov, D. Emme, C. Roder, H. Kalthoff and H. Wajant (2006). "TRAIL promotes metastasis of human pancreatic ductal adenocarcinoma." Oncogene 25(56): 7434-9. 
Tsao, D. H., T. McDonagh, J. B. Telliez, S. Hsu, K. Malakian, G. Y. Xu and L. L. Lin (2000). "Solution structure of N-TRADD and characterization of the interaction of N-TRADD and C-TRAF2, a key step in the TNFR1 signaling pathway." Mol Cell 5(6): 1051-7.

Varfolomeev, E., H. Maecker, D. Sharp, D. Lawrence, M. Renz, D. Vucic and A. Ashkenazi (2005). "Molecular determinants of kinase pathway activation by Apo2 ligand/tumor necrosis factor-related apoptosis-inducing ligand." J Biol Chem 280(49): 40599-608.

Varfolomeev, E., J. W. Blankenship, S. M. Wayson, A. V. Fedorova, N. Kayagaki, P. Garg, K. Zobel, J. N. Dynek, L. O. Elliott, H. J. Wallweber, J. A. Flygare, W. J. Fairbrother, K. Deshayes, V. M. Dixit and D. Vucic (2007). "IAP antagonists induce autoubiquitination of c-IAPs, NF-kappaB activation, and TNFalpha-dependent apoptosis." Cell 131(4): 669-81.

Varfolomeev, E., T. Goncharov, A. V. Fedorova, J. N. Dynek, K. Zobel, K. Deshayes, W. J. Fairbrother and D. Vucic (2008). "c-IAP1 and c-IAP2 are critical mediators of tumor necrosis factor alpha (TNFalpha)-induced NF-kappaB activation." J Biol Chem 283(36): 24295-9.

Verhagen, A. M., P. G. Ekert, M. Pakusch, J. Silke, L. M. Connolly, G. E. Reid, R. L. Moritz, R. J. Simpson and D. L. Vaux (2000). "Identification of DIABLO, a mammalian protein that promotes apoptosis by binding to and antagonizing IAP proteins." Cell 102(1): 43-53.

Vince, J. E., W. W. Wong, N. Khan, R. Feltham, D. Chau, A. U. Ahmed, C. A. Benetatos, S. K. Chunduru, S. M. Condon, M. McKinlay, R. Brink, M. Leverkus, V. Tergaonkar, P. Schneider, B. A. Callus, F. Koentgen, D. L. Vaux and J. Silke (2007). "IAP antagonists target cIAP1 to induce TNFalpha-dependent apoptosis." Cell 131(4): 68293.

Visvader, J. E. and G. J. Lindeman (2008). "Cancer stem cells in solid tumours: accumulating evidence and unresolved questions." Nat Rev Cancer 8(10): 755-68.

Vogler, M., K. Durr, M. Jovanovic, K. M. Debatin and S. Fulda (2007). "Regulation of TRAIL-induced apoptosis by XIAP in pancreatic carcinoma cells." Oncogene 26(2): 248-57.

Vogler, M., H. Walczak, D. Stadel, T. L. Haas, F. Genze, M. Jovanovic, U. Bhanot, C. Hasel, P. Moller, J. E. Gschwend, T. Simmet, K. M. Debatin and S. Fulda (2009). "Small molecule XIAP inhibitors enhance TRAIL-induced apoptosis and antitumor activity in preclinical models of pancreatic carcinoma." Cancer Res 69(6): 2425-34.

Wajant, H., D. Moosmayer, T. Wuest, T. Bartke, E. Gerlach, U. Schonherr, N. Peters, P. Scheurich and K. Pfizenmaier (2001). "Differential activation of TRAIL-R1 and -2 by soluble and membrane TRAIL allows selective surface antigen-directed activation of TRAIL-R2 by a soluble TRAIL derivative." Oncogene 20(30): 4101-6.

Walczak, H., M. A. Degli-Esposti, R. S. Johnson, P. J. Smolak, J. Y. Waugh, N. Boiani, M. S. Timour, M. J. Gerhart, K. A. Schooley, C. A. Smith, R. G. Goodwin and C. T. Rauch (1997). "TRAIL-R2: a novel apoptosis-mediating receptor for TRAIL." EMBO J 16(17): 5386-97.

Walczak, H., R. E. Miller, K. Ariail, B. Gliniak, T. S. Griffith, M. Kubin, W. Chin, J. Jones, A. Woodward, T. Le, C. Smith, P. Smolak, R. G. Goodwin, C. T. Rauch, J. C. Schuh and D. H. Lynch (1999). "Tumoricidal activity of tumor necrosis factor-related apoptosis-inducing ligand in vivo." Nat Med 5(2): 157-63.

Walczak, H. and T. L. Haas (2008). "Biochemical analysis of the native TRAIL deathinducing signaling complex." Methods Mol Biol 414: 221-39. 
Wang, J., H. J. Chun, W. Wong, D. M. Spencer and M. J. Lenardo (2001). "Caspase-10 is an initiator caspase in death receptor signaling." Proc Natl Acad Sci U S A 98(24): 13884-8.

Waterhouse, N. J., J. E. Ricci and D. R. Green (2002). "And all of a sudden it's over: mitochondrial outer-membrane permeabilization in apoptosis." Biochimie 84(2-3): 113-21.

Went, P. T., A. Lugli, S. Meier, M. Bundi, M. Mirlacher, G. Sauter and S. Dirnhofer (2004). "Frequent EpCam protein expression in human carcinomas." Hum Pathol 35(1): 1228.

Wiley, S. R., K. Schooley, P. J. Smolak, W. S. Din, C. P. Huang, J. K. Nicholl, G. R. Sutherland, T. D. Smith, C. Rauch, C. A. Smith and et al. (1995). "Identification and characterization of a new member of the TNF family that induces apoptosis." Immunity 3(6): 673-82.

Willis, S. N., J. I. Fletcher, T. Kaufmann, M. F. van Delft, L. Chen, P. E. Czabotar, H. Ierino, E. F. Lee, W. D. Fairlie, P. Bouillet, A. Strasser, R. M. Kluck, J. M. Adams and D. C. Huang (2007). "Apoptosis initiated when BH3 ligands engage multiple Bcl-2 homologs, not Bax or Bak." Science 315(5813): 856-9.

Wissink, E. H., I. Verbrugge, S. R. Vink, M. B. Schader, U. Schaefer, H. Walczak, J. Borst and M. Verheij (2006). "TRAIL enhances efficacy of radiotherapy in a p53 mutant, Bcl-2 overexpressing lymphoid malignancy." Radiother Oncol 80(2): 214-22.

Wu, G. S., T. F. Burns, Y. Zhan, E. S. Alnemri and W. S. El-Deiry (1999). "Molecular cloning and functional analysis of the mouse homologue of the KILLER/DR5 tumor necrosis factor-related apoptosis-inducing ligand (TRAIL) death receptor." Cancer Res 59(12): 2770-5.

Wu, G. S., K. Kim and W. S. el-Deiry (2000). "KILLER/DR5, a novel DNA-damage inducible death receptor gene, links the p53-tumor suppressor to caspase activation and apoptotic death." Adv Exp Med Biol 465: 143-51.

Xu, J., J. Y. Zhou, W. Z. Wei and G. S. Wu (2010). "Activation of the Akt survival pathway contributes to TRAIL resistance in cancer cells." PLoS One 5(4): e10226.

Yap, T. A., C. P. Carden and S. B. Kaye (2009). "Beyond chemotherapy: targeted therapies in ovarian cancer." Nat Rev Cancer 9(3): 167-81.

Youle, R. J. and A. Strasser (2008). "The BCL-2 protein family: opposing activities that mediate cell death." Nat Rev Mol Cell Biol 9(1): 47-59.

Zhang, J., P. L. Yang and N. S. Gray (2009). "Targeting cancer with small molecule kinase inhibitors." Nat Rev Cancer 9(1): 28-39.

Zhang, X. M., H. Lin, C. Chen and B. D. Chen (1999). "Inhibition of ubiquitin-proteasome pathway activates a caspase-3-like protease and induces Bcl-2 cleavage in human M07e leukaemic cells." Biochem J 340 ( Pt 1): 127-33.

Zisman, A., C. P. Ng, A. J. Pantuck, B. Bonavida and A. S. Belldegrun (2001). "Actinomycin $\mathrm{D}$ and gemcitabine synergistically sensitize androgen-independent prostate cancer cells to Apo2L/TRAIL-mediated apoptosis." J Immunother 24(6): 459-71. 


\section{Acknowledgments:}

First and foremost I would like to thank my supervisor Professor Dr. Henning Walczak for giving me the opportunity to work on a great project in a fantastic research group and accepting the role as second referee for this thesis.

Furthermore, I would like to thank Professor Dr. Claudia Binder at the Division of Haematology/Oncology of the Georg-August-University Goettingen for her support and for accepting the role as a first referee for this thesis.

This thesis would not have been possible without the support of all the people in the group. I want to thank all of you for giving me such a nice time working in the lab. Above all I would like to thank Silvia Prieske for many scientific discussions and technical help throughout the whole year. Furthermore, I especially would like to thank Dr. Kerstin Papenfuss and Dr. Chahrazade Kantari for their manifold help, also concerning my thesis.

Moreover, I would like to thank Eva Rieser, Stefanie Cordier, Björn Gerlach, Anna Schmuckle, Dr. Christoph Emmerich, Frank Reichenbach for their technical advise and help. I would also like to thank Dr. Roshan Agarwal for his collaboration on the primary ovarian cancer purification and many helpful scientific discussions.

Furthermore, I would also like to show my gratitude to the Boehringer Ingelheim Fonds for providing me with a MD Fellowship that gave me excellent research conditions and allowed me to completely focus on my thesis without any financial worries. 